\title{
Article
}

\section{Does sexual selection explain human sex differences in aggression?}

\author{
Archer, John \\ Available at http://clok.uclan.ac.uk/1155/ \\ Archer, John ORCID: 0000-0003-0483-1576 (2009) Does sexual selection \\ explain human sex differences in aggression? Behavioral and Brain Sciences, \\ 32 (3-4). pp. 249-311. ISSN 0140-525X
}

It is advisable to refer to the publisher's version if you intend to cite from the work. http://dx.doi.org/10.1017/S0140525X09990951

For more information about UCLan's research in this area go to

http://www.uclan.ac.uk/researchgroups/ and search for <name of research Group>.

For information about Research generally at UCLan please go to http://www.uclan.ac.uk/research/

All outputs in CLoK are protected by Intellectual Property Rights law, including Copyright law. Copyright, IPR and Moral Rights for the works on this site are retained by the individual authors and/or other copyright owners. Terms and conditions for use of this material are defined in the policies page.

\section{CLoK}

Central Lancashire online Knowledge www.clok.uclan.ac.uk

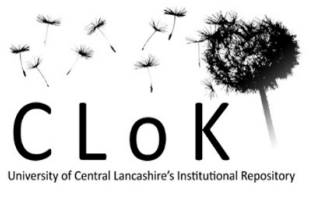




\title{
Does sexual selection explain human sex differences in aggression?
}

\author{
John Archer \\ School of Psychology, University of Central Lancashire, Preston PR1 2HE, \\ United Kingdom \\ jarcher@uclan.ac.uk \\ http://www.uclan.ac.uk/scitech/research/rae2008/psychology/ \\ staff_profiles/jarcher.php
}

\begin{abstract}
I argue that the magnitude and nature of sex differences in aggression, their development, causation, and variability, can be better explained by sexual selection than by the alternative biosocial version of social role theory. Thus, sex differences in physical aggression increase with the degree of risk, occur early in life, peak in young adulthood, and are likely to be mediated by greater male impulsiveness, and greater female fear of physical danger. Male variability in physical aggression is consistent with an alternative life history perspective, and context-dependent variability with responses to reproductive competition, although some variability follows the internal and external influences of social roles. Other sex differences, in variance in reproductive output, threat displays, size and strength, maturation rates, and mortality and conception rates, all indicate that male aggression is part of a sexually selected adaptive complex. Physical aggression between partners can be explained using different evolutionary principles, arising from the conflicts of interest between males and females entering a reproductive alliance, combined with variability following differences in societal gender roles. In this case, social roles are particularly important since they enable both the relatively equality in physical aggression between partners from Western nations, and the considerable cross-national variability, to be explained.
\end{abstract}

Keywords: aggression; partner violence; sex differences; sexual selection; social role theory

\section{Introduction}

Darwin (1859/1911; 1871/1901) regarded the greater proneness to physical aggression by men than women as part of a general mammalian pattern, which can be explained by one aspect of sexual selection, inter-male competition. Within the social sciences there is a long tradition of explaining sex differences in social behavior, including aggression, in human-centered terms, as a consequence of social influences. In this article, I argue that sexual selection provides a better explanation for human sex differences in aggression than the main contemporary social science approach, the social role theory.

Aggression is typically defined as behavior intended to harm another individual, and its study was originally restricted to direct physical and verbal forms. Psychologists began systematically documenting human sex differences in these types of aggression in the 1920s, and studies have continued to the present time. The forms of aggression now include indirect (or "relational") aggression, which Feshbach (1969) and Lagerspetz et al. (1988) found to be more typical of girls than boys. They suggested that the sex difference, hitherto characterized as involving "aggression," was more accurately viewed as involving its form. Contemporary studies include both direct and indirect aggression. A separate extensive literature documents sex differences (or similarities) in physical aggression between partners, although this is not usually considered in discussions of sex differences in aggression. I do consider the evolutionary and social forces underlying aggression between partners in this article, so as to provide a comprehensive explanation of sex differences in aggression. Nevertheless, most of the discussion is devoted to within-sex differences, the main concern of sexual selection and social role accounts. In assessing the evidence, I address the issues of ultimate origins, development, mediating variables, and sources of individual differences, in relation to predictions derived from the two theories. I argue that sexual selection provides the more complete explanation of the origins of sex differences in aggression, and that these differences are linked to a range of other features indicting the operation of sexual selection in humans. Although reformulations of social role theory do encompass some evolved differences, they are restricted to physical attributes, which form only part of the sexual selection view.

John ARCher, Professor of Psychology at the University of Central Lancashire, Preston, United Kingdom, is the author of more than 100 articles, in a wide range of journals, in the areas of animal aggression and emotionality, testosterone and behavior, human sex differences, human aggression, grief and loss, and evolutionary psychology. He is also the author of several books, including The Behavioural Biology of Aggression (1988), The Nature of Grief (1999), and Sex and Gender (2nd edition, 2002). He is a former President of the International Society for Research on Aggression (ISRA), and is a Fellow of the British Psychological Society. 


\section{Aggression between same-sex individuals}

\subsection{Sexual selection as an explanatory framework for human aggression}

2.1.1. Principles of sexual selection. Sexual selection involves the choice of members of one sex by the other, and competition by members of one sex for access to the other (Darwin 1871/1901). The more competitive and aggressive sex is usually the male, particularly in mammals. Sexual selection forms a basis for understanding sex differences in aggression in animals, although it is not the only explanation (Ralls 1976; Selander 1972). Trivers (1972) proposed that the reason why males are typically the more competitive sex is their lower parental investment, defined as any contribution by a parent that increases an offspring's chances of survival and reproduction, while reducing the parents' ability to produce further offspring. Examples include the energy expended in gamete production (Bateman 1948), and feeding or guarding the young. The initial greater contribution by the female to the gametes will make desertion to seek access to other mates a more beneficial option for males than for females, providing that one parent can care for the offspring. ${ }^{2}$ The sex showing higher parental investment becomes a limiting resource, the other sex competing for reproductive access. Competition can take forms other than direct aggression, for example, sperm competition, or features that aid mate attraction, or securing a dispersed resource (Andersson 1994, pp. 10-13; Archer 1988, pp. 106-107).

When parental care by both sexes is required, inter-male competition will be countered by the male's greater paternal investment, as occurs in monogamous birds. Where the female can leave the male to brood the eggs and care for the young alone, as occurs in polyandrous wading birds, females will be the more competitive, and the larger and more aggressive, sex (Jenni 1974). This reversal of the usual sex differences provides crucial support for Trivers' theory. In mammals, fertilization is internal and the female feeds the developing offspring, so that parental investment is further female-biased, and polygyny is the usual outcome. In terrestrial mammals, this is typically associated with inter-male aggression, accompanied by large size and musculature, and a greater variation in male than female reproductive success (Andersson 1994).

Differential reproductive rate can be regarded as a more fundamental principle driving sexual selection than parental investment (Clutton-Brock \& Vincent 1991). It reflects the rates at which males and females are able to mate again, after producing offspring (and therefore is closely related to parental investment). Differences in reproductive rate were associated with sex differences in mating competition in 29 species where the male shows some parental care (Clutton-Brock \& Vincent 1991), and females had higher reproductive rates than males when the usual sex difference was reversed (i.e. when females were larger and more competitive than males).

The basic principles of sexual selection are complicated by ecological constraints on the degree to which one sex can compete for, and monopolize, access to the other sex (Emlen \& Oring 1977). Where resources that are important for reproduction are located in one place, there will be accentuated competition for access to the sex with the lowest reproductive rate, usually the female, and polygyny is more likely. Where resources are widely distributed, the potential for reproductive competition is less, and monogamy is more likely. These principles also apply to variation within a species. For example, the mating system of the dunnock (Prunella vulgaris) varies from polygyny to monogamy to polyandry (Davies \& Hartley 1996; Davies \& Lundberg 1984), depending on the food distribution, which affects female range size. Underlying these associations is the ability of males to control access to females, or more precisely, the concept of operational sex ratio (OSR; Emlen \& Oring 1977 ) - the ratio of sexually active males to fertilizable females at any given time and place. This will be biased in the female direction as a consequence of males being excluded from the breeding group by higher mortality, or being ejected as a result of competition, or maturing more slowly (Clutton-Brock \& Harvey 1977), and in the male direction if females only gradually become fertile or available to males (e.g., Smuts 1987b). The OSR is essentially an index of the degree of male competition for mates, and is associated with greater variance in male than female reproductive success.

Pomiankowski and Møller (1995) found greater variability in sexually selected than non-sexually selected traits in males than females from a range of species, and they attributed this to sexual selection producing traits exaggerated beyond those that were optimal for survival. An alternative explanation in species where there is some degree of paternal care is that males of many monogamous species retain traits associated with polygynous mating, and can therefore be regarded as facultatively polygynous (Andersson 1994, pp. 157-58; Trivers 1972). The degree to which this occurs depends both on the context (e.g., the availability of alternative mates) and the individual (e.g., the ability to attract alternative mates). Such individual differences are said to arise from the extent to which reproductive effort is concentrated on parental or mating effort, which is termed a conditional reproductive strategy (Gross 1996). It would lead to greater variation in sexually selected traits among males than among females.

\subsubsection{Sexual selection applied to human aggression.}

Because gestation in female mammals is internal, males must show a higher potential reproductive rate than females, and this is associated with being the competitive sex. The necessity of biparental care in some species will counter this and reduce the degree of sexual dimorphism. The extent to which this applies to humans is a matter of contention (Geary 2000), and humans have been regarded as basically monogamous or polygynous (sect. 2.1). However, even males of monogamous species are likely to be facultatively polygynous (Trivers 1972), and therefore reproductive competition is likely to be higher in men than women, irrespective of the basic human mating pattern.

Daly and Wilson $(1988 ; 1990)$ applied the principles of sexual selection to human homicide, which they regarded as indicative of the strength of aggressive dispositions in different individuals and under different circumstances. They viewed the much higher frequency of male than female same-sex homicides, and the concentration of these among men with few resources (sect. 1.8), as consequences of sexual selection. An alternative evolutionary view (Campbell 1999) explained the lower incidence of women's engagement in risky and violent aggression in terms of their relatively greater parental investment, 
which increases the importance of remaining alive to rear their offspring. As a consequence, women have evolved a psychological disposition to be more risk-averse. This can be viewed as either an alternative to the sexual selection view, which concentrates on male competition, or as complementary. It is still derived from unequal parental investment, and it generates very similar predictions to sexual selection.

The basic principle underlying all evolutionary explanations of aggression, including sexual selection, is a cost-benefit analysis, in which the costs and benefits of behavior are determined by natural selection. For male aggression, the benefits will be successful reproduction, and the costs those resulting from injury. For men with few resources, successful reproduction may only be possible if they challenge other men and risk injury in escalated encounters (Daly \& Wilson 1988; 1990): consistent with this, an evolutionary simulation demonstrated the increasing payoffs for risky (dangerous) tactics as the value of victory increased (Daly \& Wilson 1988, pp. 164-65). For women, access to a mate is less dependent on within-sex competition, and they typically have more to lose in terms of reproductive fitness from potentially damaging confrontations (Campbell 1999). The emphasis in these explanations is on higher potential reproductive benefits for males, and higher potential costs for females, of damaging aggressive encounters. It follows that the sex difference would be largest for dangerous forms of physical aggression, and be greater for physical than for direct verbal aggression. Indirect aggression is less dangerous in terms of inviting immediate retaliation, and has therefore been viewed as a lower-cost form of aggression, typically used more by females (Björkqvist 1994; Geary 1998; Hess \& Hagen 2006). The cost-benefit analysis locates the source of the sex difference in the damaging nature of physically aggressive encounters and would not predict a difference in features of aggressive motivation, such as the ease with which the two sexes are aroused to anger.

Typically, evolved dispositions such as those underlying a sex difference in aggression should not have to rely on socialization practices that could vary from culture to culture. How and when they first occur in development is an issue that is not precisely specified by a functional explanation, which primarily addresses adaptive significance. As Darwin (1871/1901) noted, sex differences may be small or minimal before reproductive maturity. The sex difference in size and strength conforms to this pattern, developing at puberty under the control of testosterone (Tanner 1989). Some researchers have suggested that testosterone controls the greater male physical aggression in humans (e.g., Book et al. 2001), following the link found in many mammals and birds (Archer 1988, pp. 135-42). An alternative is that the sex difference in direct aggression begins early in life (Bjorklund \& Pellegrini 2000), before the cumulative impact of gendered social influences, possibly as a result of a direct or indirect effect of prenatal androgens (Berenbaum \& Resnick 1997; Pasterski et al. 2007). It would be associated with other early-developing dispositional sex differences, such as those in activity (Archer \& Lloyd 2002, p. 74; Campbell \& Eaton 1999; Eaton \& Enns 1986), and social preferences for larger competitive groups rather than smaller, more supportive ones (Geary et al. 2003). Since sex-segregation, and the relationship styles accompanying this, occur early in life (Archer 1992a; Maccoby 1988; 1998; Pellegrini 2004), we would expect sex differences in aggression to occur then too. A further possibility, not necessarily inconsistent with an early emergence of sex differences, arises from the finding that males of many polygynous species delay risky encounters with older males until they are large enough to compete effectively with them (Andersson 1994). If this applied to humans, the peak years for high-risk confrontations would be when young adults become physically mature and enter the mating arena (Geary 2002). Overall, the most likely prediction from a sexual selection perspective is an early emergence of sex differences in aggression combined with a peak in risky competition during young adulthood, with a possible influence of pubertal testosterone.

Just as the course of development cannot be precisely specified from an evolutionary explanation, nor can the precise mechanisms underlying human sex differences in aggression. Sexual selection does, however, imply the following: (1) that the sex differences are not wholly the result of a general-purpose learning mechanism, although this is likely to be involved; (2) that there are sexually dimorphic neuroendocrine mechanisms underlying aggression, accompanying other aspects of sexual dimorphism, such as size and strength; (3) that the mechanism is unlikely to reside in a general sex difference in responses to frustration or in ease of arousal to anger; (4) that the sex difference is more likely to involve either greater risktaking by males or more fear of physical danger by females: either or both of these would represent the way that the motivational system underlying aggression had responded to evolutionary costs and benefits. These would represent basic predispositions that could be modified in development (sect. 2.5), or overridden by environmental contingencies (sect. 2.8).

Based on Trivers' analysis (sect. 2.1.1), sexual selection theory can also predict individual differences among men according to their relative specialization for mating or parental effort. One prediction is that there should be a coherent set of individual differences associated with mating or parental effort; a second is that variability in sexually selected traits such as physical aggression should be greater in men than in women, whereas traits that are not sexually selected, such as anger, should not show sex differences in variability.

Sexual selection theory also predicts variability in sex differences in aggression as a consequence of social conditions affecting the cost-benefit contingencies of reproductive competition. For example, inter-male competition will be accentuated where resources are scarce, and where there are fewer females than males of reproductive age; that is, where the OSR is high. Where the OSR is in the other direction, with fewer males, we would expect greater female competition and overt aggression.

Table 1 provides a summary of the main points of the predictions set out in this section, together with the sections that consider the evidence relating to them.

\subsection{Social role theory as an explanatory framework for human aggression}

2.2.1. Principles of social role theory. The main alternative explanation of the origins of human sex differences in social behavior is the "biosocial"3 reformulation (Wood \& 
Archer: Sex differences in aggression

Table 1. Summary of predictions about sex differences in aggression from sexual selection and social role theories

\begin{tabular}{|c|c|c|c|}
\hline & Relevant section & Sexual selection theory & Social role/biosocial theory \\
\hline $\begin{array}{l}\text { Magnitude and nature of the } \\
\text { sex difference }\end{array}$ & 2.4 & $\begin{array}{l}\text { In accordance with the degree of } \\
\text { physical danger, the largest } \\
\text { differences will be in physical } \\
\text { aggression, followed by direct } \\
\text { verbal, with indirect aggression } \\
\text { showing no difference or more by } \\
\text { females; no difference for anger. }\end{array}$ & $\begin{array}{l}\text { Magnitude will be modest; larger } \\
\text { difference for physical than for } \\
\text { psychological aggression (verbal and } \\
\text { indirect). Presumably, no difference } \\
\text { for anger. }\end{array}$ \\
\hline Development & 2.5 & $\begin{array}{l}\text { Early emergence of sex difference in } \\
\text { direct aggression; peak in damaging } \\
\text { and risky competition during young } \\
\text { adulthood. }\end{array}$ & $\begin{array}{l}\text { Sex difference should start small and } \\
\text { increase with age through } \\
\text { childhood, coincident with the } \\
\text { cumulative influence of } \\
\text { socialization. }\end{array}$ \\
\hline Mediators & 2.6 & $\begin{array}{l}\text { These will reflect functional } \\
\text { principles, for example, greater } \\
\text { risk-taking by males or greater fear } \\
\text { of physical danger by females, or } \\
\text { both. }\end{array}$ & $\begin{array}{l}\text { Internal mediators should follow from } \\
\text { the characteristics of gender roles } \\
\text { (e.g., empathy, fear of retaliation, } \\
\text { guilt, anxiety associated with } \\
\text { aggression). They arise from general } \\
\text { learning mechanisms associated } \\
\text { with gender roles. } \\
\text { Biosocial model indicates that roles } \\
\text { can vary in response to role-related } \\
\text { costs and benefits. }\end{array}$ \\
\hline Individual differences & 2.7 & $\begin{array}{l}\text { There will be a coherent set of } \\
\text { individual differences (including in } \\
\text { aggression) among males, } \\
\text { reflecting greater or lesser } \\
\text { emphasis on mating than parental } \\
\text { effort. A consequence of these } \\
\text { differences will be greater male } \\
\text { than female variability in physical } \\
\text { aggression. }\end{array}$ & $\begin{array}{l}\text { Consistent individual differences } \\
\text { would arise from differences in } \\
\text { internalization of gender roles, and } \\
\text { from the influence of specific roles; } \\
\text { no consideration of possible sex } \\
\text { differences in variability. }\end{array}$ \\
\hline $\begin{array}{l}\text { Variability in response to } \\
\text { environmental conditions }\end{array}$ & 2.8 & $\begin{array}{l}\text { Variability across local conditions, } \\
\text { cultures and nations is expected to } \\
\text { reflect resources that are important } \\
\text { for reproduction, principally access } \\
\text { to the mates and the status and } \\
\text { resources important in this process. }\end{array}$ & $\begin{array}{l}\text { There should be variations in women's } \\
\text { level of aggressiveness } \\
\text { accompanying: (1) changes in role } \\
\text { salience; (2) cross-cultural variations } \\
\text { in women's relative emancipation; } \\
\text { and (3) changes over historical time } \\
\text { in women's roles and status. }\end{array}$ \\
\hline
\end{tabular}

Eagly 2002) of social role theory (Eagly 1987; Eagly et al. 2000). The basic tenet of social role theory is that sex differences in behavior arise "from the societal division of labor between the sexes" (Eagly 1997, p. 1381). Although it is primarily the societal positions of men and women, as breadwinner and homemaker, and in the workforce of modern societies, that are important, social role theory acknowledges that the roles of men and women are complex. Thus, more specific roles, such as those in the family (e.g., father, grandmother) and in occupations (e.g., police officer, nurse) also contribute to sex differences in social behavior and to within-sex variations.

The biosocial reformulation of social role theory (Wood \& Eagly 2002) represents an extension of the earlier accounts to address issues raised in exchanges with evolutionary theorists (e.g., Archer 1996; Buss 1996; Eagly \& Wood 1999), specifically the origins of sex differences, and the principles through which men and women are distributed in societal roles. It is also wider in scope, incorporating evidence from social and physical anthropology.
The biosocial account explains how recurrent forms of the division of labor based on sex arose from an interaction between the requirements of the social and physical environment and constraints imposed by the mammalian method of reproduction and sex differences in size and strength. Thus, women's childbearing and nursing of infants, and men's greater size and strength, make it easier for women to perform certain activities and men to perform others. This explains the cross-cultural consistency in gender roles. The interaction between these biological differences and particular ecological, social, economic, and technological forces, explains the variability in gender roles. The behavior associated with the specific sex-typed social roles that emerge from these interactions form the basis of behavioral sex differences, including those in aggression.

Social roles are therefore viewed as being rooted in human history, but they originate from phylogenetic history in the form of reproductive and physical sex differences. Nevertheless, Wood and Eagly (2002) stated that 
their biosocial model does not assume a major role for sexual selection in producing psychological sex differences such as those in aggression. Their argument rested on two points: (1) that sexual selection operates "primarily" in polygynous mating systems; and (2) that other indications of sexual dimorphism in humans, such as size and canine teeth, are low compared with other primates. I return to these issues in section 3 .

The notion that certain activities are more efficient under particular circumstances implies cost-benefit maximization. Although this is superficially similar to the reasoning in evolutionary explanations (sect. 2.1.2), no explicit connection is made with reproductive success in the biosocial account (Wood \& Eagly 2002, p. 704). Instead, the terms "cost" and "benefit" refer to individual utility maximization (Wood \& Eagly 2002, p. 719), which is more in line with their meaning in the social sciences, to indicate that individuals are behaving according to their own estimation of the outcomes of their actions (e.g., Kirkpatrick \& Epstein 1992; Tedeschi \& Felson 1994).

The developmental processes identified in social role explanations are largely those described in social learning accounts of how socialization produces sex-typed behavior. This was the initial focus of psychological explanations of sex differences in behavior, and gender roles were an important feature of these (e.g., Maccoby \& Jacklin 1974; Sears et al. 1965). Social role theory incorporated social learning (e.g., Bandura 1973; Bussey \& Bandura 1999; Perry \& Bussey 1979) as one of one of several processes underlying the social transmission of gender roles (Eagly 1987, p. 31; Eagly et al. 2004, p. 270; Wood \& Eagly 2002, p. 717). According to social learning theory, gender-typed behavior occurs as a result of the incremental effects of influences by parents, teachers, peers, and the media on the children's developing self-concepts, beliefs, and motives (Leaper \& Friedman 2007).

A variety of processes studied by social psychologists have been identified as underlying the way that shared characteristics of roles influence men's and women's behavior (Eagly et al. 2004). These can be regarded as the proximate causes of sex differences in social behavior. They include expectancies, situational contingencies, incorporation into self-concepts, and the acquisition of different skills and beliefs. One straightforward way that gender roles may influence behavior is through the internalization of gender-stereotypic traits (Eagly 1987), whose content is derived from roles, in the form of feminine communal traits (e.g., helpful, kind, nurturant), and masculine agentic traits (e.g., dominant, self-reliant, aggressive). The traits are not only internalized but also operate externally as expectations of behavior by men and women.

Later versions of social role theory (Eagly et al. 2004, p. 280; Wood \& Eagly 2002, pp. 701-702) also included neuroendocrine mechanisms. It is known that a range of hormones, principally those concerned with reproduction, and with stress reactions, are responsive to inputs from the social environment. For example, testosterone levels respond to sexual stimuli, the outcomes of competition, and fatherhood (Archer 2006b). Wood and Eagly (2002) interpreted these as reactions to the demands of social roles.

In social role accounts, within-sex variations in sextyped behavior can arise in several ways. One of these is through differences in the extent to which individuals internalize the gender roles of their society (Eagly et al. 2004 , p. 280). Another is through the operation of specific roles, for example, family and occupational roles. Social role accounts also predict when external circumstances would accentuate or diminish sex differences. When gender roles are made more salient, the magnitude of the difference should increase. We would also expect sex differences to be influenced by variations in gender roles across cultures or historical times. Eagly and Wood (1999) found that sex differences in mate preferences were larger in nations where gender roles were more traditional. Attitudes among college students changed from 1970 to 1995, in the direction of more frequent endorsement of equal rights for women (Twenge 1997a), and in accordance with this, women's own endorsement of agentic traits also increased over these years (Twenge 1997b).

\subsubsection{Social role theory applied to human aggression.}

According to the original social role theory (Eagly 1987, pp. 71-73), sex differences in aggression occur because aggressiveness is a component of masculine roles, and because traditional feminine roles discourage it, although both sets of roles involve norms that encourage and discourage aggression under different circumstances. Specific masculine roles, such as the military, and training in skills for physical aggression, further accentuate the sex difference. The higher societal status of men provides another route to greater direct aggression, since people perceive higher status to be associated with agentic characteristics (Eagly \& Steffen 1984). Social role analyses view status as one attribute of gender roles, although others distinguish between roles and status as influences on sex differences (e.g., Conway et al. 2005).

The original social role theory (Eagly 1987) predicted that men should be more aggressive than women, to a moderate extent, consistent with other sex differences in social behavior. Although the main research on indirect aggression had not yet been published, Eagly (1987) distinguished physical from psychological aggression in relation to experimental studies. The first was defined as producing physical harm and the second psychological or social harm, including what was later termed indirect or relational aggression (Archer \& Coyne 2005). Like sexual selection, social role theory predicts larger sex differences for physical than psychological aggression, on the basis that physical aggression is more clearly linked with masculine roles. Social role theory makes no specific predictions about anger, although the emphasis on sex differences in aggressive responses to anger-arousing situations implies that it predicts no difference in anger.

There is usually no explicit treatment of when sex differences in aggression are first expected to appear in socialization accounts, and no indication of their subsequent progression, since the emphasis is on the mechanisms underlying gender-differentiated behavior. However, in view of the cumulative nature of the processes of learning, it is reasonable to expect, as others have (e.g., Baillargeon et al. 2007; Tremblay et al. 1999), that aggression should increase with age through childhood, and that this will be more marked for boys than for girls, leading to larger sex differences in aggression with age throughout childhood. From this, we would expect a gradual onset of sex differences, and aggression in general, during early 
childhood, consistent with a gradual and cumulative learning process (Keenan \& Shaw 1997; Tieger 1980).

From social role theory, we would expect internal mediators of sex differences in aggression to follow general and specific features of gender roles. General features would be the internalization of masculine or agentic traits, which includes those associated with aggression. Variables such as empathy, fear of retaliation, and guilt and anxiety associated with the consequences of aggression, have been viewed as mediators of women's lower levels of aggression, as consequences of internalized gender roles (Bettencourt \& Miller 1996; Eagly \& Steffen 1986). In the biosocial account, neuroendocrine mechanisms are viewed as playing a different role than in sexual selection explanations. They are the consequences of role-related activity, which may then help to orient men and women to role-related activities.

In social role accounts, within-sex variability in aggression would arise from differences in the internalization of gender roles, and in the adoption of specific roles. Variability as a consequence of different environmental conditions would largely follow variations in the extent to which gender roles are operating. Decreasing the salience of gender roles should lead to a much reduced sex difference in aggression (Eagly \& Steffen 1986; Lightdale \& Prentice 1994). We would expect larger sex differences in nations with more traditional gender roles, and for the difference to have declined over the last 50 years in Western nations, in accordance with the increase in gender role equality.

Table 1 also provides a summary of the main predictions set out in this section, together with the sections that consider the evidence relating to them.

\subsection{Sources of evidence}

Much of the evidence discussed in the following sections is taken from meta-analyses of sex differences in aggression, which provide systematic summaries of several hundred research reports, involving observations, and reports from self, peers, and teachers, of "real-world" aggression, and experimental studies, both laboratory and naturalistic. Self-reports and experimental studies mainly involve adults, whereas the other methods involve children. The findings are reported for the different types of aggression, and for different measurement methods (Table 2). The values are Cohen's $d$, which is the difference between male and female mean values, standardized in units of the overall standard deviation. If positive, it represents a higher value for males, and if negative it represents a higher value for females. The types of aggression sampled in these analyses were physical, verbal, and indirect, and one analysis of self-reported trait anger (Table 2). The methods used in laboratory and field experiments are not directly comparable with those used in the "realworld" studies, and the effect sizes for the sex differences tend to be smaller (Archer 2004), but they provide a useful alternative to self-reports for adult ages where observations and peer reports are no longer practical options.

Self-reports (194 samples: Archer 2004), typically involve young adults. Laboratory and field experimental studies (50 samples: Eagly \& Steffen 1986; 107 samples: Bettencourt \& Miller 1996) are likely to include a high proportion of college students. In Archer's (2004) meta-analyses, there
Table 2. Sex differences in types of aggression for different methods of assessment

\begin{tabular}{|c|c|c|c|c|}
\hline Method and study & Physical & Verbal & Indirect & Anger \\
\hline $\begin{array}{l}\text { Experimental } \\
\text { (Eagly \& Steffen } \\
\text { 1986) }\end{array}$ & .40 & .18 & & \\
\hline $\begin{array}{l}\text { Experimental } \\
\text { (Bettencourt \& } \\
\text { Miller 1996) }\end{array}$ & .48 & .36 & & \\
\hline $\begin{array}{l}\text { Self-reports } \\
\quad \text { (Archer 2004) }\end{array}$ & .59 & .19 & $.05^{\mathrm{a}}$ & .035 \\
\hline $\begin{array}{l}\text { Observations } \\
\quad(\text { Archer 2004) }\end{array}$ & .55 & .09 & & -.45 \\
\hline $\begin{array}{l}\text { Peer-reports } \\
\quad(\text { Archer 2004) }\end{array}$ & .80 & .55 & & -.10 \\
\hline $\begin{array}{l}\text { Teacher-reports } \\
\text { (Archer 2004) }\end{array}$ & .33 & .24 & & -.21 \\
\hline $\begin{array}{l}\text { Combined }^{\mathrm{b}} \\
\quad \text { (Knight et al. 1996) }\end{array}$ & .91 & .46 & & \\
\hline $\begin{array}{l}\text { Combined }^{\mathrm{b}} \\
\quad \text { (Knight et al. 2002) }\end{array}$ & .59 & .28 & & -.07 \\
\hline
\end{tabular}

Note: Values are weighted mean effect sizes $(d) . d$ values from Archer's studies are those with outliers removed.

${ }^{a}$ This value excludes values from the indirect hostility scale of the Buss-Durkee Hostility Inventory, which measures a composite of indirect and displaced aggression (see Archer 2004).

$\mathrm{b}$ This category refers to a combination of measurement methods (all of those listed above, and projective methods in the case of the 1996 source). The 1996 source was a reanalysis and extension of a previous analysis (Hyde 1984; 1986).

are 64 samples using observations, with an age range of 1.8 to 13.2 years; 51 samples using peer reports, with an age range of 5 to 17 years; and 40 samples using teacher reports, with ages ranging from 3 to 15 years. Most studies are from the United States, although self-report studies come from 23 other countries.

These summaries of sex differences in aggression refer to measures for which the sex of opponent is unspecified. Observational studies of children ${ }^{4}$ that do not specify the sex of the opponent are likely to involve same-sex encounters, since children tend to interact in sex-segregated groups (Archer 1992a; Maccoby 1988; 1998; Pellegrini 2004). Self-report methods for studying adults' aggression do not typically specify the sex of the opponent. Where they do (Gergen 1990; Harris 1992; Hilton et al. 2000; Richardson \& Green 1999), the values for same-sex physical aggression are very similar to those found in general questionnaires, and the values for opposite-sex encounters are in the female direction, consistent with findings for physical aggression to an opposite-sex partner (Archer 2000a). It is therefore reasonable to conclude that general questionnaires are largely measuring same-sex aggression (Archer 2000; 2000b; 2004) ${ }^{5}$

Meta-analyses have the advantage of precision, but they are limited to the data-bases used, which are typically from studies of aggression between individuals in Western societies. Evidence from other sources is therefore included, for example, criminological sources in relation to changes with age (sect. 2.5), and psychological studies for possible causal mechanisms (sect. 2.6). 


\subsection{Overall pattern of sex differences in aggression}

The overall pattern of sex differences in aggression enables an assessment to be made of the prediction, from sexual selection, that the magnitude of the sex difference will follow the cost or danger involved. Thus, higher $d$ values in the male direction are expected for physical aggression, a lower one in the male direction for verbal aggression, no sex difference or one in the female direction for indirect aggression, and no sex difference in anger. Table 2 shows the values for the three forms of aggression, for various measurement methods, and for trait anger. Across a range of measures at different ages, $d$ values were higher for physical than for verbal aggression, which were still consistently in the male direction, whereas those for indirect aggression were in the female direction in childhood and adolescence, and very slightly in the male direction for adults. Values for the selfreported experience of anger were around zero, indicating no sex difference, which is consistent with the results for anger in a meta-analysis of temperament in childhood (Else-Quest et al. 2006).

These findings support the view that the magnitude of sex differences follows the degree of immediate risk the actions entail (Archer 1994; Björkqvist 1994). Statistics on the proportion of men and women using weapons, and killing members of their own sex, are also consistent with this position. In several surveys of United States youth, reported in Archer (2004), 80\% of those who reported carrying a weapon were male. Aggregating data from 20 studies of nearly 14,000 same-sex homicides (Daly \& Wilson 1990) showed an even higher sex difference, with $97 \%$ of the perpetrators being male.

Of the meta-analyses listed in Table 2, only Archer (2004) sampled studies outside North America, and most studies in this analysis did come from the United States. There were, however, sufficient studies from other nations to show that the effect size was consistently in the male direction for physical aggression across 13 nations for self-reports, 9 for observations, and 5 for peer reports. Practically all the nations involved were industrial states with an effective rule of law. Historical evidence indicates that, in pre-industrial societies, men have the capacity for intense inter-male competition, which is likely to take a physical form, whereas in modern industrial states they typically use other ways of competing for resources ${ }^{6}$ (e.g., Courtwright 1996; Daly \& Wilson 1988; 2001; Eisner 2004).

Another limitation of the meta-analytic evidence is the emphasis on aggression between individuals. There is little systematic evidence on relative male and female involvement in coalitional aggression, which also occurs in chimpanzees (e.g., Wilson \& Wrangham 2003). It has been greatly extended in humans, in the form of raiding parties, primitive warfare in pre-industrial societies (Keeley 1996), gangs, organized crime, and the military in modern nations. These are all activities typically involving men. Keeley (1996) concluded, from several extensive cross-cultural surveys, that in non-state societies, intergroup warfare typically occurred more or less continuously, involving the mobilization of up to $40 \%$ of the male population, and the death of around $30 \%$ of the young men. It is possible that, as a consequence of their participation in group aggression, men's behavior and cognition are more intergroup-oriented than women's (van Vugt et al. 2007). Men (but not women) increased altruistic group contributions during intergroup competition (van Vugt et al. 2007), and they showed more risky decision choices when in the presence of other men (Daly \& Wilson 2001).

\subsection{Age trends in sex difference in physical aggression}

In section 2.1.2, I indicated some broad predictions from sexual selection theory concerning the development of sex differences in aggression. They would not be solely determined by cultural learning throughout childhood; they would be subject to some biological developmental influence, either early in postnatal life, or at puberty; and they would be largest in young adulthood. From a socialization perspective, we would expect sex differences to be small or nonexistent at preschool ages (see Keenan \& Shaw 1997), and to increase throughout childhood, reflecting the cumulative influences of gendered learning.

Three lines of evidence show that the sex difference begins early in life, and can be substantial at young ages, supporting an evolutionary analysis (Bjorklund \& Pellegrini 2000). First, individual observational studies (Table 3) show that sex differences in physical aggression are large early in life. Second, although Keenan and Shaw (1997) claimed that there was no sex difference in direct aggression for toddlers, on the basis of a narrative review of five observational studies, a meta-analysis of the same studies showed a significant difference $(d=.44 ; p=.003$ : Archer \& Côté 2005). Third, a longitudinal study of mother's reports of children's physical aggression at 17 and 29 months found sex differences at the first of these times, with no change in magnitude at 29 months (Baillargeon et al. 2007). The large-sample longitudinal study of Tremblay et al. (1999), represented in Figure 1 here, also found that the sex difference in physical aggression was present at 2 years of age, and that physical aggression showed a peak between 2 and 3 years of age, followed by a decline in the childhood years. They concluded that socialization must largely entail learning to inhibit physical aggression, rather than learning it from role models.

A meta-analysis of age trends for self-reported physical aggression showed no increase from before to after puberty $(6-11$ years: $d=.56 ; 12-17$ years: $d=.46$ : Archer 2004), coincident with the action of testosterone on reproductive physiology and secondary sexual

Table 3. Sex differences in physical aggression in four observational studies at young ages

\begin{tabular}{lllc}
\hline \hline Study & Sample & Mean age (yr) & $d$ value \\
\hline Sears et al. (1965) & 40 & 4 & 1.27 \\
McGrew (1972) & 30 & 4 & 1.29 \\
Hay et al. (2000) & 66 & 2 & .66 \\
Campbell et al. (2002) & 56 & 2.3 & $1.42^{\mathrm{a}}$ \\
\hline \hline
\end{tabular}

Note: $d$ values were calculated using DSTAT (Johnson 1989), and have previously been used for a meta-analysis (Archer 2004) or reported in a book chapter (Archer \& Côté 2005).

a This value was for "grabbing another child's toy." 


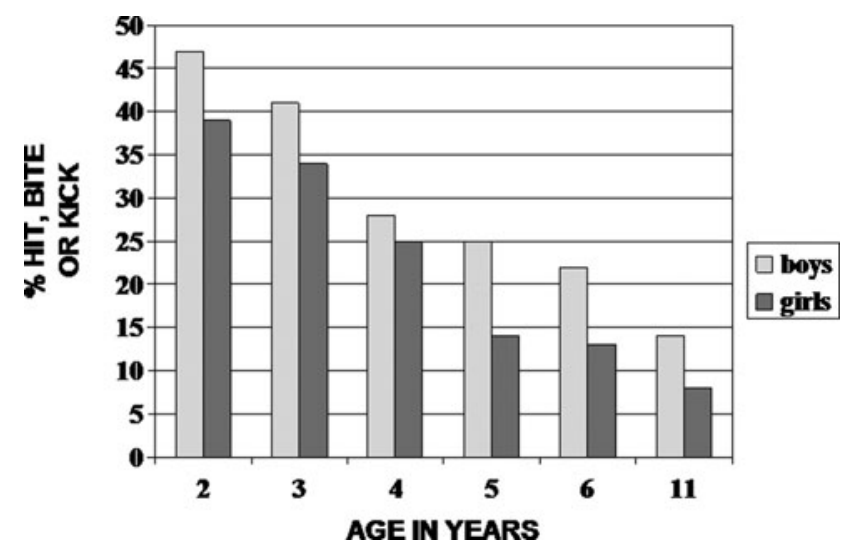

Figure 1. Changes in physical aggression of boys and girls with age (mothers' reports).

Source: Tremblay et al. (1999).

characteristics (Tanner 1989). Consistent with this, several longitudinal studies showed no increase in aggression for boys at puberty, and two others showed no link between testosterone levels and physical aggression at puberty (Archer 2006b).

Crime figures from a variety of historical times and cultures (Courtwright 1996; Daly \& Wilson 1990; 2001; Eisner 2003; Quetelet 1833/1984), show that men's involvement in violent crimes, and in same-sex homicides, is highest between the ages 18 and 30 years. This is as expected if they represent reproductive competition that is delayed until men have attained optimum size and strength (sect. 2.1.2). Effect sizes for sex differences in self-reported physical aggression are also largest in the 18-21 and 22-30 age categories (Archer 2004).

\subsection{Mediators of sex differences in physical aggression}

The implications of sexual selection for the mechanisms underlying sex differences in aggression are that they are likely to involve sexually dimorphic neuroendocrine mechanisms, and that they will be concerned with variables such as risk-taking, lack of inhibition, or fear of physical harm (sect. 2.1.2). The available evidence (sect. 2.5) did not support the view that male escalated aggression arises at puberty as a result of increased testosterone levels. Nevertheless, temporarily raising a woman's level of testosterone to that of a man enhances her cardiac defense reflex to angry (but not other) faces, in a masked Stroop test (van Honk et al. 2001). The rationale behind this procedure is that a greater initial reaction to an angry face is associated with threat rather than appeasement responding (Putman et al. 2004). A subsequent functional MRI (fMRI) study (Hermans et al. 2008) showed that in response to an angry face there was extensive activity in a range of cortical and subcortical brain areas known to be associated with aggression in nonhuman mammals. Further, administering testosterone affected the responses in these areas, primarily by increasing the excitability of subcortical structures involved in regulating aggression. Further studies have shown that laboratory measures of two suggested mediators of the sex difference in aggression, fear and empathy, are also reduced by administering testosterone to women (Hermans et al. 2006a; 2006b). These findings seem to indicate a direct influence of testosterone on brain mechanisms underlying aggression, at least in one specific aggression-related measure, and also an influence on two attributes that may mediate direct aggression. It is not easy to see how this research fits with the lack of a testosterone-induced increase in aggression at puberty, or the evidence that sex differences in aggression begin early in life. It may be that the brain areas are affected by pubertal testosterone, but that they only gradually manifest themselves in overt behavior over subsequent years.

Evolutionary analyses (sect. 2.1.2) emphasize greater risk-taking by males than females as underlying the differences in their aggression. Overall, men show more risktaking than women (Byrnes et al. 1999), although the sex differences are greater for certain types of risk: Effect sizes are highest for risks such as driving, drug and alcohol use, gambling, and everyday risks such as crossing a road (Byrnes et al. 1999; Daly \& Wilson 2001; Harris et al. 2006; Pawlowski et al. 2008; Zuckerman \& Kuhlman 2000). A mediator of these differences may be a sex difference in a related characteristic, such as fear (Byrnes et al. 1999; Campbell 1999), impulsivity (Campbell 2006), or sensation-seeking (Wilson \& Daly 2006; Zuckerman 1994; Zuckerman et al. 1993).

A study by Harris et al. (2006) suggests that several variables may underlie men's greater risk-taking: Men anticipated being less upset or harmed by possible negative outcomes; they judged negative consequences as being less likely to occur; and they reported higher enjoyment of risky activities. Applied to physical aggression, that would mean women anticipate greater harm from its consequences, which is highlighted in Campbell's (1999) evolutionary analysis and in social role theory (Eagly \& Steffen 1986). Women would also view such negative consequences as being more likely to occur, and show less enjoyment of physical aggression than men: This would fit the very large sex difference found for children's enjoyment of physical aggression (Benenson et al. 2008).

Campbell (1999; 2006) argued that higher levels of fear experienced by women in confrontational situations could mediate the sex difference in physical aggression. Consistent with this, the sex difference in fear occurs at an early age (Else-Quest et al. 2006), and is pronounced in situations involving physical danger. In experimental studies of adults (Bettencourt \& Miller 1996; Eagly \& Steffen 1986), the sex difference in aggression was higher under conditions of greater danger. Campbell (2006) also considered a related process, effortful control, as another possible mediator of sex differences in physical aggression. This is the ability to suppress a dominant (and immediately attractive) response in favor of a subdominant (and later beneficial) one. It shows a large sex difference in favor of girls from early in postnatal life (Else-Quest et al. 2006).

Social role theory predicts that sex differences in aggression will vary according to a number of external and internal variables associated with gender roles. Both guilt and anxiety about others' suffering, and exposure to danger to the self, are important aspects of internalized gender roles, corresponding to the fear and risk-taking identified in evolutionary analyses, and to empathy, which also features in evolutionary accounts. Eagly and Steffen (1986) found that women viewed their aggression as more likely to pose a danger to themselves than men 
did, and the extent of the perceived danger predicted the magnitude of the sex difference. Meta-analyses of experimental studies (Bettencourt \& Kernahan 1997; Bettencourt \& Miller 1996; Eagly \& Steffen 1986) found that women showed more empathy with the victim of their aggression (i.e., more anxiety and guilt after aggressing), than men did. For empathy to act as a mediator of the sex difference in physical aggression, it would have to operate to a greater extent as the level of escalation increased, as indeed it could as the consequences for the other person will be greater. It would have to be minimized in cases of indirect aggression, where the victim's emotional distress may be less apparent. It would also have to be lessened for aggression between partners where there is no sex difference in physical aggression in Western nations (sect. 4). This would be possible in cases where male suffering is minimized or disregarded (Felson 2002).

Another aspect of social role theory concerns the expectancies associated with status differences between men and women. These expectancies can either be internalized or reside in the others' expectations. Conway et al. (2005) found that expectancies for men's and women's aggression were similar to those for people differing in status. Lowstatus individuals were, like women, viewed as experiencing more guilt and anxiety, and causing less harm by aggressing, than were high-status individuals. Low-status individuals were also viewed as less physically aggressive. This study concerned the expectancies attributed to people by others. If they also represent internalized expectancies, as suggested by social role theory, they could guide the behavior of men and women, and therefore act as mediators of sex differences. One problem with this view is that cross-cultural and historical evidence has repeatedly shown that lowerstatus individuals with little to lose are those who are most inclined to engage in physical aggression (sect. 2.7).

Possible mediators of sex differences in aggression need to be viewed in terms of their social and developmental context, as contributors to the outcome of a developmental sequence arising from the interaction of internal and external influences. Individual components coalesce to produce the typical outcome only when embedded in a typical male or female sequence of development. For example, it is possible that prenatal testosterone alters predispositions for fear of physical danger, and for activity and play preferences, which manifest themselves in overt behavior only through interactions with other boys. The behavior so produced would then become viewed in terms of the gender roles operating in that culture, which will themselves influence behavioral development. Similarly, testosterone secretion at puberty may produce the dispositions shown in laboratory studies, but these produce sex differences in escalated physical aggression only under the social conditions that are manifest in early adulthood. Such specific dispositions will also be subject to activating and inhibiting social influences operating throughout development. For example, a culture that emphasizes the need to respond to the slightest provocation (the "culture of honor": sect. 2.8) is activating, and an effective rule of law is inhibiting. This perspective on mediators of sex differences in aggression involves social roles as both emerging from the interaction of dispositions and the social environment, and also feeding into this process.

\subsection{Within-sex individual differences in physical aggression}

A prediction derived from sexual selection is that, in species where there is parental investment, there will be individual differences among males reflecting a different emphasis on mating or parental effort. A number of studies show that individual differences in measures indicative of mating versus parental effort are associated with testosterone levels (Archer 2006b), and with a range of antisocial activities and behavioral and personality variables, including dominance and aggressiveness. These appear to be long-term dispositions, present before puberty.

Such individual differences among males will lead to greater variability among males than females in traits such as physical aggression, but not in those on which the sexes do not differ, such as anger and self-esteem. Archer and Mehdikhani (2003) tested this prediction in a sample of questionnaire studies, and found that male variability was indeed significantly greater than female variability for physical aggression but not for anger or self-esteem. Although these findings are consistent with the hypothesis that individual differences reflect variability in male mating strategies, a more direct test is needed to establish whether this is the case.

Social role theory makes no comparable predictions of differences in within-sex variability in aggression. It does identify a number of variables that will produce withinsex variability in both sexes. One is the different extent to which people internalize gender role norms (Eagly et al. 2004, p. 280). Consistent with this, self-reported physical aggression was highly correlated with agentic traits among a sample of men (Archer, submitted), and three other studies have found low to moderate associations between a measure highly related to self-reported aggression and endorsement of gender role traits (Campbell \& Muncer 1994; Campbell et al. 1993; Thanzami \& Archer 2005). Specific gender-related roles provide another influence on within-sex variability: Campbell and Muncer (1994) found that occupation (soldier or nurse) was a better predictor of a person's attributions about his or her own aggression than was the person's sex.

\subsection{Variability in sex differences in aggression in response to environmental conditions}

Sexual selection predicts variability in response to conditions that affect the extent of inter-male or interfemale competition, notably resources that are important for reproduction, such as access to mates, and the status and resources important in this process. Consistent with this, violence is higher among men who have few economic resources or prospects (Courtwright 1996; Daly \& Wilson 1988; Eisner 2003), and is lower among married men than those who are single, divorced, or widowed (Daly \& Wilson 2001). In an analysis of Japanese homicide figures, Hiraiwa-Hasegawa (2005) found that the rate of homicides by young males had declined considerably since 1955, and that this coincided with smaller families, an increase in GDP per person, and a high proportion of young adults in education, all indicators of greater resource availability. The annual rate of homicides for young males from 1960 to 1996 was inversely related to 
college enrollment and the degree of financial equality in the society. These influences are very different from those found to account for the more limited decline in violent crime in the United States in the 1990s (Levitt 2004), but are similar to the likely causes of the longer-term large decline in violent crime since the fifteenth century in Europe (Eisner 2003).

A simple prediction from the concept of Operational Sex Ratio (OSR; Emlen \& Oring 1977), the ratio of sexually active males to fertilizable females in the population (sect. 2.1.1), is that inter-male competition will be intensified where there are a greater number of males of reproductive age. Hudson and Den Boer $(2002 ; 2004)$ showed from historical records that where there had been high male-biased sex ratios (e.g., in nineteenth century China and medieval Portugal), there was social unrest, individual and collective violence, and expansionist military campaigns. The consequences of a low OSR were indicated by Campbell (1995), who reported localized high rates of physical aggression among young women where a large proportion of young men were not regarded as suitable mates as they had debilitating drug habits. Schuster $(1983 ; 1985)$ also documented escalated female aggression in Zambia and in China when there was competition for desirable men.

In these examples, environmental conditions accentuate the relative benefits of successful direct competition. In other cases, social forces operate in the opposite direction. One that has been particularly important in human history is the effectiveness of a rule of law. Where this makes men secure in their own safety and the security of their property, the costs of ignoring a challenge will be relatively low. There are also likely to be benefits from this course of action, such as avoiding injury and legal penalties. When there is no effective rule of law, and a man's possessions and livelihood can readily be taken by force, the costs of ignoring a challenge will be high. Consequently, establishing a reputation for effective retaliatory power is necessary, and not to do so is to risk one's personal safety, possessions, and family. A "culture of honor" (Nisbett \& Cohen 1996) emerges under these circumstances.

Social role theory predicts that there will be variations in sex differences in aggression accompanying differences in the salience or nature of men's and women's roles. Since provoked aggression is viewed as justified aggression, this justification was regarded as freeing women from the constraints normally imposed by gender roles (Bettencourt \& Miller 1996). Consistent with this, experimental studies showed a smaller sex difference in aggression when participants were provoked (Bettencourt \& Miller 1996). Another influence on the salience of roles is whether the behavior occurs in public or private settings, although the prediction that roles are more salient in public is complicated by the varied effects of different audiences: Experimental findings were ambiguous on this issue (Eagly \& Steffen 1986).

One particular study was regarded by its authors, and by Bettencourt and Miller (1996), as specifically supporting a social role explanation of sex differences in aggression. Lightdale and Prentice (1994) experimentally reduced role salience by creating a sense of anonymity and unaccountability regarding the opponent in a video-game task involving dropping bombs on an opponent following provocation. There was a striking reversal of the usual sex difference under these conditions $(d=-.41$, from the authors' Table 3) compared with those in which opponents were identifiable and known to one another $(d=.92)$. A more recent study (Evers et al. 2005) reported similar findings, but explained them in terms of the social appraisal of emotions rather than role salience. Men showed a higher aggressive response than women did when they expected to meet the person who had angered them $(d=2.42$, from the authors' Table 2$)$, but there was no sex difference $(d=.11)$ when people believed they would not meet the person who had angered them. An alternative explanation for both findings would be that the aggression required under the anonymous condition is indirect aggression, involving a lesser likelihood of anticipated retaliation (Björkqvist 1994). This would link the findings to a larger set of studies on indirect aggression (Archer \& Coyne 2005), and to Campbell's (1999) evolutionary view that emphasizes women's greater anticipation of negative consequences for their direct (but not indirect) aggression.

Social role theory predicts that sex differences will be larger when more traditional gender roles operate (e.g., Eagly \& Wood 1999). Although sex differences in physical aggression are available for a variety of countries (Archer 2004), and all show a difference in the male direction, they could not be used to test this prediction as the measures and samples were inconsistent. Lippa (in press) assessed the association between the relative empowerment of women in different nations and sex differences in the personality trait agreeableness, which is inversely related to aggressiveness (Gleason et al. 2004; Sharpe \& Desai 2001). He found lower sex differences where there were more traditional gender roles, the opposite of that expected if sex differences were accentuated by more pronounced gender roles.

Another prediction from social role theory is that there would be a lowering in the magnitude of sex differences in physical aggression coinciding with changes in gender roles in North America since the 1960s (Twenge 1997a; 1997b). Although there is no direct evidence on this, sex differences in the related characteristics of assertiveness and dominance did decrease during this time period (Twenge 2001). Women's assertiveness and dominance rose and fell over a longer time period, from 1931 to 1993, in accordance with changes in women's status and roles. From 1968 to 1993, there was a change in the sex difference in assertiveness from a moderately higher level in men $(d=.40)$ to a slight female advantage $(d=-.07)$. A direct investigation of changes over time in women's aggression in relation to changing roles is hampered by the lack of consistent measures and the availability of studies throughout the whole time period.

\subsection{Conclusions for sex differences in aggression between same-sex individuals}

The evidence reviewed in section 2.4 supports the first prediction from a sexual selection analysis that sex differences in aggression will increase in magnitude as the form of aggression becomes potentially more costly. Thus, physical aggression shows a substantial effect size in the male direction; verbal aggression a smaller one in the male direction; indirect aggression shows no sex difference, or is in the female direction; and there is no difference in anger. Weapons use and homicide statistics show 
Archer: Sex differences in aggression

even larger sex differences. The social role prediction that sex differences in aggression should be modest applies only to its less damaging forms, although social role theory does predict differences in effect sizes between physical and indirect aggression. Sexual selection provides a better fit with the overall pattern of greater sex differences for more risky and costly forms of aggression.

The finding that a sex difference in physical aggression occurs early in postnatal life, together with an increase in the magnitude of the sex difference in young adulthood, when there are also very large sex differences in violent crime and homicide, fits one of the two patterns expected from sexual selection. There is no evidence of an increase in aggression coinciding with puberty. The absence of a progressive increase in the size of the sex difference in physical aggression during childhood is inconsistent with an explanation in terms of the gradual differential learning of aggression by boys and girls, inferred from social learning accounts.

From sexual selection, we would expect mediators of the sex difference in aggression to follow functional principles, such as greater risk-taking by males and/or greater fear of physical danger by females. Although there are currently no direct tests of these as possible mediators, indirect evidence showed sex differences in these variables, consistent with this prediction, and some evidence that they were influenced by testosterone. Both greater risk-taking by men and fear of physical danger by women also feature as possible mediators in social role accounts, in this instance connected to masculine and feminine roles. I have outlined a developmental perspective that encompasses biological dispositions interacting with the social environment, with the resulting outcomes being reflected in social roles, which in turn influence the process of development.

There is evidence consistent with the prediction that there would be a coherent set of individual difference variables among males, reflecting greater specialization for mating versus parental effort. These variables are associated with testosterone levels. The greater variability in men's than women's physical aggression levels also supports this prediction. Social role theory does not predict greater variability for males than females. However, there is clear evidence that individual differences reflecting internalization of gender roles are associated with aggression-related measures, supporting predictions from social role theory.

Context-dependent variability is approached differently by sexual selection and social role theory. A number of studies suggest that those with few resources are the most prone to physical aggression, and that differences in the operational sex ratio accentuate competition in the more numerous sex: both of these suggestions are consistent with sexual selection analyses. Social role theory predicts that sex differences in aggression will be absent when roles are not salient. Two experimental studies were apparently consistent with this prediction, although the findings could be explained in terms of aggression being indirect when the aggressor had no prospect of meeting the victim. A cross-cultural analysis of sex differences in the personality trait agreeableness (which is inversely related to aggression) produced findings opposite to those expected from social role theory. However, it is clear that agentic characteristics in general, and assertiveness in particular, have changed in North America from the 1960s to the present, as gender attitudes became less traditional.

\section{Evidence for an adaptive complex produced by inter-male competition}

\subsection{Overview}

Section 2 indicated considerable evidence that was consistent with a sexual selection explanation of sex differences in aggression. In nonhuman mammals, greater male than female engagement in escalated aggression is typically accompanied by other sexually selected attributes, forming an adaptive complex associated with a polygynous mating system (Alexander et al. 1979; Clutton-Brock et al. 1977). This is characterized by higher male than female variance in reproductive success, which is equivalent to a measure of effective polygyny (Daly \& Wilson 1983). I consider the evidence for this among humans in section 3.2. The adaptive complex (sects. 3.3 to 3.6), involves several features: (1) specializations for inter-male encounters, such as threat displays, involving visual and vocal signals; (2) larger size, musculature, and strength, among males than females; (3) longer maturation rates in males than females; (4) greater male than female mortality rates, during both immaturity and adulthood, accompanied by a greater number of males than females conceived. Section 3.7 concerns variability in mating systems in relation to the adaptive complex.

\subsection{Polygyny and variance in reproductive output}

From a comparative primatological perspective (Fuentes 1999), humans do not fit the criteria of a monogamous species, and cross-cultural surveys show the existence of polygyny in most pre-industrial societies (Alexander et al. 1979; Ford \& Beach 1951; Murdock 1967). Alexander et al. (1979) argued that the widespread occurrence of monogamy in the world today is imposed on a more general pattern of polygyny, except in societies where ecological conditions are harsh. Betzig $(1986 ; 1992)$ used historical sources to show that whenever there was political and economic inequality, as was generally the case in the large historical empires, mating was effectively polygynous, even if marriage was legally only monogamous. Thus, throughout recorded history, powerful men typically had a very high number of sexual partners (Betzig 1986; 1992), which until recent times was reflected in their numbers of offspring (Daly \& Wilson 1988). This is maintained to some extent today. In modern Western nations where divorce is available, men who can afford a younger wife at the expense of an older one are likely to have the same effect on the variation in reproductive success as polygyny. In a study of a society where remarriage was common (Sweden), Forsberg and Tullberg (1995) found that remarriage produced increased numbers of offspring for men but not for women.

Several studies of pre-industrial societies enable figures to be calculated for male and female variance in reproductive success. Variance ratios were: 3.1 for the Brazilian Xante Indians (Salzano et al. 1967); 1.77 for the Dobe !Kung (from Howell 1979, p. 269); from 2.02 to 4.69 for Yanomamö (Chagnon 1979); and 2.76 for Aka pygmies (Hewlett 1988). All indicate effective polygyny. 


\subsection{Aggressive display features}

Primate males typically show ritualized threat displays, and these are probably associated with the higher costs of male than female fights (Smuts 1987a). Throughout primates, male facial hair growth is associated with threat displays (Andersson 1994, p. 345), increasing the apparent size of the lower part of the face (Guthrie 1970). Consistent with this are suggestions that the male beard is the consequence of selection for competition over rank and resources (Darwin 1871/1901; Guthrie 1970; Tanner 1989). The few empirical studies of this suggestion support it. Addison (1989) found that college students rated bearded men significantly higher than beardless men on aggressiveness, dominance, masculinity, and strength. Muscarella and Cunningham (1996) manipulated facial stimuli, and found that facial hair increased perceptions of aggression, although such faces were rated as less attractive and lower on social maturity.

Sell (2006) argued that the human angry face is an adaptation to accentuate facial cues indicating size and strength. Consistent with this, people can predict the lifting strength of young men from their faces (Sell et al. 2009). This applied to faces from their contemporaries, students from the University of California, Santa Barbara $(r=.39$ and $.45)$, and from two South American Amerindian groups $(r=.52$ and .47). Two features of the human face show sexual dimorphism, the brow ridges and the chin. Both are exaggerated in the angry face (Sell 2006): Ratings of "angry" and "masculine" were increased by lowering the brow ridges in androgynous prototypical faces with emotionally neutral expressions (Becker et al., 2007). It is likely that other sexually dimorphic features, such as neck size, contribute to male threat displays (Guthrie 1970), and that these constitute "honest advertisements" (Clutton-Brock \& Albon 1979) of fighting ability.

Weston et al. (2004, p. 416) referred to the paradox between "marked body size dimorphism, suggestive of strong sexual selection" in the human line and the absence of larger male canines, the other feature associated with inter-male competition in primates. Their analysis of 14 species of New World monkeys and apes showed that canine dimorphism is negatively correlated with another sexually dimorphic feature, facial width-toheight ratio. They argued that contrary to the absence of canine dimorphism being a sign of weak sexual selection, it is associated with strong selection for this alternative feature. Their subsequent analysis of a series of human skulls from infancy to adulthood confirmed that this is a sexually dimorphic feature in humans (Weston et al. 2007). Their preferred explanation was in terms of female choice, but a subsequent study of three samples of young men (Carré \& McCormick 2008) found that facial width-to-height ratio is associated with aggressiveness, suggesting that it may be a further cue for coercive power, or possibly for dispositional aggressiveness.

A range of male vertebrates use vocalizations as threat displays (e.g., Clutton-Brock \& Albon 1979; Davies \& Halliday 1978; McElligott et al. 1999; Mager et al. 2007), and these generally indicate fighting ability, or Resource Holding Power (RHP; Parker 1974a), which can be used to decide whether or not to fight. In human males, two changes occur to the voice at puberty, a lowering of the fundamental frequency, and a descent of the larynx, resulting in lowered formants and less formant dispersal. Evans et al. (2006) found that the first was associated with measures of shoulder-to-hip ratio, and the second with body size and shape. Artificially lowering the pitch of a man's voice increased ratings of his fighting ability by other men (Puts et al. 2006; 2007). Men who regarded themselves as physically dominant lowered their voice pitch in a competitive situation (Puts et al. 2006). These studies are consistent with the view that the deepness of the male voice is also an "honest advertisement" (Clutton-Brock \& Albon 1979) of fighting ability. In a hunter-gatherer group, men with deeper voices had higher reproductive success than those with higherpitched voices (Apicella et al. 2007).

\subsection{Size and strength}

Although females are larger than males in most animals, larger male size is typical of mammals (Andersson 1994; Darwin 1871/1901; Lindenfors et al. 2007). Human males are on average taller, weigh more, and have greater strength and musculature than human females. Alexander et al. (1979) summarized data from 93 societies from the ethnographic record, in terms of the ratio of male-to-female height. On average, men were $7.6 \%$ taller than women, with a variation of $4.7 \%$ to $11.6 \%$ between societies. A large-scale study using data from North Indian migrant workers from 1842 to 1916 found values of $7 \%$ to $8 \%$ for ages 15 to 40 years (Brennan et al. 1997). Data from nineteenth century British Columbia produced values ranging from 6 to $10 \%$ for seven adult age categories (Hall 1978). More recent evidence shows similar differences in the United States, Switzerland, and China. Effect sizes calculated from these studies are very large (Hall 1978: $d=2.67$; Kyle et al. 2005: $d=1.78$ to 1.85 ; Lindle et al. 1997: $d=1.14$; Luk et al. 2003, $d=5.95$; Pheasant 1983: $d=1.07$; Xiao et al. 2005: $d=2.24$ ).

Men are around $25 \%$ heavier than women of a similar age, and these are large differences when expressed as effect sizes (Kyle et al. 2005: $d=.87$ to 1.79 ; Lindle et al. 1997: $d=.60$; Luk et al. 2003, $d=.78$; Pheasant 1983: $d=1.15$; Xiao et al. 2005: $d=1.30$ ). Sex differences in fat-free body mass are even larger, with effect sizes reaching $d=2.36$ for a U. S. sample and $d=3.46$ for a Swiss sample (Kyle et al. 2005), with the male-to-female ratios reaching 1.40 in the Swiss sample and 1.43 in the U. S. sample,

Across 112 studies, Pheasant (1983) found that women's mean strength was $61 \%$ of that of men. Other studies have found similar or higher values (e.g., Battié et al. 1989; Luk et al. 2003; Xiao et al. 2005), with $d$ values from 1.45 to 3.09, depending on the sample and the strength measure (Battié et al. 1989; Xiao et al. 2005). Compared to women, men also have larger hearts and lungs, higher systolic blood pressure, lower resting heart rate, greater oxygen-carrying capacity, and greater ability to neutralize the products of muscular exertion (Tanner 1970).

These studies indicate considerable sex differences in muscle mass and strength, although they are smaller than in highly polygynous primates, where the male-to-female body mass ratio is above 1.5 (Plavcan 2000, p. 331; Plavcan \& Schaik 1997a). In humans, it is assumed that both paternal investment (Geary 2000) and male-male coalitions (Geary et al. 2003) have lessened the impact of inter-male competition, and with it the degree of dimorphism in strength 
and size, compared to more polygynous ancestral species. Estimates of the male-to-female body mass indices for Australopithecines range from the oldest, A. afarensis, at 1.52, to the more recent $A$. africanus, robustus, and boisei at 1.32, 1.26, and 1.40 (Plavcan 2000), which are similar to chimpanzees at 1.30 (Plavcan \& Schaik 1997a), but not much more than modern humans. If, as McHenry and Coffing's (2000) analysis indicates, the decreased dimorphism from Australopithecines to modern humans resulted from a relatively greater increase in female than male size, this suggests that it did not reflect a lessening of inter-male competition. However, all estimates of dimorphism in extinct hominids rely on fragmentary fossil remains (Plavcan \& Schaik 1997a), and should therefore be treated with caution.

\subsection{Maturation rates}

A further characteristic of sexual selection is bimaturism, males starting to reproduce at a later age than females. Andersson (1994) suggested that this occurs where size is important for inter-male competition: by delaying reproduction, males can avoid risky fights with older, larger, males. This reduction in male competition will have the effect of reducing the OSR (sect. 2.8), one consequence of which is to increase the degree of polygynous mating (Clutton-Brock \& Harvey 1977). Boys take around two years longer than girls to reach puberty (Tanner 1989), and a difference in maturation is apparent early in life: half-way through the fetal period, girls are three weeks ahead of boys (Tanner 1989).

\subsection{Mortality and conception rates}

Higher male than female mortality occurs in humans throughout life, both as result of greater male vulnerability to disease, stress, and injury, and higher risk-taking and violent behavior by men. Although the specific causes of death have changed throughout human history, and vary under different environmental conditions, the typical end result is greater male mortality (Kruger \& Nesse 2006), through a combination of greater engagement in risky activities and higher disease susceptibility. Analyzing mortality rates in U. S. statistics for 2000, at five-year intervals throughout the lifespan, Kruger and Nesse (2006) found that the peak male-to-female mortality ratio $(\mathrm{M}: \mathrm{F}$ MR) was in young adulthood (20-24 years), when the overall value was 3.01, and it declined thereafter. The curve for changes in the M:F MR with age was very similar to that for the age-crime curve. The ratio tended to be higher throughout life for external causes, whose peak value was 4.03, again during the 20-24 years age group. Kruger and Nesse also calculated M:F MR for a sample of chimpanzees and generally found higher values for males across the lifespan (1.43), again with a pronounced peak in young adulthood. Analysis of figures for Ache hunter-gatherers, where homicide was a major cause of death, showed an overall value of 1.77 , with the peak more evenly distributed throughout adult life.

Accompanying the greater male mortality throughout life is an unequal sex ratio at conception, leading to equal numbers of males and females in young adulthood. At conception, the ratio has been reported as between 110 and 160 human males for every 100 females, reduced to 105:100 at birth (Shettles 1961). Alexander et al. (1979) suggested that the sex ratio at conception reflects a long history of selection based on greater male than female mortality before maturity.

\subsection{Ecological influences on mating systems and sexual dimorphism}

The analysis by Emlen and Oring (1977) of the impact of environmental resources on mating systems (sect. 2.1.1) was applied by Alexander et al. (1979) to human mating systems. These authors did find that monogamous societies occurred in marginal or extreme habitats, as predicted, but that they also occurred in large and complex societies where monogamy was imposed as a form of social control. Alexander et al. (1979) created three categories, ecologically imposed monogamy, socially imposed monogamy, and polygyny, and applied these to 93 societies from the standard cross-cultural sample (Murdock 1967). They assessed the hypothesis that in the first of these, where nutrition is likely to be suboptimal, men will pursue a reproductive strategy involving greater parental than mating effort, which will be accompanied by reduced size and hence physical dimorphism. Their analysis of the ratio of maleto-female height was consistent with this, with mediumsized differences $(d=.58$ and .63 , from the authors' tables 15.4 to 15.6 ) between the first and the other two categories. However, a re-analysis of these data, and an analysis of a larger sample of 237 societies (Wolfe \& Gray 1982), showed that geographical region, rather than mating system, could account for the variation in height dimorphism across the societies. There were also problems with the measure of polygyny: When they used the original codes from Murdock (1967) for the larger sample of 216 societies, Gray and Wolfe (1980) found no association between polygyny and sexual dimorphism.

Low (1988) used multiple existing assessments of the degree of polygyny in a standardized cross-cultural sample of 93 societies, to test a proposed link between polygyny and pathogen stress (following Hamilton \& Zuk 1982). The reasoning was that when pathogen stress is severe, heritable pathogen-resistance will be a highly selected male trait. Pathogen-resistant males will therefore attract a disproportionate number of females, resulting in polygyny. Consistent with this, Low (1988) found a positive association between polygyny and pathogen exposure. A subsequent study (Low 1990) found the same association for all 186 societies in the ethnographic atlas (Murdock 1967). Whether these variations in polygyny are associated with variations in sexually selected features, such as height and aggression, remains to be assessed.

\subsection{Aggression and the sexually selected adaptive complex}

There are therefore a number of other features, besides aggression, that indicate the operation of sexual selection in humans. Greater variance in reproductive success for males than females indicates effective polygyny. There are vocal and facial features that indicate adaptations for threat displays in men, there is greater male size and strength, longer male maturation, higher male mortality, and a male-biased conception ratio. Taken together, these indicate a coevolved adaptive complex, associated with inter-male competition. 
The view that sex differences in size and strength are an evolutionary consequence of inter-male competition can be traced back to Darwin (1871/1901), and it has been a widely accepted view since then. Nevertheless, in their biosocial model (sect. 2.2.1), Wood and Eagly (2002) argued that a variety of selection pressures operating on females as well as males could explain these differences. Although animals do show a range of influences on size dimorphism (Andersson 1994; Ralls 1976; Selander 1972), a systematic review demonstrated the link between greater male size and success in fights and dominance contests in many animals (Andersson 1994). Plavcan and van Schaik (1997b) analyzed data from 86 extant anthropoid primates (New World monkeys and apes), finding that greater male-to-female size is associated with higher levels of inter-male competition $\left(r_{s}=.73\right)$. This shows a clear association between size dimorphism and inter-male competition across nonhuman primates. There is also evidence that size and strength are positively associated with men's history of physical aggression (Archer \& Thanzami 2007; Felson 1996; Sell 2005; Sell et al. 2005; Tremblay et al. 1998).

In terms of effect sizes, sex differences in features such as size and strength, and mortality, are considerably larger than those reported for physical aggression (sect. 2.4). For example, $90 \%$ of people can be classified successfully as male or female on the basis of their shoulder-to-hip width (Tanner 1989), or their lifting strength (Pheasant 1983), but the corresponding values from measures of physical aggression would typically be 62 to $73 \%$ (calculated from Rosenthal 1984, p. 131). These lower values may be because they are taken from people living in states with an effective rule of law, and who have alternative methods of competing, and because legitimized forms of aggression - for example, in the military, or in sports contests - are not included in these analyses (see Geary [1998] for further discussion of this point). If this is correct, such studies underestimate the magnitude of sex differences in aggression in other contexts.

\section{Physical aggression between opposite-sex partners}

\subsection{Explanatory frameworks}

So far, I have considered same-sex aggression, the focus of sexual selection and social role theory. In section 2.3, I indicated that the findings are different for aggression between members of the opposite sex. Most of the available data on physical aggression between opposite-sex adults involves married, cohabiting, or dating partners. Two main explanations dominate the literature. The patriarchal framework emphasizes the male nature of partner violence and views it as derived from the societal power of men, which is rooted in history (e.g., Dobash \& Dobash 1977-78; 1980; Walker 1989). This is a more limited explanation than social role theory, which seeks to explain the behavior of both men and women. In contrast, family relations researchers have been informed by large-scale surveys indicating that both sexes can be perpetrators and victims. Their accounts (e.g., Straus 1999; Straus \& Gelles 1988) therefore emphasize influences on both sexes, involving the frustrations of everyday living, a view that coincides with social psychological analyses
(Berkowitz 1993). Although the evidence strongly supports the family relations perspective, the patriarchal view is widely accepted by policy makers in Western nations. In considering physical aggression between partners, I first present the evidence, and then consider explanations based on evolutionary and social role theory, which in this case can complement one another.

\subsection{Sources of evidence}

Three meta-analyses systematically examined the evidence on sex differences in physical aggression between partners, and their consequences. The first (Archer 2000a) concerned the acts of physical aggression between partners, from self- and partner-reports and from composites of the two. Its aim was to assess whether men and women differed in the occurrence and frequency of any form of physical aggression. Most studies used the Conflict Tactics Scales (CTS; Straus 1979), which asks men and women to rate the frequency with which they have used and received various forms of behavior, including acts of physical aggression, in solving relationship conflicts. A second meta-analysis (Archer 2002) examined these acts separately, and a third (Archer 2000a) their consequences, in terms of the frequency of injuries. This was undertaken to assess whether the CTS underestimates the impact of partner violence on women because it concentrates on acts of aggression rather than their consequences.

\subsection{Acts of physical aggression and their consequences}

The mean weighted effect size for the sex difference in physical aggression, for composites of self and partner reports, was $d=-.05$, a very small, but statistically significant, effect in the female direction (Archer 2000a). Only 19 of the 82 studies involved interval-level data, although effect sizes in these were similar to those involving categorical data. The value for self-reports was slightly larger $(d=-.12)$, and from partner-reports it was near to zero $(d=-.016)$. The important conclusion is that even partner reports - those that are less likely to be subject to bias (Archer 1999) - do not show a sex difference in the male direction. For students in dating relationships, the sex difference was more in the female direction $(d=-.10 ; k=42)$ than it was in community samples $(d=-.03 ; \mathrm{k}=27)$. In two samples of women from refuges for female victims of partner violence, reporting on their own and their partner's levels of aggression, the difference was, as expected, very high in the male direction $(d=.86)$. Thus, concentrating on samples involving victims of domestic violence produces a sex difference in the male direction, which is different from that found in community samples.

The second meta-analysis (Archer 2002) examined each item of physical aggression on the CTS to answer the question of whether men were more likely than women to show more-damaging acts, and women less-damaging acts. The data (from 58 studies) were in the form of proportions of men and women showing (and/or receiving) specific acts of physical aggression. Table 4 shows the effect sizes, and the proportions of those who showed each act that were men. For self-reports, women were significantly more likely to commit most acts of physical aggression. 
Table 4. Effect sizes for sex differences in acts of physical aggression to partners

\begin{tabular}{lcc}
\hline \hline $\begin{array}{l}\text { Act of aggression towards } \\
\text { partner }\end{array}$ & Self-reports & Partner-reports \\
\hline $\begin{array}{l}\text { Threw something at } \\
\text { Pushed, grabbed or }\end{array}$ & $-.18[.37]$ & $-.08[.44]$ \\
$\quad$ shoved & $-.03[.49]$ & $.09[.54]$ \\
Slapped & $-.27[.33]$ & $-.15[.41]$ \\
Kicked, bit, or hit with fist & $-.20[.35]$ & $-.09[.44]$ \\
Hit or tried to hit with & $-.15[.36]$ & $-.08[.44]$ \\
$\quad$ something & $.03[.55]$ & $.13[.68]$ \\
Beat up & $-.003[.48]$ & $-.05[.42]$ \\
$\begin{array}{l}\text { Threatened with a knife } \\
\text { or gun }\end{array}$ & $.005[.52]$ & $.015[.53]$ \\
Used a knife or gun & & \\
\hline \hline
\end{tabular}

Figures are mean weighted $d$ values from 58 studies, with the proportion that were men given in square brackets (Archer 2002). The items are derived from the Conflict Tactics Scale (Straus 1979).

Partner reports altered these figures slightly in the male direction. However, even "beat up" showed a substantial proportion of women perpetrators (self: .32; partner: .45).

A third meta-analysis (Archer 2000a) assessed whether women sustain more injuries from a partner's physical aggression than men do (as predicted by Dobash et al. 1992). Injuries were, as expected, more common among women than men. Although in the male direction, the effect size was small $(d=.08)$. The proportion of all those injured who were women was .62 for injuries, and .65 for those requiring medical treatment (corrected for unequal sample sizes). Similar proportions were found in the 1996 British Crime Survey (Mirrlees-Black et al. 1998), again indicating a significant proportion of male injuries.

\subsection{Conclusions from the meta-analyses}

These meta-analyses clearly indicate that, in general samples, there is little difference in the proportion of each sex using any act of physical aggression. A composite of the range of acts and how often they are used also showed little sex difference. Attacks that inflicted injuries were more likely to be perpetrated by men than women, but a significant minority of injuries was caused by women. The findings are inconsistent with the assumption that partner violence only involves men's aggression, and therefore with explanations involving only patriarchal values. They are compatible with "gender-inclusive" explanations (Hamel \& Nicholls 2007; Straus 1999; Straus \& Gelles 1988) that link partner violence more with the types of psychological processes investigated by social psychologists, such as frustration, poor impulse control, personality profiles, and attachment styles, all of which apply to both women and men.

The conclusion from these studies seems to be that there are no appreciable sex differences in physical aggression to opposite-sex partners, and therefore there is no need to look for ultimate explanations or for mediators. While this may seem a reasonable conclusion, the available evidence is derived almost exclusively from people living in the late twentieth century in technologically advanced Western nations. As indicated in section 4.5, these provide an unusual sample when considered from a cross-cultural and historical perspective.

\subsection{Cross-national variability in physical aggression to partners}

The meta-analyses of physical aggression to partners are limited by the data-base from which they are derived: 72 of 80 studies used in the first one (Archer 2000a) were from the United States, and another seven were from Canada or the United Kingdom; a large number of the studies involved young dating samples from the United States. Figure 2 shows the effect sizes for sex differences in physical aggression to partners from community samples in four non-Western nations (Efoghe 1989; Kim \& Cho 1992; Kumagai \& Straus 1983), along with the value for U. S. community studies (Archer 2000a) for comparison. The weighted mean for the United States is near to zero, whereas those for the other nations are in the male direction $(d=.15$ to .30$)$.

A systematic analysis of cross-national variations, and their relation to the societal position of women in different nations, was undertaken (Archer 2006a). The Gender Empowerment Index (GEM; United Nations Development Programme Human Development Report 1997) ${ }^{7}$ was used to measure women's relative emancipation in different nations. Across 16 nations, sex differences in partner aggression were highly negatively correlated with GEM $(r=-.79)$, and also with two measures of collectivism $(r=-.87$ and -.81$)$, which are highly associated with lack of gender empowerment. Thus, the lower that women's power is in a nation, and the more collectivist the culture, the more in the male direction is the sex difference in physical aggression from community samples. Consistent with this, an analysis of partner violence in 90 societies, from the Human Relations Area Files (Levinson 1989), showed that participation in female work groups, which provide women with financial and social support, was inversely related to wife-beating $\left(\mathrm{r}_{\mathrm{s}}=-.30\right)$ and positively related to husband-beating $\left(\mathrm{r}_{\mathrm{s}}=.36\right)$.

A further analysis of the more extensive cross-national evidence on women's victimization from their partners showed that this increased as the GEM for that nation decreased, for three sets of figures: previous year $(r=-.63 ; \quad N=25)$, current relationship $(r=-.69$; $N=15)$, and lifetime $(r=-.48 ; N=40)$. Collectivism

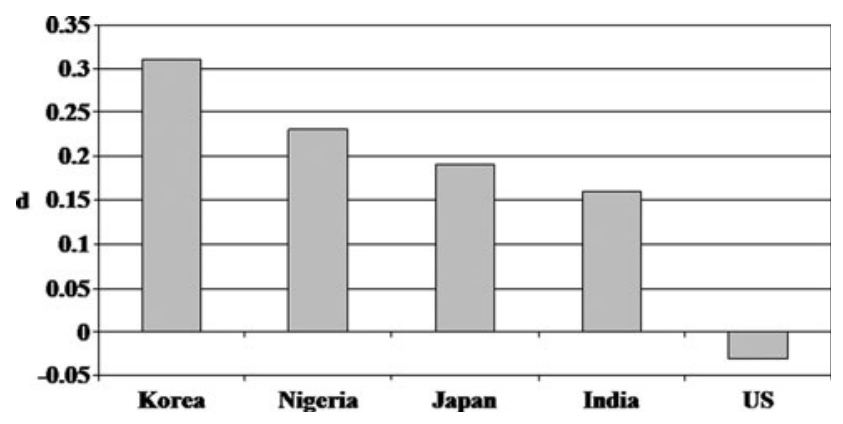

Figure 2. Effect sizes for sex differences in physical aggression to partners from studies in non-Western nations.

Source: Archer (2006a). 
showed similar correlations with victimization, which was also correlated with traditional gender attitudes and approval of a man slapping his wife (but not with national levels of violent crime). These associations suggest that gender attitudes, and attitudes towards men's violence, may be mediators between societal-level gender empowerment, and men's physical aggression to their partners. The findings support an extension of social role theory to cross-national differences in partner aggression, comparable with those found for mate choice (Eagly \& Wood 1999; Eagly et al. 2004). ${ }^{8}$

\subsection{Explaining sex differences in aggression between partners}

The cross-national analysis has considerable implications for how sex differences (or their absence) in physical aggression are explained. Rather than seeking a single explanation, as in the case of within-sex aggression, both the typical pattern and variations across nations require explanations. Many contemporary nations have relatively low gender empowerment (United Nations Development Programme Human Development Report 1997; 2005) and strong patriarchal values. This is likely to have been the usual pattern for most human societies throughout history (Betzig 1986). It is therefore likely that the typical sex difference in physical aggression to partners is that found today in low empowerment nations: that is, in the male direction. The similarity between the sexes that is found in postfeminist Western societies is likely to be attributable to historically recent changes in the position of women.

Higher male-to-female physical aggression can be explained in terms of evolutionary principles derived from sexual selection. Darwin (1871/1901) originally considered male competition and female choice, processes that occur prior to copulation. However, Trivers' (1972) emphasis on unequal parental investment (sect. 2.1.1) led to an elaboration of the consequences of males and females investing in parental care at different rates subsequent to fertilisation. Consistent with this, some theoretical treatments of sexual selection have widened its scope to include conflict between the sexes at all stages in the reproductive process (e.g., Clutton-Brock \& Parker 1995; Hosken \& Snook 2005). This has led to a number of analyses whose starting point is the conflict of interest between a male and female entering into a reproductive alliance.

Clutton-Brock and Parker (1995) set out theoretical models for the evolution of three forms of sexual coercion: forced copulation, harassment, and intimidation. Intimidation most closely fits the case of partner violence in humans. Males of a number of primate species physically punish females who refuse their mating attempts, or who consort, or mate, with more subordinate males (see Muller et al. 2007, for an empirical analysis of sexual coercion in chimpanzees). In most species where sexual coercion occurs, males are larger than females and can therefore physically dominate them. A game theory evolutionary model (Clutton-Brock \& Parker 1995) showed that where one sex (usually the male) is assumed to be more powerful than the other (the female), who is unable to offer effective retaliation, an Evolutionarily Stable Strategy (ESS; Maynard Smith 1982) is punishment by the male and learned cooperation by the female. One consequence of male intimidation in social animals is that females will seek to pair with dominant males for protection from other males. Thus, mate guarding - often viewed only from the male perspective (Shackelford \& Buss 1997; Wilson \& Daly 1992) - is likely to have benefits for the female under such circumstances.

Applying these evolutionary principles to humans, it is clear that the relatively greater size and strength of men (sect. 3.4), and their greater familiarity with physical aggression, would enable them to physically intimidate and coerce their female partners, who would as a consequence show learned cooperation. Smuts (1992) identified the following additional circumstances that would increase women's vulnerability to being beaten by their husbands: weak female alliances; lack of support from natal kin; strong male alliances; less egalitarian male relationships; and control of resources by men. Although it was argued that these circumstances would have been common in human history, as a consequence of a general pattern of patrilocal residence in human societies (Geary et al. 2003; Smuts 1992), an analysis of foragers from the standard cross-cultural sample showed a typical pattern of mutual rather than patrilocal residence (Marlowe 2004). Other evidence, based on genetic markers (e.g., DestroBisol et al. 2004), does support the view that African hunter-gathers have a history of patrilocal residence.

The situation where some men use physical aggression towards their wives is likely to be maintained unless there is a credible threat of retaliation, from the woman's male kin, or from the law. Among a sample of Mexican men and women, Figueredo et al. (2001) found that for women, density of local male kin, combined with high family support, predicted lower victimization rates. Other ways in which women's power is increased at a local level, such as female work-groups (Levinson 1989), also increase their ability to counter male domination. Increases in women's empowerment at a national level, which are reflected in gender role attitudes, and in challenges to the legitimacy of men using physical aggression against women, were also shown to be associated with lower victimization rates cross-nationally (Archer 2006a).

Both the impelling and the inhibiting forces just outlined form part of the calculus of anticipated costs and benefits of physical aggression against a partner. In societies where women have greater economic and political power, victimization rates are lessened for several reasons: Women are less economically reliant on their husbands, and can more easily escape abusive relationships; and partner violence becomes a public rather than a private issue (Felson 2002), increasing the legal and reputational costs that are imposed on violent men. These changes are accompanied by more negative attitudes towards men's physical aggression to their partners, which will have an inhibiting effect on men who might, in other circumstances, have used such aggression to control their partners' behavior. Similarly, some women will physically aggress against their partners if the fear of retaliation is lessened or is absent (Fiebert \& Gonzalez 1997).

\section{General conclusions}

I began by considering within-sex aggression. Meta-analyses of several different measurement methods showed the expected pattern of greater male than female direct 
physical, and to a lesser extent verbal, aggression when the opponent's sex was unspecified, and these findings paralleled what was found when a same-sex opponent was specified. There were no sex differences in trait anger. Girls showed more indirect aggression than boys, although by adulthood the sex difference had disappeared. This overall pattern was consistent with a sexual selection analysis involving an increasing sex difference with more costly forms of aggression.

The emergence of sex difference in physical aggression early in life, together with the very high levels of physical aggression in both sexes at age 2 years, and a decline throughout childhood, is difficult to explain in terms of gradual learning of aggressive responses through imitation and other processes. It better fits the view that physical aggression occurs as an innate pattern of behavior that is subsequently inhibited by social learning, to different extents in boys and girls. A large sex difference in damaging physical aggression occurs in young adulthood, the peak years of reproductive competition.

Mediators of the sex differences in aggression, such as the greater physical risk-taking and lesser fear of physical danger among males, have been attributed to evolved dispositions or to social roles. An interactive developmental process was suggested in which early predispositions come to manifest themselves as sex differences in social behavior through interactions with same-sex others, and these behavioral differences become conceptualized in terms of gender roles.

Variability in sex differences was explained from a sexual selection perspective in terms of alternative mating strategies and the greater variability in men's than women's physical aggression supported this. Differences in the internalization of role-related attributes were also related to variability in aggression. Different forms of context-dependent variability are predicted by sexual selection and social role theory. Findings on status and resources, and operational sex ratios, supported a sexual selection analysis. There was mixed support for social role predictions of changes in sex differences under different social role conditions, from experimental, cross-national and historical analyses.

In section 3, I have considered broader evidence for the occurrence of sexual selection in humans. Greater male than female variance in reproductive success indicates effective polygyny. Men show vocal and visual features indicative of male displays, and there is greater male than female size and strength, slower male maturation, greater male mortality, and higher male conception rates, all indicative of sexual selection. I have argued that this, in conjunction with sex differences in aggression, represents an adaptive complex arising from sexual selection.

Physical aggression to partners showed little or no sex difference, both overall and for most specific acts. The exceptions were beating up and causing injuries to a partner, although a considerable minority of men reported being beaten up or injured by a female partner. This relative equality between the sexes in acts of physical aggression was confined to nations where women have higher levels of societal power, patriarchal societies typically showing greater male than female physical aggression. The magnitude and direction of the sex difference followed a measure of societal gender empowerment and beliefs about gender roles.

For both same- and between-sex physical aggression, theoretical models derived from sexual selection can explain the basic pattern of sex differences, although the underlying principles are different. This follows not only the different evolutionary interests of the protagonists in the two cases, but also the different nature of the conflict that underlies their aggression. In the case of same-sex aggression, individuals are competing with others like themselves for mates, resources, or status that will ultimately enhance their chances of reproducing successfully. In the case of aggression to an opposite-sex partner, they are competing with individuals who have different reproductive strategies, and with whom they have to cooperate in shared parenting. In the first case, sex differences arise from the different nature of within-sex competition for the two sexes, and in the second from their evolved reproductive specializations. The evolutionary models applied to the two cases, although both characterized as "sexual selection," are very different.

Underlying all evolutionary models are the fitness costs and benefits of particular behavioral options (sect. 2.1.2). A broad principle derived from game theory models of animal fighting is that the probability of escalating a conflict to a more damaging level, for example by initiating physical aggression, is a function of the likely benefits divided by the costs (or $\mathrm{P}=\mathrm{V} / \mathrm{C}$; Maynard Smith 1982). Applied to within-sex aggression, inter-male competition can be viewed as altering the value of $\mathrm{V}$ so that males generally stand to gain more by fighting other males than females do by fighting other females. This is a consequence of males' higher reproductive rate (CluttonBrock \& Vincent 1991).

In these evolutionary models, costs and benefits refer to those imposed by natural selection. They may be reflected in basic predispositions (sect. 2.1.2), representing their incorporation into the control of behavior across a phylogenetic time-scale. This is one of three ways in which animals make adaptive responses to their environments (Waddington 1957). The other two involve developmental flexibility, enabling the individual to adapt to conditions occurring during a single lifetime, and short-term flexibility, involving learning and neuroendocrine responses (Archer 1988; 2006b). Psychological frameworks derived from learning theory (e.g., Perry et al. 1989) incorporate short-term adaptive mechanisms in the form of cost-benefit principles - for example, by viewing expectancies about outcomes as causal agents of behavior. Later social role formulations (Eagly et al. 2004; Eagly \& Wood 2006) viewed roles as establishing costs and benefits that influence behavior. Thus, for women, anticipation of fewer benefits and greater costs (in the form of negative self-evaluation and the disapproval of others) will decrease the likelihood of responding to provocation with overt aggression. Used in this sense, costs and benefits refer to those in the immediate environment as assessed by the individual. This can be fitted into Waddington's evolutionary framework as a form of short-term flexibility.

Both evolutionary (e.g., Gangestad et al. 2006a), and social role analyses (Eagly 1987; Eagly et al. 2000; 2004) emphasize variation according to the social context. Such variations in contingencies may act to accentuate, counteract, or reverse the typical sex difference. An example from section 1.8 is that when reproductive competition between women is high, their physical aggression is more common (Campbell 1995; Schuster 1983; 1985). Presumably, peer 
disapproval of direct aggression to other women is lessened, and beliefs about acceptable behavior for women are altered accordingly. In modern professional life, the costs of physically aggressing to a coworker are likely to be high, so that indirect aggression - which entails lower direct costs - will be used (Björkqvist et al. 1994; Rutter \& Hine 2005). In this context, beliefs about what is acceptable behavior for men are altered. These examples involve influences that tend to reduce the sex difference, but there are other influences, such as the culture of honor (sect. 2.8), that increase it.

Sex differences in aggression between partners can also be viewed as following the immediate costs and benefits operating in particular social environments. Here the benefits will be control of the partner's behavior, rather than resource or status acquisition as is the case for within-sex competition. The main evolutionary model covering aggression between sexual partners (Clutton-Brock \& Parker 1995) involves compliance by the physically weaker sex as a result of force by the stronger sex. This situation, which arises from sex differences in size and strength, has been viewed as forming the evolutionary origin of patriarchal beliefs (Smuts 1995), which will in turn legitimize, accentuate, and to some extent control, men's violence against their partners. The legitimacy of "wife beating" (Campbell 1992) is a feature of patriarchal societies (e.g., El-Zanty et al. 1995). Cultural attitudes associated with patriarchal values have undergone socially induced changes in Western nations, particularly over the last 40 years. These attitudinal changes have led to changes in the cost-benefit contingencies involved in partner violence. There are strong negative reputational costs attached to male violence towards women (Felson 2002), and when female victims have recourse to legal and social sanctions against violent men, female victimization decreases, and the level of male victimization increases (Archer 2006a).

Overall, the evidence indicates a different operation of evolutionary and social forces according to the sex of the opponent. Sexual selection provides the more comprehensive explanation for same-sex aggression, and a mix of evolutionary conflicts of interest and social roles for between-sex aggression. The sexual selection account presented here for same-sex aggression incorporates both consideration of context-dependent variations in behavior and the operation of social roles. In this account, the proximal causes of social roles are viewed as being derived from a complex interaction between innate dispositions, social development, and context-dependent reactions. Social roles feed back into this process, affecting the contingencies influencing behavior. They are not viewed as guiding the process, as in social role theory (Eagly et al. 2004, p. 270). They have their ultimate origins in evolutionary history, one that involves a sexually selected adaptive complex, containing psychological dispositions as well as the physical sex differences emphasized in Eagly and Wood's biosocial theory.

\section{ACKNOWLEDGMENT}

I thank Alice Eagly and Derek Heim for helpful comments on earlier drafts of this article.

Correspondence should be addressed to John Archer, School of Psychology, University of Central Lancashire, Preston, PR1 2 HE, Lancashire, UK [e-mail: jarcher@uclan.ac.uk].

\section{NOTES}

1. Based on the features they have in common, Archer and Coyne (2005) concluded that the earlier term "indirect" and the later term "relational" aggression refer to the same activities, and sex differences are similar whichever term is used. I therefore use the original term "indirect" in this article.

2. Dawkins and Carlisle (1976) pointed out that emphasis on past investment determining which sex would desert involved the "sunk cost" fallacy, the crucial variable being the replacement cost of deserting. However, past investment usually provides an indication of the future investment needed to produce offspring to the same stage as those that were lost.

3. Wood and Eagly's use of the term "bioscocial" differs from the typical use of this term, which is to refer to an approach that is specifically concerned with how genes and environment interact in development (e.g., Raine et al. 1997).

4. In these and all the studies of children mentioned in this article, play-fighting ("rough and tumble play") is excluded from consideration, and has been studied separately, since it is motivationally distinct from physical aggression (Blurton Jones 1972; Boulton 1994).

5. If it were the case that men were answering with other men in mind and women with their male partners in mind, we should expect a standardized difference for general questionnaires that is mid-way between the values where the sex of the opponent is specified. It is not. This deduction needs to be tested directly in future studies by asking respondents which sex of opponent they had in mind when completing the questionnaire.

6. In simple hunter-gatherer societies, the rates of withingroup aggression are relatively low (Knauft 1991), although rates of between-group aggression may be high (Wrangham et al. 2006).

7. GEM is a national-level variable derived from a combination of: (1) the proportion of women in managerial, administrative, professional, and technical posts; (2) their share or earned income; and (3) their parliamentary representation.

8. These analyses are now contentious since a reanalysis controlling for income, latitude, and region found that mate preferences were then unrelated to gender empowerment (Gangestad et al. 2006a). Eagly and Wood (2006) argued that by controlling for these, variables closely related to the equality of the sexes were removed. However, these variables are not conceptually related to women's empowerment, and consequently they can more reasonably be interpreted as ecological variables influencing mate choice (Gangestad et al. 2006b).

\section{Open Peer Commentary}

\section{Ultimate and proximate influences on human sex differences}

doi:10.1017/S0140525X09990483

Drew H. Bailey, Jonathan K. Oxford, and David C. Geary Department of Psychological Sciences, University of Missouri, Columbia, MO 65211.

dhbd45@mizzou.edu jkoc52@mizzou.edu GearyD@missouri.edu http://web.missouri.edu/ gearyd/

Abstract: We agree with Archer that human sex differences in aggression are well explained by sexual selection, but note that "social learning" explanations of human behaviors are not logically mutually exclusive from "evolutionary" explanations and therefore should not be framed as such. We discuss why this type of framing hinders the development of both social learning and evolutionary theories of human behavior. 
Debate regarding the origins or even existence of sex differences began with Darwin's (1871/1901) seminal contribution and continues to this day. Denials of so-called biological influences on sex differences are less common than they once were, but arguments that such influences are trivial in relation to social-psychological ones are common (Hyde 2005). Evaluations of the relative influence of these mechanisms often pit "evolutionary" against "social learning” explanations (e.g., Wood \& Eagly 2002). Archer proposes that, "the magnitude and nature of sex differences in aggression, their development, causation, and variability, can be better explained by sexual selection than by the alternative biosocial version of social role theory" (target article, Abstract; emphasis added). We argue that Archer's review, along with many previous contributions to this debate, assume, either implicitly or explicitly, that sexual selection and social learning are alternative explanations - but in fact, they are not necessarily so.

Progress in our understanding of evolutionary and social learning influences on expressed sex differences is hampered by mutually exclusive contrasts of these classes of theories. By focusing on proximate mechanisms and implicitly assuming these are alternatives to an ultimate mechanism, social learning researchers prevent themselves from integrating ultimate level influences on human behavior, including the capacity to socially learn, into their models. Understanding evolutionary influences on social learning can inform evolutionary and social learning researchers alike (e.g., Öhman \& Mineka 2001). Furthermore, evolutionary researchers who view social learning as an alternative to evolutionary theory might be missing many nuances in the ways in which evolved biases can be expressed in our species, and the evolved mechanisms that enable this variation in expression. We agree with many of Archer's concerns about the social roles model of sex differences in intrasexual aggression, and agree that sexual selection provides a very powerful and parsimonious explanation, and that the social roles model struggles on many dimensions. However, we ask Archer and others to reframe these arguments in terms of explicitly stated ultimate and proximate mechanisms. Sexual selection is necessary, in our view, for a complete understanding of the sex differences in intrasexual aggression but does not provide sufficient explanation for the variation in how men's competitive dominancestriving and behavioral aggression is expressed.

Intrasexual, male-male competition is found throughout the world, but the ways in which it is expressed can differ substantively from one culture or historical period to the next. Irons' (1979) concept of cultural success allows us to understand how ecology, cultural history, and current conditions influence how men express an evolved desire for status vis-à-vis other men. Pastoral raiders who steal another tribe's cattle to pay bride price and Wall Street raiders who seek hostile takeovers of competitor's companies may seem different on the surface, but they are not: Each of these activities is an expression of men's desire for control of the resources that affect their reproductive prospects and general well being in their culture. A Wall Street raider does not, of course, need that extra $\$ 10$ million to attract a bride or live well, but as long as there are other raiders who make more than he does, our ambitious raider will continue the struggle.

This said, we agree with Archer, that male-on-male behavioral aggression is a manifestation of our evolutionary history, and reflects a motivation to achieve social dominance and cultural status at a proximate level. But even the clearest indicators of an evolutionary history of male-male competition - the sex differences in physical size, other physical traits, and behavioral aggression - are expressed in more ways than are found in other species (Geary 1998).

Because they represent different levels of analysis, different types of data would be required to falsify hypotheses based on social learning and sexual selection. To falsify a social learning model, one would need to assess the proposed proximate mechanisms, not contrast the model with one that focuses on ultimate mechanisms. As one example, boys and girls who were not exposed to their respective "social roles" should not be as sextyped as their same-sex peers who were exposed to these roles. One type of evidence comes from children of parents who discourage sex-typing. These children have less sex-typed explicit beliefs about sex roles, in keeping with a social learning component, but have the same toy and play preferences as other children, inconsistent with a causal link between this knowledge and behavioral sex differences (Weisner \& Wilson-Mitchell 1990). Male-typical behaviors in biological males raised as girls (Colapinto 2001; Reiner \& Gearhart 2004) are especially difficult to reconcile with a strict social learning model of gender development.

These results and others (e.g., Berenbaum \& Hines 1992) suggest sex-typed activities are influenced by prenatal exposure to androgens, a proximate mechanism in the expression of sexually selected traits. Boys' attraction to karate and baseball are consistent with male-male competition, but the fact that they are culturally specific variations of one-on-one and coalitional male-male competition suggests some forms of proximate social leaning mechanisms are operating. Like Archer, we do not believe these mechanisms are the same as those identified in the social roles model. Rather, the activities that capture children's attention and that they wish to engage in, or not, are influenced by prenatal exposure to androgens, but the specifics of these activities (e.g., ice hockey) depend on exposure and the opportunity the activity affords for the expression of physical and social dominance and the formation of male coalitions (Geary et al. 2003).

In short, we believe that Archer is correct in his conclusions that male-male aggression is well explained by sexual selection and poorly explained by social roles. Our point is that by framing the argument in terms of evolutionary mechanisms versus social leaning mechanisms, Archer and many others miss the opportunity to integrate these different levels of explanation. What are the proximate attentional, cognitive, motivational, and social learning mechanisms that enable boys and men to engage in sexually selected intrasexual competition in so many creative and varied ways?

\section{Does sexual selection explain why human aggression peaks in early childhood?}

doi:10.1017/S0140525X09990471

\section{Christina Behme}

Department of Philosophy, Dalhousie University, Halifax, Nova Scotia B3H 4P9, Canada.

Christina.Behme@dal.ca

Abstract: Archer provides seemingly compelling evidence for his claim that sexual selection explains sex differences in human aggression better than social role theory. I challenge Archer's interpretation of some of this evidence. I argue that the same evidence could be used to support the claim that what has been selected for is the ability to curb aggression and discuss implications for Archer's theory.

Before turning to the main point of my commentary I want to note that Archer's definition of sexual selection as involving "the choice of members of one sex by the other, and competition by members of one sex for access to the other" (target article, sect. 2.1.1) is not uncontested. Archer does not acknowledge, let alone resolve, the controversies surrounding historic and contemporary accounts of sexual selection (e.g., Andersson 1994; Cornwell \& Perrett 2008; Cronin 1991; Darwin 1871/1901; Hubbard 1990; Johnstone 1995; Kirkpatrick \& Ryan 1991; Leonard 2005; Miller 2006; Roughgarden et al. 2006; Stamos 2008; Wade \& 
Shuster 2002; Wallace 1871; Williams 1975). Darwin held that modifications acquired through sexual selection are strongly pronounced and fully developed only at maturity. He did not attribute all differences between the sexes to sexual selection but only those differences that could not be explained based on natural selection alone (Darwin 1871/1901). Since Archer links aggression to endocrine mechanisms that also underwrite larger size and musculature in males he needs to show that these traits are not fitness-enhancing outside of the realm of reproduction. If that were the case, they (and subsequently aggression) could be explained based on natural (not sexual) selection.

If we use Archer's definition of sexual selection, several issues need to be resolved. First, Archer focuses on the differences in (physical and verbal) aggression between sexes. He provides compelling evidence that these differences arise at around 24 months and are greatest in adolescence and early adulthood. Because sex differences arise so early, Archer concludes that they cannot be based solely on cultural learning throughout childhood (sect. 2.2.1). However, he has not shown that sex differences exist already when aggressive behaviour first emerges. "Rage reactions" occur in neonates; aggressive behaviour has been documented in 4- to 6-month-old infants of both sexes (Parens 2008) and is well developed in 12-month-old infants (Holmberg 1980; Lewis et al. 1990; Sroufe 1995; Stenberg \& Campos 1990; Stenberg et al. 1983). Alink et al. (2006) did not find sex differences in aggression in 12-month-olds. For Archer's account it is important to uncover exactly when and why sex differences arise.

It is well documented that physical aggression peaks in early childhood and decreases continually. Hartup (1974) reported that 4- to 6-year-olds are more aggressive than 6- to 7-yearolds. Cummings et al. (1989) found that frequency of physical aggression, initiation of aggression, and average length of aggression periods decreased in both sexes between 2 and 5 years of age. Tremblay et al. (1999, as cited in Fig. 1 of the target article; see sect. 2.5) and Tremblay et al. (2004) found that after a peak at age 2 to 3 years, aggression decreased steadily until age 11 in both sexes. This appears to be a problem for Archer's account, because it is difficult to see how a theory of sexual selection can explain that the amount of aggressive behaviour decreases steadily throughout childhood. If aggressive behaviour (a) results in greater success in inter-male competition for mates, and/or (b) is considered by females to be an attractive characteristic of potential mates, we should expect the highest values of aggression - not only the greatest difference between the sexes - at the time when mating occurs. Instead, the peak of aggressive behaviour occurs more than 10 years before the time of mate-choice. This development is very different from that of the other sexually dimorphic traits that are part of Archer's "adaptive complex" (see sects. 3.1. to 3.4). Sex differences in these traits (e.g., body height, absolute and fat-free body mass, strength, musculature, and "aggressive display features") are usually most pronounced at the time of mating. However, we also find either a steady, "absolute" increase of these traits during childhood towards a maximum at late puberty/early adulthood (e.g., body height, body mass, strength), or a late onset (e.g., facial hair, lowering of the male voice). In no case do we have a decline in the absolute value of the trait similar to that observed in aggression. For these reasons, I suggest that it is implausible that aggression has been sexually selected for.

To maintain an evolutionary explanatory framework one might argue that females select mates that are best able to curb aggression. This move could still account for the early onset of aggression and, if we assume that aggression is underwritten by sexually dimorphic neuroendocrine mechanisms as suggested by Archer (sect. 2.1.2) and the sex differences in aggression. In addition, it could account for the decline in aggression throughout childhood. Tremblay et al. (1999) suggest that we have to learn to inhibit aggression. It is not relevant here whether this learning is based on social or evolutionary mechanisms. Both processes could contribute to sex differences in aggression. Parental feedback can impact behaviour much earlier than learning based on explicit instruction (Alink et al. 2006; Côté et al. 2006). Parents and caregivers react differently to aggressive behaviour in boys and girls, and it has been shown that this differential reaction can increase initially small differences in behaviour (Bergeron \& Schneider 2005; Fagot 1984; Fagot \& Hagan 1985; Serbin et al. 1973). Ostrov and Keating (2004) speculate that from an early age onwards, boys gravitate toward contexts in which overt aggressive tactics are more appropriate, while girls are drawn to contexts in which relational aggression is more effective. On the other hand, Chen et al. (2002) suggest that aggression can be seen as reliable indicator for poor ability to exert self-control. Hinshaw (1992) suggests that aggression is associated with academic difficulties in school-age children. Arnold (1997) and Stevenson et al. (1985) reported associations between aggression and language deficits in preschool children.

Finally, on Archer's account, women would face a conflict in mate choice. On the one hand, they would be attracted to aggressive males. On the other hand, they would be interested in a longterm relationship with a provider. It seems that females who resolve this conflict by choosing a mate who is not likely to harm them or their offspring will leave more offspring than those who choose an overly aggressive mate. This would lead to selection against aggression.

Much work remains to be done before we can understand the evolutionary history of aggression. As a longtime researcher of the field aptly put it: "We stand at the edge of the nest, then, regarding what we know about the development of aggression" (Hartup 2005, p. 19).

\section{Dominating versus eliminating the competition: Sex differences in human intrasexual aggression}

\author{
doi:10.1017/S0140525X0999046X
}

\section{Joyce F. Benenson}

Department of Psychology, Emmanuel College, Boston, MA 02115; Department of Biological Anthropology, Harvard University, Cambridge, MA 02138.

\section{Joyce.Benenson@gmail.com}

Abstract: Archer presents a traditional view of intrasexual competition. Knowledge of a species' social structure provides a more complete picture. Human males compete against individuals with whom they may cooperate later in inter-group aggression. By contrast, females compete against individuals for a mate's continued support. Females' aggression may aim at eliminating the competition, whereas males simply may attempt to dominate others.

Archer argues that across most species, males' greater intrasexual competition for mates accounts for their higher rates of aggression, with variation in paternal care elucidating the magnitude of the sex difference. Defining intrasexual competition as including not only mating contests but also breeding competition, however, provides a broader perspective (Clutton-Brock 2007; 2009). Breeding competition may include contests over food, territory, helpers, protection, and status, as well as other factors that enhance reproductive success within a particular ecology. A species' social structure determines in part how these challenges are confronted, through delineating patterns of same-sex associations, asymmetries in sex-biased investment in both kin and non-kin, and functions of same-sex alliances and coalitions (Wrangham 1987). To understand more completely human sex differences in patterns of aggression, Archer could reach 
beyond consideration of the benefits and costs of physical fighting to incorporate an understanding of a species' social structure.

Humans segregate themselves by sex early in the juvenile period (Maccoby 1988). Across varied cultures beginning in middle childhood and continuing into adulthood, human males interact in large, loosely structured, interconnected same-sex groups, whereas human females interact with one same-sex individual at a time (Benenson et al. 1997; Cairns et al. 1998; Markovits et al. 2001; 2006; Savin-Williams 1980). Additionally, human males invest more than females in, and exhibit more tolerance towards, same-sex peers (Benenson et al. 1998; 2008b; 2009). Sex differences in group versus individual investment extend to sexual partners. Like polygynously and monogamously mated primates, human females typically form a long-term bond to one sexual partner, whereas human males more frequently form bonds with multiple short- and longterm sexual partners.

Unique facets of humans' social structure should influence Archer's conclusions regarding sex differences in aggression. More than in most other species, human females rely heavily on the investment of one mate to provision and protect themselves and their offspring (Lancaster \& Lancaster 1983). Consequently, in contrast to other species, human females invest more in a mate to the exclusion of other individuals.

Likewise, unlike males in virtually all primate species (with the exception of chimpanzees, Pan troglodytes), human males routinely engage in lethal coalitionary inter-group contests (LeBlanc \& Register 2003; Wrangham 1999). Success in these contests heavily influences the survival and reproductive fitness of the entire community. Like male chimpanzees, human males therefore must balance intra-group competition for status and mates with cooperation with these same competitors during intergroup contests. Heavy investment in male peers combined with high tolerance for transgressions likely produce rapid reconciliation of within group conflicts during inter-group contests.

By their nature, groups enhance competitive and aggressive behaviors, whereas one-on-one competition disguises competition with more placating behaviors, including signals of anxiety and depression - for both sexes (Bales \& Borgatta 1955; Benenson et al. 2001; 2002). Groups enhance competition because fewer resources are available per individual and many individuals vie for rank. Because human males must maintain a group's integrity given the perpetual threat of inter-group contests, however, group members likely dampen competitions' adverse effects by providing mediators, allies, and alternate partners, and promoting loyalty to the larger group. A human male's goal in intrasexual competition consequently becomes to dominate other individuals within the group without harming the group's integrity. Within-group fighting then may be less important than between-group fighting in explaining sex differences.

Human females' competition occurs within a different structure. Isolated one-on-one competition without the support that a group provides jeopardizes the relationship's survival. Human females' same-sex dyadic relationships endure for shorter periods than those of males, most likely because of their lesser ability to resolve conflicts (Benenson \& Christakos 2003).

A human female must compete, however, not only to initiate a long-term bond with a high status mate who can enhance the survival and status of her offspring, but also to maintain her mate's loyalty. She must fend off competitors for her mate's resources and protection, or in the case of polygynous unions, for a greater share of her mate's investment.

Social exclusion of competitors provides the perfect mechanism. Because females' same-sex relationships by their nature are not interconnected or group-based, exclusion of another female can occur seamlessly. Feshbach showed that compared to their male peers, females in both early childhood (Feshbach 1969) and adolescence (Feshbach \& Sones 1971) were less welcoming to a same-sex newcomer. Likewise, in an experiment with limited resources, 4-year-old female triads were more likely than male triads to exclude the one child who obtained a resource, whereas males competed individually for the resource while maintaining the integrity of the trio (Benenson et al. 2008a). Whereas Archer, using Campbell's (1999) analysis, emphasizes that females gain less and lose more from overt fighting, he neglects to consider that females also may benefit far more than males from aggressively excluding one another.

Smuts (1987a) argues that across primate species, males generally engage in sporadic but intense bouts of aggression for mates, whereas females engage in more chronic but low-grade aggression to attain resources. In humans, males compete for status and mates but they frequently retain these mates for long periods. The premium placed on virginal status and ensured paternity also means that once a male has successfully dominated his competitor to win a virginal female, losers will be less interested in the winner's wives. Further, male winners and losers may serve as future partners in cooperative group endeavors, including lethal coalitionary inter-group aggression. Human females, by contrast, continually compete to initiate and maintain long-term bonds with a mate who can provide prolonged aid. Eliminating competitors provides continuous benefits.

Archer's perspective that sex differences in aggression result from human males' confronting greater intrasexual competition for mates neglects the unique breeding challenges of each sex that human social structure likely evolved to satisfy.

\section{Sex differences in the developmental antecedents of aggression}

\author{
doi:10.1017/S0140525X0900140X
}

\section{Joseph M. Boden}

Christchurch Health and Development Study, University of Otago, Christchurch School of Medicine and Health Sciences, PO Box 4345, Christchurch,

New Zealand.

\section{joseph.boden@otago.ac.nz}

http://www.chmeds.ac.nz/research/chds/index.htm

Abstract: Archer examines sex differences in aggression, and argues that these differences may be better explained by sexual selection theory than by social role theory. This commentary examines sex differences in the developmental antecedents of aggression and violence, and presents a preliminary framework for examining whether the observed sex differences amongst these developmental antecedents can also be accounted for by sexual selection theory.

The target article examines sex differences in aggression, arguing that differences in aggressive behaviour may be better explained by sexual selection theory rather than social role theory. In this commentary I examine the related question of sex differences in the developmental antecedents of aggression, and show that these too may be better explained by sexual selection theory, rather than social role theory.

Archer argues that the magnitude and nature of sex differences in aggression, which he defines as differences in aggression between same-sex individuals as well as between opposite-sex individuals, are better explained in terms of sexual selection than of social role. One of the key underpinnings of this argument is that evidence concerning the development of aggression suggests that physical aggression emerges early in life and tends to decline thereafter, suggesting that aggression is not a learned response.

The developmental perspective on aggression has also underpinned studies examining the causal influence of early experience on later aggression. Several studies have examined the extent to which certain developmental factors, such as family functioning, socio-economic conditions, exposure to abuse, and other factors can account for aggression and violence later in life (Daigle et al. 
2007; Fergusson et al. 2008; Howells \& Rosenbaum 2008). One of the key findings common to these studies is that there may be reliable sex differences in the extent to which certain environmental or behavioural factors may be related to later aggression. While this is a somewhat different issue than that addressed by Archer, an examination of the pattern of sex differences in the developmental antecedents of aggression shows that these differences can also be better explained by sexual selection theory than by social role theory.

Although a wide range of studies have examined the developmental processes that predispose individuals to aggression and violence (for reviews see, e.g., Emery \& Billings-Laumann 1998; Loeber \& Hay 1997; Tolan et al. 2006), relatively few studies have identified sex differences in the extent to which certain risk factors may have differential effects on males and females in terms of predicting later violence. One such study was conducted by Fergusson et al. (2008), using data from a longitudinal birth cohort. These researchers found that several factors predicted both perpetration of and victimization by intimate partner violence (IPV) in adulthood, including childhood conduct problems, exposure to family adversity, abuse exposure, and adolescent alcohol abuse/dependence. Importantly, however, they found that exposure to family adversity was more strongly predictive of later IPV involvement for males, whereas childhood conduct problems were more strongly predictive of later IPV for females. Fergusson et al. concluded that the data suggested a varied developmental pathway to IPV for males and females, although the precise mechanisms behind this pathway were unclear. Comparable findings were reported by Howells and Rosenbaum (2008) and by Daigle et al. (2007).

Social role theory (e.g., Eagly 1997; Eagly \& Steffen 1986) would predict that sex differences in the developmental antecedents of aggression and violence should reflect the differential sex-role socialization experienced by males and females. For example, under such an explanation we will expect males to be more influenced by exposure to violence or by affiliation with violent and aggressive peers (both features of the male sex role under social role theory). On the other hand, on the assumption that the socialization of females tends to move individuals away from violence and aggression, it may be expected that females will be more influenced by the weakening of social bonds via family dysfunction.

The data on sex differences in the developmental antecedents of aggression do not seem to be congruent with this position, however. For example, Fergusson et al. (2008) found that a broad measure of family dysfunction predicted later IPV for males more strongly than females. This finding suggests that the weakening of social bonds caused by dysfunctional family processes increases the risks of violence among males relative to females, counter to what would be expected under social role theory. Furthermore, conduct-disordered behaviour in childhood predicts adult IPV involvement more strongly for females than males, suggesting that there are lower levels of continuity of aggressive behaviour across the lifespan among males than females, again counter to what would be expected under social role theory.

The question then arises: Can sexual selection theory better explain the sex differences observed in the developmental antecedents of violence and aggression? Archer argues that sexual selection theory would view variability in aggression as reflecting resources important for reproduction. In the cohort studied by Fergusson et al. (2008), males at greater risk of later aggression were more likely to have come from dysfunctional homes in which they were at greater risk of exposure to a wide range of environmental stressors, including material deprivation. It could therefore be argued that exposure to family adversity increases violence and aggression in males by making salient resource limitations, engaging adaptive modules that serve the purpose of increasing access to resources (via aggression). Furthermore, in the study by Fergusson et al. males were less likely than females to show continuity of aggression, in terms of childhood conduct disorder being linked to adult IPV involvement. Again, this may be linked to Archer's general argument that, under sexual selection theory, there should be greater variability among males than females in terms of the effect of local environmental conditions. If we extend "local environmental conditions" to include environmental conditions across the lifespan of the individual, the greater discontinuity in males relative to females might reflect adaptation to variable environmental influences on aggressive behaviour.

In summary, I agree with Archer that an initial examination of the evidence pertaining to sex differences in the developmental antecedents of aggression appears to support the notion that aggression is primarily a product of sexual selection, rather than social role. Further research is needed to shed light on this question.

\section{Sex differences in aggression: Origins and implications for sexual integration of combat forces}

\author{
doi:10.1017/S0140525X09990458
}

\section{Kingsley R. Browne \\ Wayne State University Law School, Detroit, MI 48202. kingsley.browne@wayne.edu \\ http://faculty.law.wayne. edu/browne/index.htm}

\begin{abstract}
Sex differences in aggressive and risk-taking behaviors have practical implications for sexual integration of military combat units. The social-role theory implies that female soldiers will adapt to their role and display the same aggressive and risk-taking propensities as their male comrades. If sex differences reflect evolved propensities, however, adoption of the soldier's role is unlikely to eliminate those differences.
\end{abstract}

The choice between the evolutionary approach advocated in the target article and the biosocial approach that it criticizes, is an important one that is of more than academic interest. In fact, it has direct application to a critical policy choice facing modern governments: whether women should be integrated into military combat units.

A variety of sex differences are relevant to the integration question. Most obvious are differences in physical attributes - such as size, strength, speed, and endurance - which overwhelming evidence shows are primarily a consequence of sex hormones (Cheuvront et al. 2002). Differences in intragroup interactions between all-male and sexually integrated groups also bear on the question of integration, as issues of group cohesion, sexual relationships, male protectiveness toward women, among others, are of central importance (Browne 2001; 2007). Of specific relevance to the subject of the target article are sex differences in aggressiveness and physical risk-taking.

The biosocial theory attributes sex differences in aggressiveness and risk-taking to "the distribution of women and men into different specific roles in societies” (Becker \& Eagly 2004). Characteristics such as a propensity to engage in risky behavior are imputed to men because men are more likely to occupy roles requiring such action due to their greater physical prowess and the restrictions that childbearing places on the activities of women.

A critical flaw in the biosocial theory is the lack of curiosity it exhibits over the origins of physical sex differences. The theory assumes that the evolutionary forces that left their imprint on human bodies had no similar effect on human minds, an assumption that is untenable if one reflects on where these physical differences came from. Because these differences are consistent with a common mammalian pattern - according to which sexual 
size dimorphism appears to be an evolved consequence of malemale competition that has behavioral correlates - the principle of parsimony would, at least as a first cut, suggest common origins. Needless to say, a social-role explanation for behavioral sex differences throughout the mammal world is difficult to credit.

If men exhibit greater aggressiveness and risk-taking simply because they have been placed in roles that demand, or at least reward, these attributes, then one might expect that placing women into the role of combat soldier will cause them to exhibit the same kinds of aggressiveness and risk-taking as male soldiers. Because women have not widely served in combat, there is no clear empirical evidence directly on point. However, there are some data to show that even female soldiers respond differently to combat risks than men do.

A number of press reports from Iraq suggest that female soldiers are at least perceived as less aggressive than their male comrades. One story (in The Times of London), for example, described a female U. S. Army helicopter pilot who requested that the reporter use the term "neutralise," rather than "kill," because she did not want to create an erroneous impression that soldiers enjoyed killing. The reporter noted that "her sensitivity stands out in an army in which male soldiers talk of 'smoking,' 'wasting,' or 'whacking' the enemy" (Meo 2006, p. 46). Another newspaper story (in the Washington Post) profiled a different female helicopter pilot who objected to the bellicosity of her male comrades: "Everyone was like, 'Yeah, get them' and I was having trouble with that really aggressive attitude" (Tyson 2005, p. A-1). According to a Chicago Sun-Times article, a female National Guard gunner noted that women do not fire their weapons as much as men do because of their greater caution. She continued:

Men are more aggressive and trigger-happy. We have a lot of younger guys - 18-, 19-year old guys - who can't wait to get their first kill. Women don't look at death that way. We would rather solve the situation. If somebody has to die, then nobody really wins. (Reed 2005, p. 4)

I concede these are anecdotal accounts and are not necessarily representative. However, there is little reason to believe that they present a false picture.

Mere presence in a war zone is considerably more stressful to women than to men. Among male and female soldiers serving in non-combat positions during the Gulf War, women reported experiencing significantly more psychological stress than men, especially stress in anticipation of combat (Rosen et al. 1999). Reports from Iraq indicate that women are suffering posttraumatic stress disorder at approximately twice the rate of men, and suffering from more severe forms, despite the fact that women are exposed to considerably less combat danger (Scharnberg 2005, p. C-1). These reports are consistent with the view articulated in the target article that women exhibit higher fear levels than men.

The target article notes that studies typically find no sex difference in anger. However, there are sex differences in the correlates of anger that are likely to facilitate physically aggressive behavior in men and to be of importance in combat. Fessler et al. (2004) have shown that anger increases risk-taking among men but does not do so for women. On the other hand, disgust inhibits risk-taking among women but not among men. Because the battlefield provides ample opportunity for both anger and disgust to operate, patterned differences between men and women in combat performance are predictable.

Average differences between the sexes are not necessarily a reason to exclude women from combat. In theory, soldiers could be selected based upon their individual attributes, including aggressiveness and risk-taking. One problem is that, as the target article notes, sex differences in naturalistic settings are generally greater than those exhibited in labs and on paperand-pencil tests. Moreover, unlike strength, which can be easily and cheaply screened for, future courage under fire cannot be readily measured. A consistent theme in the combat-behavior literature is that one never knows who is going to be an effective soldier until the shooting starts, and the identity of the good fighters often turns out to be a surprise (Braun et al. 1991; Browne 2007, p. 111).

The question of whether to integrate the sexes in combat forces is an important one, with many lives potentially hanging in the balance. Knowledge of the origins of sex differences in aggression and risk-taking does not by itself determine appropriate military manpower policy. However, any policy adopted is more likely to succeed if it is grounded in accurate factual assumptions.

\section{The multiple adaptive problems solved by human aggression}

\author{
doi:10.1017/S0140525X09990343
}

\author{
David M. Buss \\ Department of Psychology, A8000, University of Texas, Austin, TX 78712. \\ dbuss@psy.utexas.edu \\ www.davidbuss.com
}

Abstract: Human psychology contains adaptations to deploy aggression as one solution to many distinct adaptive problems. These include expropriating resources, defending against incursions, establishing encroachment-deterring reputations, inflicting costs on rivals, ascending dominance hierarchies, dissuading partner defection, eliminating fitnessdraining offspring, and obtaining new mates. Aggression is not a singular strategy. Comprehensive theories must identify the "design features" of multiple adaptations for aggression.

Archer makes a compelling case - conceptually and empirically - that many patterns of human sex differences in aggression, and adaptations that contributed to an aggressive strategy, have arisen by the evolutionary process of sexual selection. Alternative theories that assume a sexually monomorphic evolved mind, such as social role theory, are implausible on conceptual grounds and have so much empirical evidence against them that they can safely be consigned to a footnote in the history of psychology. I suggest that the case for sexual selection in explaining sex-differentiated patterns of aggression is even stronger than that presented in Archer's target article, and that progress in understanding human aggression will be advanced by a more detailed consideration of the adaptive problems solved by implementation of an aggressive strategy.

Hypotheses about adaptive problems solved by aggression lead to predictions about psychological and behavioral "design features" of adaptations for aggression. Archer alludes to some of the broad classes when he suggests that contingent use of aggression evolved to solve adaptive problems of competing for mates, resources, and status; and he details some of the attendant anatomical and physiological features that accompany adaptations for aggression. The specific ways in which competitions occur provide a deeper understanding of aggression, as well as powerful evidence supporting Archer's overarching claim.

Many theorists have proposed that aggression evolved as a context-contingent solution to a host of adaptive problems, including: appropriating the resources of others; reacquiring resources previously appropriated by competitors; preemptively defending against attack; establishing a reputation that deters aggression from others; inflicting costs on intrasexual rivals that damages their ability to retaliate; ascending dominance hierarchies; dissuading romantic partners from infidelity; aggressive stalking to acquire new mates or regain former mates; eliminating fitness-draining offspring; and obtaining sexual access to the otherwise inaccessible (Buss 2005; Buss \& Duntley 2006; 2008; 
Buss \& Shackelford 1997b; Campbell 1995; 2002; Duntley \& Shackelford 2008; Smith 2007; van der Dennen 1995). Examination of a few of these reveals details about adaptations for aggression, and renders the case for sexual selection origins stronger.

Consider war, a particularly dramatic a form of aggression. Mythology aside, there exists not a single case in which women formed same-sex coalitions to kill other female coalitions for the purpose of purloining resources, territory, and mates. Yet history is replete with evidence that men routinely wage war for precisely these purposes (Buss 2005; Chagnon 1983; Tooby \& Cosmides 1988; Smith 2007; van der Dennen 1995). Identifying adaptations for this specific form of aggression reveals additional evidence in support of the sexual selection origins. Among the Yanomamo of Venezuela, unokai men (those who have killed) have more wives and children than those who have not killed (Chagnon 1988). The paleontological evidence brims with findings of male skulls and skeletons, with injuries corresponding in size and shape to weaponry existing at the time, and an otherwise inexplicable dearth of female skulls and skeletons (Grauer \& StuartMacadam 1998). DNA studies of genetic signatures suggest that warriors who vanquished other groups of men sired many progeny (Zerjal et al. 2003), pointing directly to the sexually selective benefits of aggression.

Ascending status hierarchies is almost certainly one evolved function of physical aggression, as is using aggression to maintain positions attained. History is replete with influential leaders such as Joseph Stalin of Russia, Pol Pot of Cambodia, Saddam Hussein of Iraq, Idi Amin of Uganda, François Duvalier of Haiti, Benito Mussolini of Italy, Ion Antonescue of Romania, Mao Zedong of China, Kim Il Sung of North Korea, Ferdinand Marcos of the Philippines, Slobodan Milosevic of the former Serbia, Ne Win of Burma, and Pablo Escobar of Colombia - all of whom murdered to get to the top, and continued to murder to quash competitors in order to maintain their status positions (Buss 2005). Most turned their positions of power into mating opportunities, again supporting a sexual selection explanation.

Although these examples might imply that men have a monopoly on aggression, examination of other adaptive problems and their evolved solutions suggest a more nuanced depiction. Evidence points to differently designed infanticidal adaptations in women and men. Women are more inclined to kill their infant when they have many years of future reproduction ahead of them, or when the infant lacks an investing father or is congenitally deformed (Daly \& Wilson 1988). Men are more inclined to kill infants when there is suspicion of knowledge of a lack of paternity (Daly \& Wilson 1988).

Less gruesome forms of aggression, such as derogation of competitors, show sex-differentiated design features. Women derogate rivals along the dimensions of sexual promiscuity, sexual fidelity, and physical appearance (Buss \& Dedden 1990; Campbell 2002). Men are more likely to derogate rivals along the dimensions of resources, future resource trajectories, physical strength, and athletic prowess (Buss \& Dedden 1990; Schmitt \& Buss 1996). These findings suggest a complex way in which sexual selection has influenced human aggression. The domains of intrasexual competition (one component of sexual selection) are dictated by the mate preferences of the opposite sex (the second component of sexual selection) (Buss 1988b).

Sexual selection, in short, provides a powerful overarching theory of the origins of human aggression, but not solely in explaining the broadly based sex differences in physical aggression. Sexual selection theory also explains many forms of female aggression. Perhaps most important, identifying with greater specificity the adaptive problems for which aggression evolved as one context-contingent solution provides a key to future scientific advances. In this sense, aggression is not singular in nature, but rather is an overarching term for a collection of context-specific cost-inflicting strategies.

\section{What kind of selection?}

\author{
doi:10.1017/S0140525X09990446
}

\section{Anne Campbell \\ Psychology Department, Durham University, Durham DH1 3LE, United Kingdom. \\ a.c.campbell@durham.ac.uk} Abstract: Supporting a mediating role for fear in inhibiting female
aggression, a recent study shows that aversion to "risky" impulsivity
completely mediates the sex difference in direct aggression but not in
angry acts where dangerous retaliation is unlikely. A more inclusive use
of the term "sexual selection" to encompass reproductive advantage
would recognise females crucial role in nurturing and protecting
offspring.

A perplexing paradox for aggression researchers is the marked sex difference in same-sex aggression and its absence in partner-directed aggression. Archer is well placed to take on the explanatory challenge, given his impressive output of metaanalyses in this area.

In discussing fear and risk-taking as candidate mediators of the sex difference in aggression, Archer notes that "there are currently no direct tests of these." However, a colleague and I recently conducted such a study (Campbell \& Muncer, in press). We developed a scale of "risky impulsivity" - defined as a tendency to act spontaneously and without deliberation, where the act has potentially dangerous consequences. We purposely omitted any reference to hostile interchanges. We also measured direct physical and verbal aggression, both of which involve potentially injurious retaliation by the victim, and two forms of anger expression that do not involve confrontation and are therefore unlikely to excite retaliation. One form was what we called "defusing" anger (taking actions that reduce anger intensity, such as retreating from the scene or discussing the incident with a third party) and the other was "explosive" anger (discharging acute physical or verbal anger when alone, e.g., by hitting walls or shouting). Sex differences in physical and verbal aggression were eliminated when risky impulsivity was controlled. This complete mediation of sex differences was restricted to the two direct forms of aggression, those carrying the risk of retaliation.

Archer uses the term "sexual selection" to describe the evolution of sex-differentiated traits, but there has recently been heated controversy about the scope and interpretation of this term (Kavanagh 2006; Roughgarden et al. 2006). Darwin himself $(1871 / 2004$, p. 245) left the door open to such debate when, with disarming honesty, he acknowledged that, "in most cases ... it is impossible to distinguish between the effects of natural and sexual selection."

Evolutionary psychologists have mainly restricted "sexual selection" to intrasexual or intersexual competition for mating opportunities. When male variance in reproductive success exceeds that of females, there is effective polygyny and intensified male competition. Daly and Wilson's (1988) account of sex differences in aggression clearly identifies these mating opportunities as the driver of male competition and, in extremis, aggression. Under monogamy, there is two-way selection and researchers have now begun to address women's intersexual competition for mates. One popular topic is male preference for female body shape, with debate focusing on the relative importance of waist-to-hip ratio and body mass index, and the universality of these male preferences (Tovee \& Cornelissen 1999; Yu \& Shepard 1999). 
But what if female waist-hip ratio evolved, not with men's preferences, but with women's reproductive success, in mind? Bipedal locomotion, combined with pregnancy and infant carrying, meant that a lower relative centre of body mass increased women's stability and this shift corresponds with a lower waist-hip ratio (Pawlowski \& Grabarczyk 2003). Or a lower waist-hip ratio may have resulted from foetal developmental demands: The supply of long-chain polyunsaturated fatty acids needed for neurodevelopment is optimised where the mother's lower body fat exceeds upper body fat. Waisthip ratio, a proxy for this fat distribution, is positively associated with children's cognitive test scores (Lassek \& Gaulin 2008). This is not to deny men's preference for female body shapes but to suggest that the selective advantage for women was not obtaining a better mate but producing more surviving, high-quality children.

This suggests a second, more inclusive interpretation of sexual selection - "the advantage which certain individuals have over others of the same sex and species solely in respect of reproduction" (Darwin 1871/2004, p. 243). Because Darwin focused chiefly on males, with their typically lower parental investment, his notion of reproduction was largely restricted to mating opportunities. Yet, as $\operatorname{Hrdy}(1999$, p. 81) emphasises, "Unless mating results in the production of offspring who themselves survive infancy and the juvenile years and position themselves so as to reproduce, sex is only so much sound and undulation signifying nothing." And in the vast majority of mammals, mothers take this responsibility. It might therefore be argued that any female trait that confers an advantage over competitors in reproduction (in this broader sense beyond mate competition) should be considered a sexually selected trait. In my own proposal (Campbell 1999; 2002) for a psychological mediator of sex differences in aggression, this would include a lower threshold for experiencing fear.

In summarising my proposal, Archer accepts that it derives from unequal parental investment but describes it as an "alternative to the sexual selection view" of Daly and Wilson, thus implying the action of natural selection. And Darwin might agree with him:

When ... the two sexes differ in structure in relation to different habits of life, they have no doubt been modified through natural selection, and by inheritance, limited to one and the same sex ... those individuals which generated or nourished their offspring best, would leave, ceteris paribus, the greatest number to inherit their superiority. (Darwin 1871/2004, p. 243)

Certainly from the infant's viewpoint, maternal care is about its own survival and hence about natural selection. But from the mother's viewpoint, her care is about increasing her reproductive success relative to her competitors.

So we can see heightened female fear in the service of offspring survival as a sexually selected trait defined in this more inclusive sense. But if sexual selection refers narrowly to competition for copulations it will predominantly apply to males. This means that traits that increase competitive ability are more likely to be attributed to males than females (Clutton-Brock 2007), and this narrow usage devalues women's parenting effort, which is so crucial to infant survival and female reproductive success. Carranza (2009, p. 750) suggests the term "sex dependent selection" to capture "those natural selection forces that operate differently in males and females because of the different reproductive strategies of the two sexes." Specifically, fear evolved in both sexes under natural selection but was hyper-selected in females because of its association with increased reproductive success in females but not males. In that sense, men's heightened competitive risk-taking and women's lower threshold for fear are both examples of sexdependent selection.

\section{Sex differences in aggression: What does evolutionary theory predict?}

\author{
doi:10.1017/S0140525X09990318
}

\section{Elizabeth Cashdan}

Department of Anthropology, University of Utah, Salt Lake City, UT 84112-0060.

cashdan@anthro.utah.edu

http://www.anthro.utah.edu/people/faculty/elizabeth-cashdan.html

Abstract: The target article claims that evolutionary theory predicts the emergence of sex differences in aggression in early childhood, and that there will be no sex difference in anger. It also finds an absence of sex differences in spousal abuse in Western societies. All three are puzzling from an evolutionary perspective and warrant further discussion.

I agree with Archer that "Social roles ... have their ultimate origins in evolutionary history" (target article, sect. 5, last para.) and think that the difference in levels of explanation between evolutionary theory and social role theory is responsible for some of the confusion surrounding this debate. Archer's review of these theories as they apply to sex differences in same-sex aggression is very helpful, but a few of the evolutionary predictions raise additional questions.

Most of Archer's evolutionary predictions regarding same-sex conflict are clear, but two are puzzling. One is the claim that evolutionary theory predicts the early emergence of sex differences in direct aggression. If this is a sexually selected trait, why should it appear before it is needed in mating competition? Most sexually selected traits appear at puberty, so an additional argument is required to support this prediction.

Also puzzling is Archer's assertion that evolutionary theory would not predict a sex difference in anger. Emotions motivate behavior and are affected by selection only if they affect behavior. If theory predicts a sex difference in aggressive behavior, why would it not also predict a sex difference in the emotion that motivates it?

The answer may help us understand the evolutionary reasons for greater male same-sex aggression. As Archer notes, two arguments have been proposed: (1) greater benefit to males because of greater variance in male reproductive success (the usual argument), and (2) greater cost to females, due to their greater parental investment (Campbell 1999). If the first of these is driving sex differences in aggression, we should expect reduced anger in women, to motivate their less intense aggressive competition. The second argument, in contrast, would predict equally intense competition but would temper anger with fear, thereby leading to less costly, but not less emotionally-intense, forms of aggression. This seems more consistent with the data showing that women and men experience similar degrees of anger, although they may express it differently.

My chief concern with this otherwise valuable target article lies in its treatment of partner violence. Evolutionary theory provides a robust explanation for the finding that males are more likely to control sexual access to females than the converse, and often use aggression to enforce it. In view of this, Archer's claim that there is no sex difference in spousal abuse in Western nations is surprising and deserves another look. The claim of sexual symmetry ignores much contradictory evidence, ignores sex differences in motive, and relies heavily on studies using the problematic (Dobosh et al. 1992) Conflict Tactics Scale (CTS). Johnson (2006) has shown that violence involving proprietariness and control ("intimate terrorism") is heavily male-biased, unlike the disputes picked up by the CTS, which arise chiefly from conflicts of daily life ("situational couple violence"). The former also causes far more harm, both physically and psychologically, than more sexually symmetrical altercations (Johnson \& Leone 2005). Evolutionary theory that addresses male sexual proprietariness and concern over cuckoldry provides a phylogenetically broad explanation for this more serious type of male-biased 
violence, and leads to predictions that distinguish it from violence arising from conflicts of interest in other domains (Daly \& Wilson 1988; Wilson \& Daly 1996).

\section{Differentiating defensive and predatory aggression: Neuropsychological systems and personality in sex differences}

\section{doi:10.1017/S0140525X09990434}

\section{Philip J. Corr ${ }^{a}$ and Adam M. Perkins ${ }^{b}$ \\ ${ }^{a}$ School of Social Work and Psychology, Faculty of Social Sciences, University of East Anglia, Norwich NR4 7TJ, United Kingdom; ${ }^{\mathrm{b}}$ Department of Psychology, School of Human Sciences, Swansea University, Singleton Park, Swansea SA2 8PP, United Kingdom. \\ Philip.corr@btopenworld.com \\ 342095@swansea.ac.uk}

Abstract: We draw a distinction between defensive and predatory forms of aggression, and how these forms relate to basic neuropsychological systems, especially the Fight-Flight-Freeze-System (FFFS; putatively related to defensive aggression), and the Behavioural Approach System (BAS; putatively related to predatory aggression). These systems may help further to account for proximal brain processes and personality influences in the context of sex differences.

1. Behaviour as a product of evolutionary processes. Twentiethcentury psychologists devoted considerable attention to the hypothesis that behaviour is learnt, and tended to downplay, or even completely deny, the possibility that behaviour has a heritable basis (e.g., Watson 1919). A plethora of evidence, from across mammalian species, challenges this viewpoint. For example, rodents innately display species-typical defensive behaviour when exposed to a predator for the first time (Blanchard 1997); and, in humans, studies of twins have revealed that a wide range of behavioural traits are substantially heritable (e.g., Plomin et al. 2001).

One by-product of the emerging consensus that mammalian behaviour patterns are partly innate is a growing interest in explanations as to the mechanism by which behaviour becomes coded in the genome. By far the most prominent theory is that innate behaviours, such as aggression, evolved by similar processes to those that are widely accepted to have shaped anatomical features (Darwin 1859/1911). Following Darwin, Archer presents a detailed argument that human sex differences in aggression evolved primarily as a result of selection pressure that placed less aggressive males at a competitive disadvantage in the struggle for mating opportunities.

Archer is careful to note, however, that the greater propensity to aggressive behaviour in males is limited to attacks on male competitors. In situations where violence occurs between heterosexual couples or in situations of provocation, Archer cites studies that show males and females display similar levels of aggression (e.g., Archer 2000a; Bettencourt \& Kernahan 1997). We argue that Archer's already convincing argument could be further strengthened by supplementation with certain principles drawn from the work of Jeffrey Gray on defensive/ predatory behaviours and personality (Gray \& McNaughton 2000; updated by McNaughton \& Corr 2004; 2008; for a summary, see Corr 2008).

2. Reinforcing stimuli as mediators of aggression. Gray's approach postulates that the various classes of emotional behaviour displayed by animals are dependent upon the interplay of three major neuropsychological systems. Threat stimuli activate a Fight-Flight-Freeze-System (FFFS), leading to avoidance/escape and the emotions of fear and panic/rage (depending on the level of threat perceived) - this system is specifically related to defensive aggression. Reward stimuli activate a Behavioural Approach System (BAS), leading to approach behaviour and the emotion of hopeful anticipation, optimism, and so forth - this system may be related to predatory aggression (see below). Ambiguous and conflicting stimuli (e.g., approach-avoidance conflict) activate a Behavioural Inhibition System (BIS), leading to risk assessing cautious behaviour and the emotion of anxiety (reflecting the apprehension that something bad may occur at any moment) - activation of this system should be expected to inhibit aggression. Applied to personality, this theory maintains that major traits (e.g., extraversion and neuroticism) originate in individual differences in the sensitivity of these brain systems to their adequately eliciting stimuli.

This theoretical perspective suggests that Archer's theme - that competitive aggression is predominantly a male phenomenon, whereas provoked aggression is equally common in males and females - may be explained by sex differences in functioning of these brain systems. For example, a male who assaults another male with the intention of improving his own mating prospects is displaying appetitively motivated predatory aggression (in this case, by the prospect of sexual intercourse) that is hypothetically mediated by the BAS. In support of this view, there is now mounting evidence that anger/aggression is, at least in part, elicited by BAS activity (Carver 2004; Carver \& Harmon-Jones 2009; Corr 2002). The defensive-versus-predatory and negative-versus-positive distinctions receive additional support from findings that rats seek out opportunities to perpetrate predatory aggression - as if it were appetitive - yet they try to avoid perpetrating defensive aggression - as if it were unpleasant (Panksepp 1971).

If, over time, predatory aggression boosts male fecundity, then this trait would spread throughout the male population by standard evolutionary means. As explained by Archer, the mechanics of mammalian reproduction mean that women face little or no selection pressure to harm or kill female rivals, and so a trait for predatory aggression should not be expected to proliferate in the female population. Given that individual variations are observed in the functioning of the FFFS, BAS, and BIS, personality differences, both within and between the sexes, should be expected in defensive and predatory aggression. For example, a personality dimension, such as impulsiveunsocialised-sensation seeking (often labelled "psychoticism"; Eysenck \& Eysenck 1991) would be a promising candidate for a measure of predatory tendencies. This idea is supported by males typically scoring higher on these traits (e.g., Diaz \& Pickering 1993).

With regard to defensive aggression, an evolutionary view, such as that advanced by Archer, would predict there should be little significant difference between the sexes, as both sexes face selection pressure to defend themselves against aggressive conspecifics and other species. This idea is supported by evidence that females are as likely as males to be aggressive when provoked (Bettencourt \& Kernahan 1997), which is further supported by study of human defence reactions using a threat scenario questionnaire approach (Blanchard et al. 2001). This questionnaire requires participants to select their preferred defensive response to each of 12 threat scenarios from a list of 10 defensive options (e.g., fight, run away). Typically, preferred defensive reactions to scenarios describing especially close or inescapable threats consist of explosive behaviour and aggression (e.g., yell, scream, attack, struggle), and this is equally likely for men and women (e.g., Perkins \& Corr 2006).

If the distinction between defensive and predatory aggression is valid, then a dissociation should exist in human preferences for exhibiting aggression, with both sexes seeking to avoid situations that require defensive aggression, but males (especially those who score high on traits such as psychoticism) preferentially seeking opportunities to perpetrate predatory aggression. This hypothesis has yet to be tested in humans, but it is supported anecdotally by the predominantly male enjoyment of, and 
participation in, rough or violent sports such as boxing, rugby, and American football. It would be valuable to know the importance Archer attaches to the defensive-versus-predatory distinction, and the role played by personality factors in aggressive behaviours; we would be interested in learning about the possible evolutionary pressures on the variation of aggression seen within the sexes.

\section{Two more things for consideration: Sexual orientation and conduct disorder}

\section{doi:10.1017/S0140525X09990252}

\section{Thomas Edmund Dickins ${ }^{a}$ and Mark James Timothy Sergeant ${ }^{\mathrm{b}}$}

a School of Psychology, University of East London, London E15 4LZ, United Kingdom, and Centre for Philosophy of Natural and Social Science, London School of Economics, London WC2A 2AE, United Kingdom; ' ${ }^{\text {S }}$ chool of Social Sciences, Nottingham Trent University, Nottingham NG1 4BU, United Kingdom.

dickins@uel.ac.uk; dickins@Ise.ac.uk mark.sergeant@ntu.ac.uk http://www.uel.ac.uk/psychology/staff/tomdickins.htm http://www.Ise.ac.uk/collections/CPNSS/people/centre_research_ associatesinResidence.htm

http://www.ntu.ac.uk/research/school_research/social/staff/ 54063gp.html

Abstract: We add to Archer's review with mention of sexual orientation differences in aggression and empathy, which suggest a biological basis for the mediating role of empathy. We also note that Archer's view of sex differences will illuminate discussion of conduct disorder, which can only be of help to researchers in this field.

As Archer has made clear, intrasexual competition among males is a consequence of operational sex ratios (OSR). Male gametes are more numerous and cheaper to produce than those of females, and in many species paternal care is relatively low. This results in a situation where there are more reproductively available males than females, and females are thus a scarce resource to be striven for; and consequently, males enter into competition with one another. Those males with heritable traits that enable competitive success reproduce more and those traits go to fixation. The literature abounds with examples of male phenotypes designed for aggressive display and combat, and these dimorphisms are the consequence of this intrasexual selection. Although Archer is asking whether sexual selection accounts for sex differences in aggression, he is really adding to the evidence for sexual selection in his detailed overview of this literature. We want to add two more considerations to the discussion.

One line of enquiry that Archer does not pursue is that of the influence of sexual orientation on aggression. Organizational hormones, important in the process of sexual differentiation, are strongly implicated in the aetiology of male homosexuality. Specifically, the regime of organizational hormones appears to be different in homosexual compared with heterosexual males. These hormones are thought to alter a number of behaviours, somatic features, and cognitive dispositions, including sexual preference (see Wilson \& Rahman 2005). A number of studies have now also drawn a tentative link between the actions of these organizational hormones and the levels of physical aggression displayed by individuals (Bailey \& Hurd 2005; Berenbaum \& Resnick 1997; Fink et al. 2007; Pasterski et al. 2007).

Sergeant et al. (2006) and Dickins and Sergeant (2008) have recently tested specific hypotheses about homosexual male aggression at an individual level and the coalitional psychology underpinning group-level aggression. Both papers report that homosexual males display significantly lower levels of physical aggression than heterosexual males. No differences were recorded in either study for sexual orientation-related differences in verbal aggression, anger, hostility, or several forms of indirect or relational aggression. These findings are in accord with the sex differences described by Archer. Furthermore, both papers documented significantly higher levels of empathy among homosexual males compared to heterosexual males. Empathy is identified by Archer as possible mediator of aggression, associated with the "biosocial" approach (Bettencourt \& Miller 1996; Eagly \& Steffen 1986), and as being reduced among women through the administration of exogenous testosterone (Hermans et al. 2006b). Interestingly, the levels of empathy displayed by individuals have been tentatively linked to organizational hormone exposure (Knickmeyer et al. 2005). Thus, there appears to be a relationship not only between organizational hormones, sexual orientation, and the process of sexual differentiation, but also between levels of physical aggression and empathy.

Although Archer clearly talks about less-than-desirable traits in his paper, he does not mention disorders associated with aggression. For the most part this is unsurprising, for dysfunction is not the focus of his argument. However, conduct disorder might be an exception to this. The American Psychiatric Association describes conduct disorder as "a repetitive and persistent pattern of behaviour in which the basic rights of others or major age-appropriate societal norms or rules are violated" (DSM-IV Diagnostic Criteria 312.8, American Psychiatric Association 2000). To be diagnosed with conduct disorder a person must exhibit three or more behaviours from a list of 15 during the course of one year, with at least one in the past six months. Seven of these behaviours are aggressive and include intimidation, physical fights, and sexual coercion.

Males are two-and-a-half times more likely to have conduct disorder than females. Meltzer et al. (2000), in a survey of the mental health of children and adolescents (from 5 to 15 years old) in Great Britain, reported that conduct disorder was overrepresented in low socioeconomic status boys between 11 and 15 years of age. It is not unreasonable to assume that boys of low socioeconomic status are under-resourced and face more risks than wealthier boys. Given that the former are entering sexual maturity from some point after age 11 years, and their endocrine profile is changing accordingly, the full impact of their local OSR will begin to be felt. As Archer has noted, males from such backgrounds are more likely to be heavy future-discounters and more prone to aggressive conflict, irrespective of a conduct disorder diagnosis (Wilson \& Daly 1997). Finally, it is worth noting that girls with conduct disorder have higher levels of free testosterone (Pajer et al. 2006) and are more likely to have precocious menarche (Burt et al. 2006). Early menarche is also associated with high-risk environments, lower socioeconomic status and early fertility (Belsky et al. 1991; Chisholm 1999; Dickins 2006). As Clutton-Brock (2007) notes, in a recent discussion of advances in sexual selection theory, females of some species do compete aggressively for breeding opportunities, and they can exhibit more masculine anatomical, physiological, and behavioural profiles. This kind of female competition can emerge, even when the OSR is as discussed by Archer.

Taking sexual selection seriously, as Archer does, thus provides us with a possible research programme with regard to conduct disorder. It would be of great value to collect data on the local ecologies in which conduct disorder arises, tracking resources and OSR as well as fertility profiles. The relationship between early fertility, or teenage pregnancy, and male aggression is well known (Wilson \& Daly 1997), but sexual selection theory should throw new light on the facultative psychology underpinning these patterns. 


\section{Sexual selection does not provide an adequate theory of sex differences in aggression}

\section{doi:10.1017/S0140525X09990264}

Alice H. Eagly ${ }^{\mathrm{a}}$ and Wendy Wood ${ }^{\mathrm{b}}$
${ }^{\mathrm{a} D e p a r t m e n t ~ o f ~ P s y c h o l o g y, ~ N o r t h w e s t e r n ~ U n i v e r s i t y, ~ E v a n s t o n, ~ I L ~ 60208-~}$
4609; ${ }^{\mathrm{b}}$ Department of Psychology and Neuroscience, Duke University,
Durham, NC 27708.
eagly@ northwestern.edu
wwood@duke.edu
http://www.wcas.northwestern.edu/psych/people/faculty/faculty_
individual_pages/eagly.htm
http://www.duke.edu/ wwood

Abstract: Our social role/biosocial theory provides a more adequate account of aggression sex differences than does Archer's sexual selection theory. In our theory, these sex differences arise flexibly from sociocultural and ecological forces in interaction with humans' biology, as defined by female and male physical attributes and reproductive activities. Our comments elaborate our theory's explanations for the varied phenomena that Archer presents.

John Archer's stimulating article compares our social role/biosocial theory (see Wood \& Eagly 2002) with his own version of sexual selection theory as accounts for sex differences in aggressive behavior. We are delighted to see the increasing importance he gives in his own theory to men's and women's social roles and to understanding the dynamic biosocial interactions that produce sex differences in behavior.

A central difference, however, remains between our perspective and his - we allocate greater causal power to social structural determinants of male and female aggression and less to sex-differentiated aggressive dispositions presumed to be built into human psychology through sexual selection. Additionally, Archer fails to acknowledge empirical evidence that challenges the sexual selection aspects of his theory, and he misses key points in presenting our work.

Human evolution yields behavioral flexibility. Any evolutionary analysis of human sex differences has to account for humans behavioral flexibility. This flexibility in response to local circumstances is a characteristic feature of the human species. It reflects their evolution in diverse environments with changeable conditions that impinged in differing ways on survival and reproductive outcomes (Wood \& Eagly 2002; in press). For example, in human history, particularly in the late Pleistocene era, climate appears to have been highly changeable. Also, humans and their ancestors engaged in extensive niche construction, meaning that their activities produced changes in the environments in which they lived. Accommodating to such changes required behavioral flexibility, enabled by an evolved capacity for innovating and sharing of information through social learning, yielding a cumulation of culture (Richerson \& Boyd 2005). Humans' flexibility is evident in their various novel solutions to the problems of reproduction and survival, including tolerance for a wide range of foods, ecologies, and living arrangements.

This flexibility in behavior is at the heart of our evolutionary analysis. Sex differences in behavior arise flexibly from a biosocial interaction, in particular from sociocultural and ecological forces in interaction with humans' biology as defined by female and male physical attributes and reproductive activities (Wood \& Eagly 2002). Women bear and nurse children, and men possess greater size, speed, and upper-body strength. These attributes serve as constraints on behavior such that certain activities are more efficiently accomplished in certain societies by one sex than by the other. Consequently, the sexes participate in a division of labor. Some behaviors tend to be performed by one sex across most societies and time periods, but even these behaviors can be influenced by particular societal circumstances.
In vivid illustration of the flexibility of sex differences in human aggression, women sometimes, although rarely, engage in organized combat in world societies. A prominent example is the "Amazon Corps" of the Dahomey Kingdom of West Africa in the eighteenth and nineteenth centuries. Because endemic warfare had reduced the supply of male warriors, survival of the kingdom required military service by women. These women were trained for combat and fought with distinction in all-female units (e.g., Alpern 1998). A prominent twentieth century example is: the women soldiers of Eritrea. These women fought successfully alongside men in mixed units in the long-term and eventually victorious struggle of the Eritrean People's Liberation Front to win Eritrea's independence from Ethiopia (Bernal 2000). Eritrean women's military service was apparently ideologically driven by the revolutionary movement's modernist rejection of traditional gender relations. These societies overcame the constraints of female reproduction in different ways - in Dahomey by forbidding women warriors to have sex with men, and in Eritrea by providing community childcare. The potential military disadvantages of lesser female physical prowess were surmounted through training and the provision of weapons. In both societies, the female warriors' psychology proved adequate for full participation in highly aggressive combat activity. Also indicative of flexibility in aggressive behavior is the evidence that women in a small percentage of world societies regularly hunted large game (see Wood \& Eagly 2002).

Division of labor produces sex differences in human behavior. Archer has left out the key link in our theory between the division of labor and gender roles, which explains how these roles and associated behaviors change over time and circumstance. Consistent with the social psychological principle of correspondent inference (Gilbert 1998), people infer the traits of men and women from observations of their behavior. To the extent that people observe men and women engaging in different types of behaviors, they regard them as psychologically different. These observations underlie gender roles, which form a shared knowledge structure specifying what men and women usually do and what they should do in a society.

These gender roles, as well as specific social roles such as daughter, boss, and friend, influence behavior through a trio of interacting proximal causes of sex differences and similarities: Roles influence behavior through chemical signals of hormonal changes in interaction with individuals' personal gender identity and others' stereotypic expectations. Our position that the activating influence of hormones is mainly to facilitate role behavior reflects Archer's excellent meta-analysis (Archer 2006b). Specifically, testosterone rose in men anticipating and playing sports and highly competitive games, especially among the contest winners, but did not rise in the absence of social roles or provocations calling for aggressive, dominant behavior. Although women have substantially less testosterone than do men, female athletes also recruit testosterone before a competition (see Wood \& Eagly, in press). But Archer's (2006b) meta-analysis did not show the reverse causal relation: Studies that experimentally injected men with testosterone or related synthetic androgens found no systematic rise in anger, aggression, or hostility resulting.

Along with hormonal changes, internalized gender roles produce gender identities that act as trait-like determinants of aggressive behaviors. Others' expectations also foster behavior consistent with gender roles. Unfortunately, Archer misinterpreted one of the best-designed experiments demonstrating that aggression is influenced by others' expectations (Lightdale \& Prentice 1994). This study did not confound the directness of aggression with its normative manipulation, as he claimed, and thus demonstrated how sex differences in aggression depend on the salience of social norms. Nonetheless, Archer is correct in recognizing our theory's trio of psychological mechanisms (hormonal activation, gender identity, others' expectations) 
that can yield differences between men and women in aggressive behaviors as well as differences between individuals within each sex.

Sexual selection provides an inadequate account of human bodily dimorphism. Archer maintains that men's size and strength and other physical attributes were sculpted by sexual selection pressures in which ancestral males who were larger and stronger had better fitness outcomes because they were able to compete with other males for access to many mates. Sexual selection pressures presumably also organized human psychology, making men more aggressive than women.

Comparative research with primates, however, suggests that bodily dimorphism requires a more complex explanation. Despite the bodily metrics that Archer presents, when evaluated in relation to other anthropoid primate species, humans have relatively low male-female dimorphism in both body weight and canines, and presumably in other bodily attributes as well (Plavcan \& van Schaik 1997a, p. 351). Even though across all primate species, greater bodily dimorphism was associated with polygynous mating and male-male competition, dimorphism at the low levels existing in humans "can be found among species with a wide variety of mating systems and competition levels" (Plavcan 2000, p. 338). In addition, compared with most other primate species, humans have a low operational sex ratio (e.g., Wrangham et al. 1999), which also is compatible with low male-male competition. We are puzzled by Archer's failure to acknowledge that, when compared with other primates, the relatively small amount of human size dimorphism does not imply sexual selection through malemale competition.

Also undermining Archer's one-dimensional sexual selection account is evidence that bodily dimorphism was likely influenced by selection on females as well as males. Selection pressures on females are plausible, given that the decreasing size dimorphism as hominids evolved from the earlier Australopithecus to Homo was due to an increase in the size of females relative to males (as Archer notes). For this and other reasons, experts have abandoned Archer's one-sided sexual selection argument in favor of a richer set of possibilities. These newer ideas include not only selection pressures on females but also more varied principles for understanding the cooperative and competitive relations between males and the niche construction of males and females in varied environments (e.g., Plavcan 2000; Plavcan \& van Schaik 1997a; 2005).

Now consider the real predictions of the social role/biosocial theory. It is troubling that Archer claims that we believe that sex differences in aggression are "modest" (see his Table 1). Our analysis yields no a priori hypotheses about the size of sex differences but instead anticipates that they pattern in a society according to the trio of proximal causes noted above. In addition, as we explain in the next paragraphs, Archer has freely invented (and then disproved) a social role prediction about developmental changes in aggression sex differences and failed to recognize an obvious social role alternative prediction for greater male than female variability in aggressiveness. He also oversimplifies the social role predictions for cross-cultural comparisons of sex differences.

Archer maintains that our position on developmental changes should be that, "Sex difference should start small and increase with age through childhood, coincident with the cumulative influence of socialization" (see Table 1 of the target article). This naïve prediction assumes that socialization pressures cumulate in some simple way across development, and furthermore, that they overwhelmingly encourage boys' aggressiveness - that they are composed of cheering sections of mothers, fathers, teachers, siblings, and peers, all urging aggressive behavior. On the contrary, boys encounter both discouragement of aggression (e.g., in the classroom) and encouragement (e.g., in sports, selfdefense). Prohibitions against physical aggression increase as boys mature, with violence disallowed in the overwhelming majority of employment settings and disparaged in close relationships with friends, family, and romantic partners. Given these complexities, boys learn to express aggression contextually and in patterned ways. We have not made the claim that Archer suggests but instead have remained silent on age trends in aggression because prediction demands close study of the pressures that foster and discourage aggression as boys and girls mature.

With respect to within-sex variability in aggressive behavior, our theory offers an obvious alternative explanation to sexual selection theory. Societies have often provided more opportunities for boys than girls to learn aggression (e.g., in gangs and contact sports). Because some boys and young men avail themselves of these opportunities and others do not, their aggressiveness should be more variable than that of women and girls. Men's greater power and resources in society also bring some men protection from retaliation, and other, subordinate men greater vulnerability to retaliation. Women's more restricted opportunities are consistent with their lesser variability in these behaviors.

Finally, we note that many cross-national comparisons of sex differences, although seemingly an attractive way of testing social role and evolutionary hypotheses, are plagued by ambiguity. Our theory generally predicts a lessening of sex differences with greater gender equality - and this is what Archer (2000a) found with greater male physical aggression to partners in countries marked by lesser gender equality. Yet, gender equality is not the only feature of men's and women's roles that influence sex differences across cultures. Other aspects of roles influence subjective ratings of personality attributes and abilities. In particular, the extent of segregation of men and women can influence the comparison standard that they use to evaluate themselves and others. In traditional cultures in which occupational and other roles tend to be segregated by sex, men and women would judge their own and others' psychological attributes through comparisons with salient others, who would mainly be of the same sex. Thus, a man might rate himself as only moderately aggressive because he is comparing himself with other men, who are generally somewhat aggressive in his society. In contrast, in societies with less segregated roles, a man might compare himself with individuals of both sexes and conclude that he is relatively aggressive. The result of this shifting comparison standard is that sex differences can appear to be smaller in less egalitarian, more hierarchical societies, in which individuals compare themselves to their own sex (Guimond et al. 2007). Therefore, cross-national comparative data based on subjective trait ratings (e.g., Schmitt et al. 2008) cannot be taken at face value. Overcoming such confounds requires common-rule (or more objective) measures that disallow standard shifts (Biernat 2003). The reports of behavioral frequencies in the research on intimate partner aggression are an instance of common-rule measures, thus allowing for confirmation of our social role prediction in Archer's (2000a) metaanalysis.

In conclusion, the debate between sexual selection theories of the origins of human sex differences and our social role/ biosocial theory will no doubt continue as proponents of each theory hone their views in response to newly emerging empirical evidence. We are encouraged by Archer's extension of standard evolutionary psychology models to include social roles, but we think an adequate theory of sex differences in aggression would take more seriously the flexibility in behavior that follows from a social role analysis.

\section{ACKNOWLEDGMENT}

The authors thank Paul Eastwick for his comments on a draft of this commentary. 


\section{Sex, aggression, and life history strategy}

doi:10.1017/S0140525X09990422

\section{Aurelio José Figueredo, ${ }^{a}$ Paul Robert Gladden, ${ }^{a}$ and Barbara Hagenah Brumbach ${ }^{\mathrm{b}}$}

${ }^{a}$ Department of Psychology, University of Arizona, Tucson, AZ 85721-0068; ${ }^{\mathrm{b}}$ Department of Psychology, Northern Arizona University, P. O. Box 15106, Flagstaff, $A Z$ 86011-5036.

ajf@u.arizona.edu www.u.arizona.edu/ ajf

pgladden@email.arizona.edu

barbara.brumbach@nau.edu

Abstract: We agree that sexual selection is a more comprehensive explanation for sex differences in direct aggression than social role theory, which is an unparsimonious and vestigial remnant of human exceptionalism. Nevertheless, Archer misses several opportunities to put the theoretical predictions made by himself and by others into direct competition in a way that would further the interests of strong inference.

Archer argues that sexual selection is a more comprehensive explanation for sex differences in direct aggression than social role theory. We concur completely with this assessment. Indeed, we find it hard to believe that social role theory, even the "biosocial" version, retains any scientific credibility at all in the twenty-first century. To us, social role theory is a vestigial remnant of human exceptionalism. Given the overwhelming preponderance of comparative evidence for sexually selected sex differences in intraspecific aggression across such a broad diversity of species, it does great violence to the principle of parsimony to invent a special explanation for exactly the same phenomenon in our own species. Surely, such special pleading cannot be considered sound scientific theorizing.

Although we agree that sexual selection theory is essential for explaining sex differences in aggression, Archer seems indecisive regarding the relationship between sexual selection theory and the influence of social roles. Archer suggests that evolved dispositions typically do not rely on socialization practices that could vary from culture to culture (sect. 2.1.2). However, he acknowledges that several ecological and social conditions (e.g., scarcity of resources, operational sex ratio, and culture of honor) are predicted to modify the sex difference magnitude according to the sexual selection view. Here, Archer's argument would have been aided by emphasizing that evolved dispositions and the influence of social context are not in competition with one another. Further, Archer's analysis of intrasexual aggression would have been strengthened by specifying why the sexual selection theory of sex differences in aggression is consistent with epigenetic sensitivity to particular environmental conditions, rather than emphasizing insensitivity to those specified by social role theory. This emphasis becomes more difficult to understand in the light of the discussion of intersexual aggression in which sexual selection theory and social role theory are treated as complementary explanations. This leaves the reader confused about why parts of the social role explanation, or at least similar social conditions, could not be integrated with the sexual selection explanation.

The analysis would have been further strengthened by specifying the differences between the two theories at both the ultimate and proximate levels of explanation. Advocates on both sides of this debate should attempt to explicitly specify what their theories imply about both levels, because there is a tendency to contrast the ultimate level of sexual selection theory with the proximate level of social role theory. Although Archer does present proximate level mediators specified by each theory, it might have been helpful to examine what social role theory implicitly implies about the ultimate level. Each theory implies some differences at both levels, and an effort should be made to translate the two models into the language of each level to allow more thorough examination.

Archer acknowledges there is evidence for an association between "internalization of gender roles" and measures of aggression (sect. 2.9). However, in our own lab, we have found evidence that individual differences in both mating effort and life history (LH) strategy are associated with both sexual aggression (Gladden et al. 2008) and negative androcentrism (bias against women; Figueredo et al., in preparation), suggesting that sexual selected LH strategies may account for the association between so-called gender roles and aggression. Future work should attempt to put the sexual selection and social role models in direct competition. The use of path-analytic modeling may suggest which model fits the data best.

Although Archer's primary point was to discuss sex differences in physical aggression, we would suggest that expanding a discussion of sex differences in indirect aggression could have strengthened his argument that sexual selection plays an important role in determining both between and within sex differences in aggression. The difference between males and females in indirect aggression may not be as substantial as that of physical aggression; however, the evidence he presents on effect size clearly indicates that indirect aggression is employed more by females than by males (sect. 2.8). Why is this? Archer's use of sexual selection theory for explaining sex differences in physical aggression could be applied equally well for examining sex differences in indirect aggression. The following are just a few examples of how this logic could be applied.

Archer suggests that, "Overall, the most likely prediction from a sexual selection perspective is an early emergence of sex differences in aggression combined with a peak in risky competition during young adulthood" (sect. 2.1.2, para. 3). One could make the same prediction focusing on indirect rather than physical aggression. More specifically, one could predict the following: (1) females will begin engaging in indirect aggression at an early age (e.g., grade-school girls negotiating social networks at recess), and (2) female indirect aggression will increase into adolescence and young adulthood (e.g., junior-high girls gossiping about rivals at lunch).

Archer also addresses context and within-sex competition, "Sexual selection predicts variability in response to conditions that affect the extent of inter-male or inter-female competition, notably resources that are important for reproduction, such as access to mates, and the status and resources important in this process" (sect. 2.8, para. 1). The same logic could be used to further predict differences in indirect aggression among females. For example, one could predict that: (1) there should be less indirect aggression from females who have formed satisfactory and relatively secure long-term pair bonds or secured adequate resources; (2) there should be more indirect aggression from females who pursue short-term mating strategies (fast LH strategy) and less indirect aggression from females who pursue long-term mating strategies (slow LH strategy), especially once a secure pair bond is established (i.e., the less secure the bond, the more incidents of indirect aggression); and (3) female indirect aggression should focus on issues of contested resources, relative fertility, and paternity certainty.

In short, Archer misses several opportunities for putting the theoretical predictions made by both himself and others into direct competition in a way that would further the interests of strong inference (Platt 1964). Hence, although essentially correct, in our view his critique did not go far enough in demolishing the antiquated and obsolete alternative hypotheses reviewed. 


\section{An $I^{3}$ Theory analysis of human sex differences in aggression}

\section{doi:10.1017/S0140525X09990410}

\section{Eli J. Finkel and Erica B. Slotter}

Department of Psychology, Northwestern University, Evanston, IL 60208. finkel@northwestern.edu ericaslotter2011@u.northwestern.edu http://faculty.wcas.northwestern.edu/eli-finkel/

Abstract: According to $\mathrm{I}^{3}$ Theory, individuals enact aggressive behaviors when (a) instigating triggers are severe, (b) impelling forces are strong, and/ or (c) inhibiting forces are weak. Archer's analysis of human sex differences in aggression could be bolstered by a careful analysis of male-female discrepancies in reactivity (or exposure) to instigating triggers, proneness toward impelling forces, and/or proneness toward inhibiting forces.

Any comprehensive theory of human sex differences in aggression must accomplish the following three tasks (among others). First, it must establish the presence and magnitude of these sex differences. Second, it must discern which specific mediators (e.g., risk-taking, fear of danger) account for these differences. And third, it must identify the specific mechanism by which these mediators translate into behavior (e.g., by strengthening aggressive urges, by weakening the restraint of such urges).

Archer's impressive review, which does not purport to be a comprehensive theory of sex differences in aggression, focuses on the first and the second of these tasks. Regarding the first, relative to women, men are considerably more physical aggressive (in Archer's Table 2 , average $d=.58$, range $=.33-.91$ ) and somewhat more verbally aggressive (average $d=.29$, range $=.09$ $.55)$, although women are slightly more indirectly aggressive (average $d=-.16$, range $=-.45-.05$ ). Regarding the second, sex differences in aggression appear to be driven in large part by male-female discrepancies in factors such as risk-taking (men are higher) and fear of physical danger (women are higher).

We believe Archer's analysis could be bolstered by a careful analysis of the third task. According to $I^{3}$ Theory (pronounced "ICubed Theory"), scholars can determine whether an individual will engage in aggressive behavior in a given situation by discerning the strength of the relevant instigating triggers, impelling forces, and inhibiting forces (Finkel 2007; 2008; Slotter \& Finkel, in press). Instigating triggers refer to discrete, situational events or circumstances that induce rudimentary action tendencies toward physical aggression (e.g., provocation, goal obstruction, opportunities for personal gain). Impelling forces refer to the collective power of factors that increase the strength of individuals' tendencies to experience aggressive urges in response to an instigating trigger (e.g., high dispositional anger, elevated testosterone, previous exposure to violent media). Individuals tend to experience more powerful aggressive urges when impelling forces are strong than when they are weak, especially when instigating triggers are severe. Inhibiting forces refer to the collective power of factors that increase the strength of individuals' tendencies to override aggressive urges rather than acting upon them (e.g., high dispositional self-control, strong relationship commitment, sobriety). Inhibiting forces function as a threshold: Individuals will enact aggressive behavior only when aggressive urges are stronger than inhibiting forces.

Archer suggests that the tendency for males to be more aggressive than females is likely to be mediated by greater male risktaking (for reproductive advantage) and greater female fear of physical danger. From the perspective of $\mathrm{I}^{3}$ Theory, the former could plausibly function as an impelling factor causing men to experience stronger aggressive urges than women because access to mates is so enticing, whereas the latter could plausibly function as an inhibiting factor causing women to experience stronger restraint of aggressive urges than men because of the elevated costs of acting upon these urges. If so, men are more aggressive than women because men experience both stronger impelling tendencies toward aggressive urges and weaker inhibiting tendencies to restrain these urges than women do. Establishing definitively whether risk-taking is an impelling factor and whether fear of physical danger is an inhibiting factor, however, is an important direction for additional empirical research.

Regarding instigating triggers, Archer argues that the sexual selection analysis implies that the mechanism underlying sex differences in aggression "is unlikely to reside in a general sex difference in response to frustration or ease of arousal to anger" (sect. 2.1.2, para. $5)$. It is not immediately obvious to us why sexual selection would have built men to be (a) more (directly) aggressive than women while simultaneously (b) no more anger-prone in general or reactive to instigating triggers in particular. Many scholars argue that anger is an emotion that evolved in large part for its aggression-related consequences (e.g., Fischer \& Roseman 2007; Frijda et al. 1989), so the disconnect between anger and aggression in Archer's model (implying that the link between anger and aggression differs for men and women) requires further elaboration.

The $\mathrm{I}^{3}$ Theory emphasis on instigating triggers, impelling forces, and inhibiting forces is also relevant to cases where male/female levels of aggression are comparable. One such instance is physical aggression in heterosexual romantic relationships. According to Archer's review, "there are no appreciable sex differences in physical aggression to opposite-sex partners, and therefore there is no need to look for ultimate explanations or for mediators" (sect. 4.4, para. 2). From the perspective of $\mathrm{I}^{3}$ Theory, this latter conclusion may be premature. It seems plausible that there could be ultimate explanations (and also proximal explanations) for sex differences in reactivity (or exposure) to instigating triggers, in the experience of impelling forces, and/or in the experience of inhibiting forces that trend in opposite directions and consequently neutralize one another. For example, perhaps sexual selection has caused men to experience stronger impelling tendencies to aggress physically toward an opposite-sex romantic partner (consistent with men's tendency to be more physically aggressive in general), but this effect is neutralized by stronger inhibiting tendencies for men to restrain these urges (particularly in cultural contexts where boys and men are socialized that it is inappropriate to be physically aggressive toward girls and women). Future research could productively explore whether sex-differentiated tendencies across the $\mathrm{I}^{3}$ Theory components could account for the lack of appreciable sex differences in the frequency of physical aggression toward opposite-sex partners.

In sum, although Archer's analysis of human sex differences in aggression is timely and valuable, it could benefit from greater elaboration of the psychological mechanisms driving these differences. Identifying mediators like risk-taking and fear of physical danger is a step in the right direction, but doing so does not establish whether the sex differences result from male-female discrepancies in reactivity (or exposure) to instigating triggers, proneness toward impelling forces, and/or proneness toward inhibiting forces.

\section{ACKNOWLEDGMENTS}

The preparation of this commentary was supported in part by the National Science Foundation (Grant No. 719780). Any opinions, findings, and conclusions or recommendations expressed in this material are those of the authors and do not necessarily reflect the views of the National Science Foundation. We thank Paul Eastwick for his constructive feedback on an earlier draft.

\section{Biophobia breeds unparsimonious exceptionalism}

\section{doi:10.1017/S0140525X0999029X}

\section{Steven J. C. Gaulin}

Department of Anthropology and Center for Evolutionary Psychology, University of California, Santa Barbara, Santa Barbara, CA 93106-3210. gaulin@anth.ucsb.edu

Abstract: With respect to aggressiveness it is not enough to say that humans are "like other mammals." We resemble only those species 
where males have higher maximum reproductive rates than females. In such species males evolve a set of hormonally mediated competitive traits via sexual selection. Because humans match the predictions of this general evolutionary model, attempts to (re)explain men's aggressiveness in sociological terms are superfluous and misleading.

Archer compares sexual selection (Darwin 1871/1901) and social role theory (Eagly 1987) as explanations for human sex differences in aggressiveness. Archer's empirical claims are grounded, but his case is weakened because he fails to emphasize two related metamethodological points: the power of the comparative approach and the parsimony it provides. Greater male aggressiveness is neither uniquely human nor universal among animals. It exhibits a particular cross-species distribution, being present in some species and absent in others. That distribution strongly constrains its functional explanation. Unfortunately, social role theory neglects this powerful source of insight. It examines sex differences in aggression in a zoological vacuum, naively treating the human case as unique. As a result of its narrow focus, social role theory fails to shed new light on sex differences in human aggression. Like many models in the social sciences, it is a case of special pleading where none is required.

Darwin (1871/1901) defined sexual selection as an evolutionary process that regularly produces differences between the females and males of a species, and he enumerated the reproductive differentials that drive it. Subsequently, twentieth-century biologists (Andersson 1994; Clutton-Brock \& Vincent 1991; Trivers 1972) explained the forces that give sexual selection its polarity. It is this polarity - which sex is more aggressive - that both sexual selection and social role theory seek to explain.

Scientific theories explain relevant variance. Thus, social role theory would have some traction if there were cultures where women are more aggressive than men. For better or worse, no such cultures exist (Brown 1991; Daly \& Wilson 1988). So, where is the theory-testing variance? To find it one must escape anthropocentric Durkheimian biophobia and look across species.

Sexual selection theory provides a general explanation of sex differences that applies to all sexual species. It predicts the distribution of sex differences in aggression: which species will evolve greater male aggressiveness, which species will exhibit no such sex differences, and which will show greater female aggressiveness. According to sexual selection theory, aggressiveness is not a function of sex per se, but of sex differences in maximum reproductive rate (Clutton-Brock \& Vincent 1991), arising out of sex differences in parental investment (Trivers 1972). To illustrate, because only female mammals gestate and lactate, a male could have many more offspring than a female. Every reproductive venture requires one male and one female; thus the slower sex is in short supply and worth competing for. (In this example reproductive physiology determines reproductive rates but aspects of the mating system may also be important.)

Sexual selection theory's accuracy in predicting the distribution of sex differences across species makes it logically prior to ad hoc explanations of sex differences in any particular species, unless that species fails to match its predictions. Thus, a baseline question for social role theorists is, do humans constitute an exception to sexual selection theory? Men have, and have had for thousands of generations, higher maximum reproductive rates than women. This implies that men will have found women in short supply and consequently evolved a suite of competitive tactics for acquiring mates, including aggression.

What remains for social role theory to explain? Its proponents might say "development." But, whatever ontogenetic influences social scientists imagine for gender roles, their hypotheses will have to contend with a thick cross-species literature on the developmental effects of androgens. Wherever sexual selection has produced more aggressive males, androgens orchestrate the development of that sex difference. As a functional viewpoint would suggest, the very same hormones also shape the anatomical components of the male-competition complex. Compared to women, men are much stronger and more muscular in the upper body (the region most engaged in physical aggression).

The effect size for these sex differences is approximately 3.0, with $99.9 \%$ of women falling below the male mean; individual differences in muscle mass still reliably predict male mating success in the United States (Lassek \& Gaulin, in press). Thus, sexual selection simultaneously explains both anatomical and behavioral sex differences and their joint hormonal mediation. Social role theory cannot approach this level of explanatory integration.

But the explanation gap is wider still. The male-competition complex has many evolved manifestations. Bimaturism, sexually differentiated mortality rates, and sex differences in navigational ability are well-described human traits; and again, the crossspecies distribution of these traits strongly implicates sexual selection. Delayed sexual maturity of males is limited to species where they have higher reproductive rates than females (Leigh 1992, Leigh \& Shea 1995). Controlled within-genus comparisons suggest that searching for mates drives the evolution of male navigational ability, but again, only where they have higher reproductive rates (Gaulin 1992). Both bimaturism and navigational ability are developmentally linked to the same androgenic hormones that organize aggressive structures and behaviors. Many aspects of the male-competition complex entail costs reflected in higher male mortality rates: In both birds and mammals sex differences in mortality are not universal but proportionate to the intensity of sexual selection (Promislow 1992, Promislow et al. 1992). These costs are not limited to combat-related mortality but include higher male susceptibility to infection that: (a) closely tracks the intensity of sexual selection across species (Moore \& Wilson 2002), (b) manifests prominently in humans (Owens 2002), and (c) is probably related to androgens' immunosuppressant effects (Folstad \& Karter 1992) because castration removes the sex difference in infection rates and hormone replacement reinstates it (Zuk \& McKean 1996). It is not the existence of these traits but their patterned, cross-species association that social role theory must confront.

In sum, humans exhibit a suite of traits - elevated male aggressiveness, greater male muscularity and strength, later male maturation, superior male navigational ability, and higher male mortality, all underpinned by an androgen-based developmental system - that they share only with species where male reproductive rates can significantly exceed those of females. The coherent distribution of these traits strongly suggests they were jointly produced by sexual selection. This patterning, revealed by cross-species comparison, supports the causal primacy of sexual selection. In the absence of a significant misfit with the predictions of sexual selection, any attempts to (re)explain men's greater aggressiveness in purely sociological terms constitute unparsimonious exceptionalism and have little scientific promise.

\section{A history of war: The role of inter-group conflict in sex differences in aggression}

\author{
doi:10.1017/S0140525X09990409
}

\section{Dominic D. P. Johnson ${ }^{\mathrm{a}}$ and Mark van Vugt ${ }^{\mathrm{b}}$ \\ ${ }^{a}$ Department of Politics and International Relations, University of Edinburgh, Edinburgh, EH8 9LD, Scotland, United Kingdom; ' Department of Psychology, University of Kent at Canterbury, Canterbury CT2 7NP, England, \\ United Kingdom. \\ dominic.johnson@ed.ac.uk http://www.dominicdpjohnson.com/ \\ M.van-Vugt@kent.ac.uk \\ http://www.kent.ac.uk/psychology/department/people/van-vugtm/}

Abstract: Human aggression has two important dimensions: withingroup aggression and between-group aggression. Archer offers an 
excellent treatment of the former only. A full explanation of sex differences in aggression will fail without accounting for our history of inter-group aggression, which has deep evolutionary roots and specific psychological adaptations. The causes and consequences of inter-group aggression are dramatically different for males and females.

Human aggression takes two very different forms: (1) intra-group aggression (between individuals); and (2) inter-group aggression (between groups of individuals, such as coalitions, gangs, warriors, armies). Archer argues that observed sex differences in aggression are best explained by sexual selection theory, but this is based on an exclusive focus on intra-group aggression, ignoring the potential explanatory (or confounding) role of inter-group aggression.

We suggest that the inter-group dimension is vital to understanding sex differences in aggression: If inter-group processes explain some of the variance in sex differences in aggression, then Archer may have overestimated the role of sexual selection in accounting for the observed sex differences, and may also have underestimated sex differences in aggression overall (since they may be even higher in inter-group contexts).

Inter-group aggression has arguably been a major force in human evolution. There is evidence that warfare was frequent and severe throughout human history (Gat 2006; Guilaine \& Zammit 2004; Keeley 1996; LeBlanc \& Register 2003) and has deep roots in human evolution (Alexander 1987; Thayer 2004; Wrangham \& Peterson 1996). Warfare has been a significant cause of male deaths $(13-15 \%$ in the archeological and ethnographic record; Bowles 2006), suggesting a strong selection pressure on adaptations for inter-group aggression.

Studies of warfare differ in many respects but are in agreement on one thing: it is almost exclusively a male phenomenon (Potts \& Hayden 2008; Wrangham \& Peterson 1996). Although women commonly aid in war efforts of various kinds, they generally do not participate as warriors. Legends of Amazons and female warriors are so rare (or unsubstantiated) as to serve as exceptions that prove the rule. The introduction of women into combat units in modern militaries has also been problematic (Browne 2007). We should, therefore, expect significant sex differences in adaptations to inter-group aggression.

Inter-group aggression introduces at least two complexities to Archer's analysis. First, as noted above, some variance in sex differences in aggression is likely to derive from inter-group processes, not sexual selection. Second, inter-group aggression can often be a cause of reduced aggression between males of the same group - uniting to fight a common enemy. Indeed, extraordinary cooperation (even self-sacrifice) can emerge in the context of inter-group aggression (McNeill 1995; Rielly 2000). Sex-differentiated aggression in inter-group contexts is as much about intermale cooperation as it is about inter-male aggression.

Empirical evidence supports two key predictions of this "male warrior hypothesis" (van Vugt et al. 2007). First, in situations of inter-group threat, men should display more aggression than women. This is a robust finding in both experimental and realworld studies (Johnson et al. 2006; McDermott \& Cowden 2001; Wrangham \& Wilson 2004). Second, in situations of intergroup threat, men should increase their cooperation with the in-group in order to more effectively defend and aggress against the out-group. This is supported by experiments in which cooperation in collective action games increases in the presence of rival groups, but only among men (van Vugt et al. 2007).

An inter-group perspective raises the question of interactions between sexual selection and inter-group aggression: what is the impact of sexual selection on aggression between members of different groups? Indeed, inter-group aggression may actually be rooted in sexual selection. For example, performance in intergroup warfare may bring status or rewards that increase individual reproductive success (Chagnon 1988). Or, since a primary function of wars in pre-industrial societies is the capture of women (Keeley 1996), warfare may represent competition for reproductive access fought between coalitions rather than between individuals. Finally, inter-group aggression may even be a method of displacing sexual competition from the ingroup to the out-group, serving to minimize within-group conflict (and its associated costs).

An inter-group perspective also raises the question of the role of women in aggression. If women have been beneficiaries and victims of inter-group aggression, we would expect selection pressures on response strategies. For example, there is some evidence that women find military men more sexually attractive, but only if they are observed in battle (Leunissen \& van Vugt, unpublished). Women also show an aversion to out-group males at peak fertility in their menstrual cycle (Navarrete et al. 2009). Women might even support inter-group aggression if they (or their offspring and kin) will benefit from the consequences. Keeley reports that among the Apache, "when the meat supply of a band began to run low, an older woman would complain publicly and suggest that a raid be mounted to obtain a fresh supply" (Keeley 1996, p. 135).

An inter-group perspective is also important for Archer's analysis of intersexual (male on female) aggression. Archer focuses primarily on aggression among partners. However, differences in male and female aggression is likely to be highly dependent on group membership. As noted above, a common objective of pre-industrial warfare is the capture of women, and the occurrence of rape in wartime is widely documented even among modern societies (Naimark 1995; Potts \& Hayden 2008). Therefore, male aggression against women is likely to be significantly underestimated if we look only at data on partners men and women who typically chose to be together in the first place, or at least come from the same in-group.

An inter-group perspective does at least support Archer's rejection of social role theory. Briefly, differences in intergroup behavior between boys and girls also appear at a young age and follow a fairly stable developmental trajectory across contexts (Ellis et al. 2008), suggesting an evolutionary explanation. For example, boys more often play team games involving larger groups and have more transient friendships, whereas girls have more exclusive friendships. Boys are also angrier about rulebreaking behavior in such games.

To summarize, inter-group aggression might seem to have little bearing on Archer's core claims - perhaps just representing a different research question. However, we suggest that the omission of an inter-group dimension is significant, because: (1) it underestimates overall sex differences in aggression; and (2) observed sex differences in aggression may derive from some third factor other than sexual selection - in particular intergroup psychology. Thus, even if the evidence that Archer examines is correct, we cannot tell whether it derives from an evolutionary history of sexual selection or from an evolutionary history of inter-group aggression (or some combination thereof). Sex differences in aggression between groups remains an important research area for the future with implications for understanding, predicting, and intervening in human aggression within both domestic and international contexts.

\section{Suspicions of female infidelity predict men's partner-directed violence}

doi:10.1017/S0140525X09990392

\author{
Farnaz Kaighobadi and Todd K. Shackelford \\ Department of Psychology, Florida Atlantic University, Davie, FL 33314. \\ fkaighob@fau.edu \\ tshackel@fau.edu \\ http://www.toddkshackelford.com/
}

Abstract: Archer's argument regarding sex differences in partner violence rests on a general account of between-sex differences in 
reproductive strategies and in social roles. However, men's partnerdirected violence often is predicted by perceived risk of female infidelity. We hypothesize that men's partner-directed violence is produced by psychological mechanisms evolved to solve the adaptive problem of paternity uncertainty.

Archer presents a comprehensive account of between-sex aggression from an evolutionary perspective built on sexual selection theory. We appreciate Archer's argument that sex differences in reproductive strategy are responsible for sexual conflict and for between-sex aggression. Sexual selection explains sex differences in aggression, in general. We contend, however, that there is a particular area of work that deserves more attention in research on violence in intimate relationships. There is a large body of research investigating men's partner-directed violence as an evolved solution to the adaptive problems of female infidelity and paternity uncertainty.

Over the course of human evolutionary history, men have faced the adaptive problem of female sexual infidelity and subsequent cuckoldry - or the unwitting investment in genetically unrelated offspring. The reproductive costs of cuckoldry, including loss of time, energy, resources, and alternative mating opportunities are potentially so great that men are hypothesized to have evolved psychological mechanisms that function to motivate anticuckoldry tactics. The problem of paternity uncertainty is hypothesized to have selected for the emotion of male sexual jealousy, which in turn motivates men's anti-cuckoldry tactics such as nonviolent and violent mate retention behaviors. Considerable evidence indicates that men's perceptions of their female partner's infidelity predict men's partner-directed insults, sexual coercion, and partner-directed violence.

Male sexual jealousy is one of the most frequently cited causes of men's partner-directed violence, both physical and sexual (e.g., Buss 2000; Daly \& Wilson 1988; Daly et al. 1982; Dobash \& Dobash 1979; Dutton 1998; Frieze 1983; Gage \& Hutchinson 2006; Russell 1982; Walker 1979). The frequency with which men perform nonviolent mate retention behaviors predicts the frequency with which they inflict physical violence against their partners, arguably because both classes of behavior are outputs of sexual jealousy (Shackelford et al. 2005a). Men who directly accuse their partners of sexual infidelity also are more likely to inflict partner-directed violence (Kaighobadi et al. 2008).

Sexual coercion also is hypothesized to function as an anticuckoldry tactic (Lalumière et al. 2005; Thornhill \& Thornhill 1992; Wilson \& Daly 1992; see also Goetz \& Shackelford 2006). Instances of forced in-pair copulation (FIPC) have been documented in avian species that form long-term pair-bonds (Bailey et al. 1978; Barash 1977; Birkhead et al. 1989; Cheng et al. 1983; Goodwin 1955; McKinney et al. 1984). FIPC is hypothesized to be a form of post-copulatory male-male competition - that is, a sperm-competition tactic (Barash 1977; Cheng et al. 1983; Lalumière et al. 2005; McKinney et al. 1984), because it often follows a female partner's extra-pair copulation or intrusions by rival males (e.g., Bailey et al. 1978; Barash 1977; Birkhead et al. 1989; Cheng et al. 1983; Goodwin 1955; McKinney et al. 1983; McKinney \& Stolen 1982; Valera et al. 2003). Sperm competition occurs when a female copulates with and is inseminated by more than one male in a sufficiently brief period of time (Parker 1970). Thus, by forcing the female to copulate shortly after the increased risk of insemination by a rival, males place their sperm in competition with any sperm deposited into their partner by a rival male (Birkhead et al. 1989; Cheng et al. 1983).

Observations of sperm competition in nonhuman species offer a framework with which to consider similar adaptations in humans, who also form long-term socially (but not genetically) monogamous pair-bonds. Recent evidence suggests that sperm competition has been a recurrent feature of human evolutionary history and that men have physiological and psychological mechanisms that may have evolved to solve related adaptive problems (Baker \& Bellis 1993; Gallup et al. 2003; Goetz et al. 2005;
Kilgallon \& Simmons 2005; Pound 2002; Shackelford \& Goetz 2007; Shackelford \& Pound 2006; Shackelford et al. 2002; 2005b; Smith 1984). It has been hypothesized that, by forcing their partners to have sex, men who are suspicious of their partner's infidelity introduce their own sperm into their partner's reproductive tract and thereby decrease the risk of cuckoldry. Thornhill and Thornhill (1992) argued that women who resist or avoid copulating with their partners might thereby be signaling to their partners a recent sexual infidelity; hence, forced copulation might function to decrease men's paternity uncertainty. And the fact that rape of a woman is more likely to occur during or after a breakup (when men's concerns about women's infidelities are greatest) may provide preliminary support for this hypothesis (see Thornhill \& Thornhill 1992). A number of studies have documented a positive relationship between men's sexual jealousy and men's sexual coercion of their partners. For example, Frieze (1983) and Gage and Hutchinson (2006) found that men who sexually coerced their wives are more sexually jealous than men who did not. Previous research has found a direct positive relationship between men's suspicions and accusations of partner infidelity and men's sexual coercion of their partners (Starratt et al. 2008). In two studies securing data from men's self-reports and women's partner-reports, Goetz and Shackelford (2006) found that men's sexual coercion correlated positively with women's past and future likelihood of engaging in sexual infidelity.

We recognize that sex differences in intimate partner violence can be explained by sex differences in reproductive strategies and by social roles, as Archer argues; however, men's partnerdirected violence can be more specifically predicted by perceived risk of female infidelity and male sexual jealousy. A large body of empirical evidence supports the hypothesis that men's partnerdirected sexual coercion and violence might sometimes be a product of evolved psychological mechanisms designed to prevent or punish female infidelity. The relevant evolved mechanisms interact with stable dispositions and situational factors to produce manifest behavior. Future research might benefit by using an evolutionary perspective to build models of intimate partner violence that include stable dispositions such as personality traits, environmental factors such as social roles, and situational factors such as perceived risk of partner infidelity.

\section{A quantitative genetic approach to understanding aggressive behavior}

doi:10.1017/S0140525X09990380

\section{Bart Kempenaers and Wolfgang Forstmeier \\ Behavioural Ecology and Evolutionary Genetics, Max Planck Institute for Ornithology, D-82305 Starnberg (Seewiesen), Germany. \\ b.kempenaers@orn.mpg.de \\ http://www.orn.mpg.de/kempenaers/abtkempenaers_en.html forstmeier@orn.mpg.de \\ http://www.orn.mpg.de/mitarbeiter/forstm.html}

Abstract: Quantitative genetic studies of human aggressive behavior only partly support the claim of social role theory that individual differences in aggressive behavior are learnt rather than innate. As to its heritable component, future studies on the genetic architecture of aggressive behavior across different contexts could shed more light on the evolutionary origins of male-female versus male-male aggression.

Archer's review explores the extent to which human sex differences in aggression can be explained by (1) sexual selection theory versus (2) social role theory. From the perspective of a behavioral ecologist and evolutionary geneticist this seems like a highly unequal comparison. While sexual selection theory 
provides ultimate explanations ("Why has it evolved?") based on principles that universally apply to the entire animal kingdom, social role theory provides a proximate explanation ("How does it come about?") that is limited to at best a small range of higher taxa.

Social role theory argues that sex differences in aggressiveness are learnt rather than innate, a proposition that may be best explored using quantitative genetic methods. The extent to which individual differences in aggressiveness (within each sex) are genetically determined, as opposed to affected by the rearing environment, should also shed some light on the relative importance of innate versus social factors that could act on the between-sex difference in aggressiveness. A large meta-analysis of genetic versus environmental influences on human aggressive and criminal behavior (Rhee \& Waldman 2002) suggests that there indeed are some effects of the family rearing environment (explaining $16 \%$ of the variance). However, this is considerably less than the joint additive and non-additive genetic effects, which explain $41 \%$ of the observed variation. Hence, humans seem to show both - a certain level of genetic polymorphism with regard to aggressive behavior (within both sexes), as well as a certain amount of behavioral flexibility allowing humans to adjust their behavior in response to their environment (i.e., the perceived costs and benefits of aggressiveness). This flexibility may underlie the observation that male aggression against female partners declines with the Gender Empowerment Index (Archer's Fig. 2), which may reflect increasing reputational costs to male perpetrators and increasing risk of retaliation by the female partner (e.g., risk of being sued, risk of being divorced).

While behavioral flexibility often seems adaptive, sexual selection theory per se does not predict whether differences in behavior will result from genetically fixed (innate) strategies or individually flexible reactions to environmental cues. However, sexual selection theory does differentiate according to the ultimate goals of aggressive behavior. Here we take issue with the definition of aggression as a "behavior intended to harm another individual" (see target article, sect. 1, para. 2). We argue that this definition emphasizes a possible - but not necessary - consequence of the behavior, instead of focusing on its aim, which is to defend or obtain a resource. Ultimately, aggression serves to secure reproductive success (Darwinian fitness), which can be achieved by securing or defending resources. Therefore, in the context of Archer's review, it seems useful to differentiate among different types of conflicts over resources that may lead to variation in sex differences in aggression. Sexual selection theory can make predictions about the following:

1. Variation in male aggression against a female partner as a form of paternity protection. Indeed, if promiscuity occurs, males may risk losing paternity (i.e., the limited resource of fertilizable eggs).

2. Variation in male sexual aggression (against partner or nonmate), including forced copulations, to obtain paternity.

3. Variation in aggression towards conspecifics of the same sex to obtain or defend resources that give access to mates (e.g., food, territory) or to obtain or defend the mate(s) themselves.

4. Variation in aggression towards heterospecific individuals to obtain or defend resources (e.g., food, nest sites).

5. Variation in aggression towards conspecifics (not necessarily sex-dependent) or towards heterospecific individuals to defend offspring (e.g., protection against predation or infanticide).

Hence, aggression can be both a component of mating effort and of parental effort. Differentiating between them seems important because they might be caused by different proximate mechanisms, and because sex differences may have different underlying causes. Interestingly, the question whether aggressive behavior is genetically correlated among different contexts remains hugely underexplored.
As to the issue of male aggression directed towards the female mate, the important question to ask (in Archer's Table 4) would have been the percentage of women versus men who avoid having an extramarital affair due to fear their husband/wife could seriously injure them if they found out. It seems possible that the evolutionary origin of violence by males against their female partners has its roots in paternity insurance (Valera et al. 2003), rather than being a byproduct (a genetic corollary) of greater male strength that evolved due to male-male competition. This could be tested by estimating the genetic correlation between male aggressiveness against their partner and aggressiveness against rival males. In any case, the fact that most women show strong preferences for tall and physically strong partners (e.g. Pawlowski \& Koziel 2002; Frederick \& Haselton 2007) suggests that over evolutionary times women benefited more from their partners' protection than they suffered from their men's physical superiority.

Further insights could be gained by considering species that show a partial sex-role reversal, as occurs in some birds or fish. In these species, male parental investment is larger than that of females, and females have a higher potential reproductive rate than males. Hence, males tend to be choosy while females compete among each other for access to males (Eens \& Pinxten 2000). Increased competition among females led to a stronger selective pressure on females to win in physical fights, and thereby to the evolution of larger and more aggressive females compared to the relatively small and peaceful males (Dale et al. 2007). One would predict that these females show hardly any aggression against their male partners, since maternity (as opposed to paternity) is never uncertain. Support for this prediction would then suggest that the larger and more competitive sex does not principally dominate the smaller and less competitive sex, but rather, that within-pair aggression by males evolved as a specific adaptation to paternity insurance. In contrast, a strong positive genetic correlation between intra- and inter-sexual aggressiveness would suggest that male aggression against the partner could arise as a genetic corollary (i.e., a possibly even maladaptive byproduct) of intense male-male competition.

\section{More holes in social roles}

\author{
doi:10.1017/S0140525X09990331
}

\section{Douglas T. Kenrick $^{\mathrm{a}}$ and Vladas Griskevicius ${ }^{\mathrm{b}}$}

${ }^{a}$ Department of Psychology, Arizona State University, Tempe, AZ, 85287-

$1104 ;{ }^{b}$ Carlson School of Management, University of Minnesota, Minneapolis, MN 55455 .

douglas.kenrick@asu.edu vladasg@umn.edu

www.carlsonschool.umn.edu/marketinginstitute/vgriskevicius

Abstract: Given the strength of Archer's case for a sexual selection account, he is too accommodating of the social roles alternative. We present data on historical changes in violent crime contradicting that perspective, and discuss recent evidence showing how an evolutionary perspective predicts sex similarities and differences responding in a flexible and functional manner to adaptively relevant triggers across different domains.

Archer argues that sex differences in within-sex aggression are better explained by sexual selection than by the alternative biosocial version of social role theory. Although Archer presents solid theoretical and empirical evidence against the social role account of sex differences in aggression, he unnecessarily pulls his punches.

Social role theorists have positioned their account of sex differences as antithetical to evolutionary psychologists' accounts. For example, in a recent exposition of their theory, Wood and Eagly 
(2007) claim: "Evolutionary psychologists have observed sex differences in modern, patriarchal societies and inappropriately concluded that humans evolved sex-typed psychological dispositions in ancestral times" (p. 389). Wood and Eagly predict a "demise of many sex differences with increasing gender equality" (p. 390). To argue that biological sex differences originate below the neck (in women's child-bearing capacities and men's greater muscular strength), and to suggest those differences would simply disappear if society treated men and women alike, is to argue for a Blank Slate.

After presenting solid evidence against the social role account of sex differences in aggression, Archer concedes that: "the similarity between the sexes that is found in post-feminist Western societies is likely to be attributable to historically recent changes in the position of women" (sect. 4.6, para. 1). But with regard to the target topic of aggression, the hardest evidence statistics on violent crime since the liberalization of sex roles in the 1970s - do not show the changes expected by sex-role theories (see our Table 1).

Cultural variations are not arbitrary. The social roles accounts debated by Archer have repeatedly taken cultural variations as evidence against an evolutionary perspective, perpetuating the misconception that universal predispositions produce phenotypic invariance in behavior across societies and across time-periods. However, numerous evolution-inspired research programs indicate that such variations can be ecologically triggered by biologically meaningful factors (e.g., Gangestad et al. 2006a; Schaller \& Murray 2008).

Consider cultural variations in age preferences (Kenrick \& Keefe 1992). Eagly and Wood (1999) argued that these stem from sex differences in social power, citing cross-cultural correlations between female power and magnitude of age preferences. Yet they fail to note that "sex differences in modern, patriarchal societies" do not disappear in non-Western societies, but instead get larger. Why are these larger sex differences in nonWestern societies? One possible explanation is linked to the fact that women in those societies have more children and therefore increase in apparent age more rapidly than women in European and North American countries. Thus, what appear to be "cultural variations" may instead be products of biologically meaningful variations in fertility cues, and not arbitrary role assignments (Kenrick \& Keefe 1992).

Young men's preferences provide one way to distinguish the two explanations. Developmental studies reveal teenage boys to be highly sex-typed. Hence, they should manifest the roletypical desire for younger, less powerful, partners. But instead, teenage boys are attracted to college-age women (Kenrick et al. 1996). This and many other findings are consistent with an attraction to fertility cues.

Tiwi society, in which young men marry older widows, seems at first glance to contradict evolutionary explanations. Is this Eagly and Wood's imagined egalitarian society, in which women have more power and patriarchy is reversed? Hardly:

Table 1 (Kenrick \& Griskevicius). Percentage of homicides committed by women. [Statistics based on FBI Uniform Crime Reports.]

\footnotetext{
$1961-1965$

$1966-1970$

1971-1975

1976-1980

$1981-1985$

1986-1990

1991-1995

1996-2000

2001-2007
}

Tiwi society is highly patriarchal and polygynous, and Tiwi women have little say in who they marry. Instead, Tiwi patriarchs enforce a rule that all females (but not all males) must be married. The elder patriarchs marry all the young females, and are not interested in older widows. By marrying an elder widow, a young man acquires rights to determine who her young daughters remarry, and establishes himself as a potential recipient of a younger wife (Hart \& Pillig 1960). So, even in a society where one aspect of typical sex-specific behavior is reversed by socially enforced caveat, evolved mating preferences do not reverse at all (Kenrick et al. 2010).

Evolutionary hypotheses are based on a comparative nomological network. A misconception advanced by Eagly and Wood and other critics is that evolutionary models depend on hard-to-observe behaviors among ancestral humans. In reality, sexual selection models, as Archer makes clear, are derived not from observations of Western patriarchal societies alone but from an immense comparative literature on still-living species. This poses a problem for sex-roles explanations, since many sex differences found among humans in Western societies are shared not only with people in other societies, but with all other mammals, and most other vertebrates. As Archer notes, when sex-role reversals are found in some species, they further support the clear link between parental investment and sexual selection.

Evolutionary psychology examines functional responses to adaptive contexts. As Archer notes, an evolutionary approach to human behavior derives hypotheses from comparative and cross-cultural data, not from observations of Western patriarchal society. From this powerful nomological network of research and theory, evolutionary psychologists derive hypotheses about how behavior varies flexibly - and functionally - in response to adaptively relevant environmental triggers. Consistent with Archer's analysis of sexual selection and aggression, activating status motives leads men but not women to desire to aggress in a
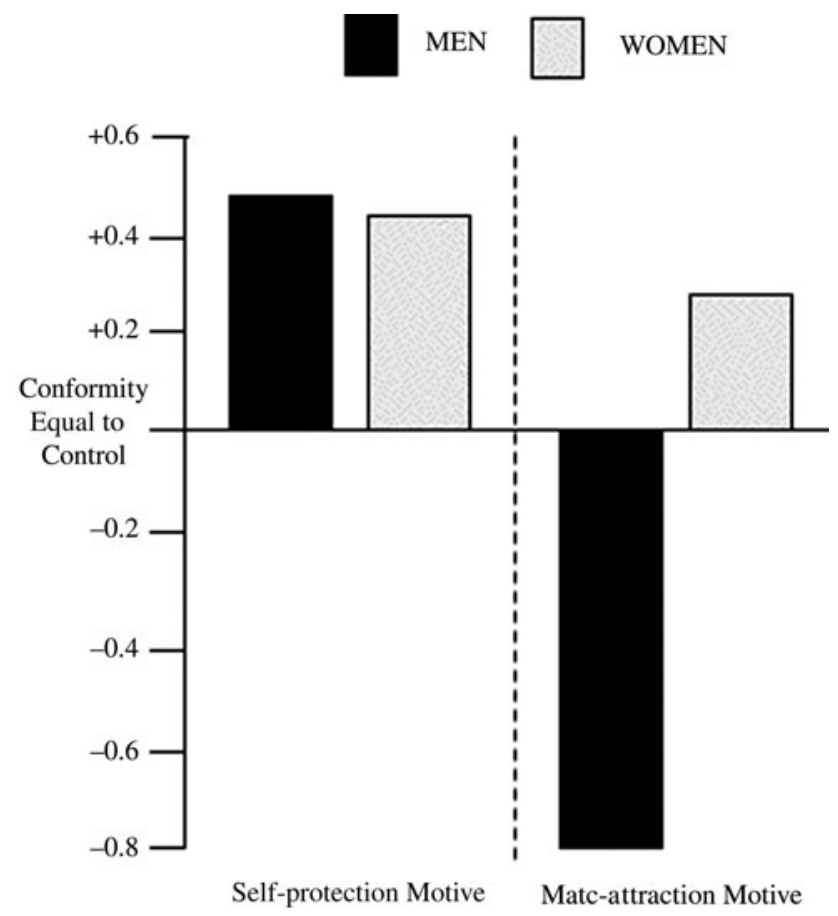

Figure 1 (Kenrick \& Griskevicius). Self-protection and mating motives have distinct consequences for conformity behaviors of men and women. Both men and women tend to be more conforming when feeling threat. Activating mating motives leads men to become less conforming and, as discussed in the text, more creative and showy in other ways. (Adapted from Griskevicius et al. 2006b) 
direct manner (face-to-face confrontation). And consistent with other domain-sensitive evolutionary accounts, resource threat leads women to respond in a manner more similar to men (Griskevicius et al. 2009). Ultimately, aggression for both men and women appears to function as a tactic for enhancing reproductive success.

Men and women also show sex-specific and functionally strategic differences and similarities when different goals are activated. For example, consistent with theories of sexual selection and differential parental investment, mating motives lead men - but not women - to engage in conspicuous, attentionattracting, displays, including conspicuous consumption and creative flair (Griskevicius et al. 2006a; 2007). Such findings are not merely demonstrations of arousal enhancing sex-role typical behavior. For instance, activating mating motives leads men to go against group opinion, whereas mating motives lead women to conform more (Griskevicius et al. 2006b). However, activating self-protective motives eliciting similar levels of arousal do not lead men to act in "macho" ways as might be expected. Instead, self-protective motives lead both men and women to become more conforming and group-oriented (see our Fig. 1).

Research generated from an evolutionary-psychological perspective is not designed to test hypotheses about historical evolution (a persistent misconception), but to consider proximate causes of human behavior in ways consistent with functional analyses. This approach has generated abundant evidence showing that men and women do not randomly remember and then forget their sex-roles, but instead respond in adaptively flexible ways to functionally relevant environmental cues (Griskevicius et al. 2009; Kenrick \& Sheets 1993). Archer's target article does an excellent job dispelling some of these misconceptions, pointing to strong theoretical and empirical evidence supporting a sexual selection account of sex differences in within-sex aggression.

\section{Moderators of sex differences in sexual selection theory}

\section{doi:10.1017/S0140525X09990379}

\section{Anthony D. Pellegrini}

Department of Educational Psychology, University of Minnesota, Twin Cities Campus, Minneapolis, MN 55405.

pelle013@umn.edu

\begin{abstract}
Archer recognizes that sexual selection theory is sensitive to the effects of ecologies on sex differences, yet he does not explain the impact of such variation. For example, to what degree are there sex differences in aggression in polygynous and monogamous societies? I demonstrate how differences in mating perceptions affect the traditional dichotomy that males compete for and females choose mates.
\end{abstract}

Archer's review is an important addition to the continuing debate around sex differences in human behavior, generally, and aggression, more specifically. Concerning the wider debate, a recent review (Hyde 2005) minimized the importance of sex differences in human behavior. One dimension of that argument was based on tallying the number of studies showing sex differences in relation to those not showing differences. That method presents an interesting issue: Do we judge the importance of differences based on the number of differences tallied across all observed behavior and traits, or should judgments be guided by testing for specific differences specified by a theory where differences between certain behaviors and traits are more important than others? My bias is with the latter approach.

Archer also takes this tack by evaluating the extant literature in relation to hypotheses for sex differences in aggression generated by Darwin's (1871/1901) sexual selection theory and social role theory (Eagly 1997). This is a debate with a lively and healthy history; the very sort of debate that helps advance science. Focus on the degree to which aggression is intersexual, or primarily directed on members of the other sex, specifically males aggressing against females, or intrasexual, male-male or femalefemale, is crucial to the debate. Traditional versions of sexual selection theory (Darwin 1871/1901) as well as revised versions (e.g., Clutton-Brock 2009) posit that males' and females' competition should be primarily intrasexual; and that males, more than females, should compete with each other for mates using direct aggression, and females compete less directly with each other for resources. That within-sex aggression is greater than that between sexes, even among preschoolers (Pellegrini et al. 2007b) and middle school youngsters (Pellegrini \& Long 2003), and among adults, as shown in this review, highlights the robustness of the finding.

Recently scholars have begun to recognize the importance of females' intrasexual competition (e.g., Campbell 1999), and this has lead to the reformulation of sexual selection theory (Carranza 2009; Clutton-Brock 2009). In contrast to males, females' intrasexual competition should be aimed at accessing and maintaining resources related to raising and provisioning offspring and should result in the selection of a phenotype that maximizes social support, in the forms of alliance formation, manipulation, and indirect aggression (Hrdy 2005).

An important aspect of this reformulation is that the expression of any behavior is moderated by the ecological niche in which individuals are embedded, a position consistent with both Darwin (1871/1901) and his predecessor John Hunter (Hunter 1780; cf. Clutton-Brock 2009). This is not to say that phylogenetic history is irrelevant. Instead, this history probably canalizes (Hinde \& Stevenson-Hinde 1973) the behaviors and strategies to be learned (during ontogeny) in the context of social groupings (Crook 1989). Variations in different ecologies, in turn, result in individuals' developing alternative strategies in the course of ontogeny (Caro \& Bateson 1986). Experiences early in ontogeny alert the organism to the environment in which they will function so as to maximize their adaptation to that niche. Different phenotypes develop during childhood in sexually segregated groups during childhood, to prepare boys and girls for adult roles. Briefly, ecologies labeled as "abundant" (Bateson \& Martin 1999) should be characterized by polygynous mating patterns and males, relative to females, being more competitive and active. This high level of activity should result in segregated juvenile groups with males being characterized by physically activity and aggressive behavior and females by more sedentary and nurturing behavior (Pellegrini 2004). More severe ecologies should witness more "thrifty phenotypes" (Bateson \& Martin 1999), resulting in monogamous or cooperative breeding and less difference between the sexes in competition and activity. Consequently, peer groups should be less segregated, resulting in fewer sex differences in aggression. Archer himself acknowledges the role of such variation, yet he does not go into sufficient detail to explain variation in different ecologies. For example, sexual selection theory predicts that sex differences in size and aggression should be most pronounced in polygynous societies. To what degree does this occur?

Traditional sexual selection theory also posits that more intense male-male competition should result in corresponding sex differences in body size, with males being physically larger than females. This review, however, has not explained the reliable sex difference is physical aggression during early and middle childhood when there are no differences in dimorphism for physical size. Why is that? One argument holds that the sex differences in physical activity (Pellegrini et al. 2007a) and locomotor play (Pellegrini \& Smith 1998) during early childhood reflect the differences necessary to shape males' and females' skeletal and muscular systems that differ in adulthood.

Further, contextual variation associated with males' uses of within-sex aggression to attract mates within an evolutionary 
framework has been addressed in a recent and very provocative paper by Buston and Emlen (2003). They suggest that mate choice, at least among Cornell undergraduates, is not governed by mate "potential" attributes - for example, attractive women choosing strong, high-status males, and strong, high status men choosing attractive women. Instead, they found that individuals choices were based on their perceptions of the possible duration of the relationships. When there was expectation of a long term, stable relationship, individuals chose mates similar to themselves (on factors such as physical attractiveness, status, and commitment to family). Attractive women chose attractive men; they did not choose strong, aggressive men. Situations in which costs associated with mate switching is high, for example, where divorce is economically disruptive, would result in individuals choosing mates who are similar to themselves (see Borgerhoff Mulder 2004).

By contrast, aggressive men (again, where aggression is intrasexual) were chosen by physically attractive women in situations in which the quality of available mates is low. In this latter case, male-male aggression would be reinforced by female choice and females would "sample" widely from available males for a strong mate who also protects and provisions her and her offspring.

With these limitations stated, Archer's target article makes an important contribution toward the theoretical integration of a wide and disparate literature. He has done the field an important service.

\section{There's no contest: Human sex differences are sexually selected}

\section{doi:10.1017/S0140525X0999032X}

\section{Nicholas Pound, ${ }^{\mathrm{a}}$ Martin Daly, ${ }^{\mathrm{b}}$ and Margo Wilson ${ }^{\mathrm{b}}$ \\ ${ }^{a}$ Department of Psychology, School of Social Sciences, Brunel University, Uxbridge UB8 3PH, United Kingdom; ${ }^{\mathrm{b}}$ Department of Psychology, Neuroscience, and Behaviour (PNB), McMaster University, Hamilton, Ontario L8S 4K1, Canada. \\ nicholas.pound@brunel.ac.uk \\ daly@mcmaster.ca \\ wilson@mcmaster.ca}

Abstract: An evolutionary psychological perspective drawing on sexual selection theory can better explain sex differences in aggression and violence than can social constructionist theories. Moreover, there is accumulating evidence that, in accordance with predictions derived from sexual selection theory, men modulate their willingness to engage in risky and violent confrontations in response to cues to fitness variance and future prospects.

In the target article, Archer argues persuasively that an evolutionary psychological perspective drawing on sexual selection theory can account for observed sex differences in aggression and violence more parsimoniously than social constructionist theories. In our view, however, the case for sexual selection's role in the evolution of these sex differences is even stronger than Archer's treatment suggests, and he concedes too much to advocates of the discredited null hypothesis that female and male psyches are undifferentiated.

According to Archer (sect. 2.2.1), the "main alternative" to a selectionist explanation of the origins of the sex differences of interest is the "social role" theory of Eagly and Wood (1999; Wood \& Eagly 2002). At best, these authors can be read as offering a partial account of ontogenetic processes in sexual differentiation, which, if upheld, would complement an evolutionary account. At worst, they can be read as proposing that the only evolved differences between women and men are "physical" (i.e., non-neural anatomical differences), and if this is indeed their meaning, they are simply uninformed (see, e.g., Kimura
1999). In neither case have they provided a viable "alternative" to an account that gives centre stage to sexual selection.

In explaining why sexual selection should have made men more intensely competitive than women, Archer (sect. 3.2) aptly cites anatomical, demographic, and behavioural evidence that Homo sapiens evolved as an effectively polygynous species in which male fitness variance exceeded female fitness variance. Recent genetic evidence (Wilder et al. 2004) reinforces this conclusion: In both our species as a whole and in discrete subpopulations thereof, the most recent common ancestor (MRCA) of mitochondrial DNA, inherited matrilineally, lived about twice as long ago as the MRCA of Y chromosomes, inherited patrilineally. These results provide strong evidence that individual men have consistently faced a higher risk of reproductive failure than individual women.

Archer notes (sect. 2.8) that, consistent with sexual selection theory, there is evidence that males with limited access to reproductive opportunities, or the resources required to obtain such opportunities, are more likely to resort to violence. However, there is accumulating evidence to support a more specific prediction derived from the same theoretical perspective, namely that the prevalence of dangerous confrontations should vary predictably according to variations in the local intensity of intrasexual competition and that cues to higher fitness variance should lead males to modulate their willingness to engage in risky and violent interactions with other men (for review, see Wilson et al. 2002; 2009).

In an effectively polygynous species, the intensity of male-male competition will in part depend on the extent to which the resources required to obtain reproductive opportunities are distributed equitably. Extreme inequity creates a situation where those at the bottom have little to lose if they escalate their tactics of competition, and much to gain. Consequently, cues to inequity should lead to facultative adjustments in men's willingness to employ violent and risky tactics to gain status and resources, which are the means to fitness. Consistent with this, evidence indicates that relative deprivation (as indexed by income inequality) is typically a more powerful predictor of variation in male violence than other socioeconomic measures such as percent below the poverty line or average income (Daly \& Wilson 2001). In both cross-national and more local comparisons, the Gini index of income inequality consistently outperforms most other socioeconomic predictors of homicide rates (e.g. Blau \& Blau 1982; Daly et al. 2001).

Increased willingness to resort to violence where resources are distributed inequitably is not uniquely predicted by sexual selection theory. However, in contrast to social constructionist accounts, an evolutionary psychological perspective treats such increased risk-proneness as a facultative adaptive response to situations where the distribution of resources is such that excessive risk-aversion will lead to substantially reduced expected fitness (Wilson et al. 2002; 2009).

An evolutionary psychological approach based on sexual selection and life history theory also predicts that individuals should modulate their willingness to engage in risky and violent confrontations according to cues of future survival and hence reproductive prospects - in other words, when prospects are poor, organisms may be expected to discount the future steeply in the pursuit of more immediate goals (Daly \& Wilson 2005). Archer notes (sect. 3.1) that greater male than female mortality is characteristic of the sexually selected "adaptive complex" generated by intense inter-male competition, but the target article could perhaps have examined the implications of this in more detail. It is not just that males are likely to discount the future more heavily than females as a consequence of the sex difference in mortality; moreover, future discounting and willingness to engage in risky escalation of social conflicts are expected to vary predictably in relation to future survival prospects. Where these are poor, men should become particularly risk prone and willing to risk death in violent altercations as they compete for 
the resources required to obtain reproductive opportunities, and directly for the opportunities themselves. Consistent with this, Wilson and Daly (1997) found that across neighbourhoods in a major U.S. city (Chicago), the best statistical predictor of homicide rates was low male life expectancy (even with homicide as a cause of death removed).

Finally, we do not understand Archer's rationale for suggesting that a sexual selection approach warrants the "prediction" that the sexes will not differ in "aggression" or "anger," but only in how they manifest these things. Sell's research and theorizing (e.g., Sell et al. 2005), which Archer cites, clearly suggests that insofar as becoming angry entails an elevated risk of violent confrontation, we may expect people to differ adaptively in their readiness to anger. Why should this proposition not apply to sex differences? More fundamentally, what does it even mean to suggest that men and women do not differ in "aggression" or "anger," but only in the manifestations thereof? We lack both consensual definitions and good metrics of these states, and finding that the sexes give the same mean answer on a selfreport scale of "aggression" or "anger" is uninformative. Consequently, evidence for the popular claim that men and women are equally aggressive, but that the former manifest their aggression "directly" (e.g., as violence) and the latter "indirectly" (e.g., as innuendo) is not convincing.

\section{Sex differences in dream aggression}

doi:10.1017/S0140525X09990306

\section{Michael Schredl \\ Sleep Laboratory, Central Institute of Mental Health, J5, 68159 Mannheim, Germany. \\ Michael.Schredl@zi-mannheim.de \\ www.dreamresearch.de}

Abstract: Dream research shows sex differences in dream aggression that fit very well with the findings for waking-life aggressive behaviour. Dream studies are a valuable tool for investigating variables underlying the sex difference in aggression. One might argue that studying dream aggression might be even more promising because aggression in dreams is not socially labelled, as being aggressive in waking life is.

Since dreams reflect waking life experiences (the so-called continuity hypothesis of dreaming; Schredl 2003), dream studies can elucidate sex differences reported for waking behaviour. For example, it is a stable finding that men report more sexual dreams than do women (Schredl et al. 1998), which reflects the meta-analytic findings of higher frequency of masturbation and sexual fantasies in males compared to females (Oliver \& Hyde 1993). Regarding aggression in dreams, the findings are in line with the meta-analysis reported by Archer: Men's dreams included more physical aggression than women's dreams did (Hall \& Van de Castle 1966), whereas the amount of verbal aggression did not differ between the sexes (Schredl et al. 1998). The gender difference regarding the percentage of physical aggression (50\% in men's dreams vs. 34\% in women's dreams; Hall \& Van de Castle 1966) is quite stable over time. The data collection period of Hall and Van de Castle (1966) ranged from the late 1940 s to the early '50s. Subsequent studies in the '70s (Hall et al. 1982) and '90s (Schredl et al. 2003), and very recent studies (Schredl \& Keller 2008-2009) replicated the higher prevalence of physical aggression in men's dreams compared with total aggression. This means that cultural developments such as the women's movement did not affect this sex difference in dreams. And this favours the sexual selection theory over the social role theory.
Another aspect of dream aggression fits the theory put forward by Archer: the higher difference in the aggression per male character compared to the aggression per female character in men ( 0.23 vs. 0.13 for men, 0.13 vs. 0.10 for women). That is, women experience aggressive interactions with both men and women in almost equal frequency in their dreams, whereas men's dreams include same-sex aggression more often compared to opposite-sex aggression (Hall \& Domhoff 1963).

Also very interesting is the shift in the percentage of physical aggression over the life-span. Whereas children (younger than 11 years old) showed the typical gender difference of more physical aggression in boy's dreams compared with girl's dreams, the ratio of physical aggression in dreams is the same for both sexes in the age range from 12 to 17 years (Hall \& Domhoff 1963; Oberst et al. 2005). This fits with Archer's argument that males avoid risky encounters with older males prior to adulthood. The adults - as reported above - again showed the preponderance of physical aggression in men's dreams.

Domhoff (1996) reviews cross-cultural dreams studies. Whereas many Western countries showed higher prevalence rates of dream aggression in men compared to women, there where several exceptions. The Hopi Indians, for example, showed no gender differences in overall aggression and in the percentage of physical aggression (Domhoff 1996). The term Hopi can be translated into "peaceful ones" reflecting the lifestyle of these Pueblo Native Americans. But for some industrial countries, such as Switzerland and Japan, the ratios of physical dream aggression were not different between the sexes. This indicates that cultural factors modulate the amount of aggression in dream. In females, dream aggression was more often found in dreams of non-traditional women, indicating again the cultural effect on aggression pointed out by Archer. Unfortunately, these studies did not differentiate between same-sex aggression and opposite-sex aggression to enable us to test Archer's claim that cultural factors might be more important in explaining the amount of opposite-sex aggression.

Two studies, by Waterman et al. (1988) and Cohen (1973), investigated whether biological sex or feminine versus masculine sex role orientation explains differences in dream aggression. Whereas the finding of the first study was unambiguous (only biological sex was of importance), the second study showed effects of both variables on the amount of dream aggression. Again, it would have been fruitful to differentiate between same-sex and opposite-sex aggression.

Another interesting gender difference can be found in the bad dreams and nightmares of children (Schredl \& Pallmer 1998). In Table 1, the percentages of male and female aggressors are presented.

Male characters threaten the dreamer most often whereas women are quite rarely aggressors in dreams. The ratio of male and female aggressors in dreams is similar for boys and girls, thus reflecting the preponderance of male aggression in mass media (news, films, etc.). It would be very interesting to study the gender of the aggressor in cross-cultural dream studies in more detail, for example, in societies without predominance of male aggression. If dream aggressors are still mostly male, one

Table 1 (Schredl). Human aggressors in children's dreams

\begin{tabular}{llll}
\hline \hline Aggressors & $\begin{array}{l}\text { Total } \\
(\mathrm{n}=111)\end{array}$ & $\begin{array}{l}\text { Boys } \\
(\mathrm{n}=35)\end{array}$ & \multicolumn{1}{l}{$\begin{array}{l}\text { Girls } \\
(\mathrm{n}=76)\end{array}$} \\
\hline Male (unfamiliar) & $49.7 \%$ & $51.4 \%$ & $48.7 \%$ \\
Female (unfamiliar) & $3.6 \%$ & $0.0 \%$ & $5.3 \%$ \\
Male (familiar) & $27.8 \%$ & $31.4 \%$ & $26.2 \%$ \\
Female (familiar) & $18.9 \%$ & $17.2 \%$ & $19.8 \%$ \\
\hline \hline
\end{tabular}


might argue that gender differences in aggression have hereditary aspects.

To summarize, dream studies seem to be a valuable tool for studying sex differences in aggression, because the findings fit very well with the findings for waking-life aggressive behaviour. One might argue that studying dream aggression might be even more promising because aggression in dreams is not labelled socially the way being aggressive in waking life is. This is supported by the fact that aggression, even physical aggression, is quite common in dreams (Hall \& Van de Castle 1966). For future studies, it would be very interesting to study the effect of mediator variables mentioned by Archer on the amount of dream aggression - for instance, the effects of empathy, risk-taking behaviour, and assertiveness, as well as physiological measures such as testosterone levels, size, and strength.

\section{Human sexual dimorphism, fitness display, and ovulatory cycle effects}

\section{doi:10.1017/S0140525X09990240}

\section{Jon A. Sefcek ${ }^{\mathrm{a}}$ and Donald F. Sacco ${ }^{\mathrm{b}}$ \\ ${ }^{a}$ Department of Psychology, University of Arizona, Tucson, AZ 85721-0068; \\ ${ }^{\mathrm{b}}$ Department of Psychology, Miami University, Oxford, OH 45056. \\ lennonjon@gmail.com saccodf@muohio.edu \\ http://www.u.arizona.edu/ jons}

Abstract: Social roles theorists claim that differences between the sexes are of limited consequence. Such misperceptions lead to misunderstanding the important role of sexual selection in explaining phenotypic differences both between species and within humans. Countering these claims, we explain how sexual dimorphism in humans affect expressions of artistic display and patterns of male and female aggression across the ovulatory cycle.

We would like to applaud Archer for his target article, "Does sexual selection explain human sex differences in aggression?" The tone of the article title is reminiscent of the November 2004 National Geographic cover article that asked: "Was Darwin Wrong?" (With a resounding "NO!" answering the reader on the opening page). It's no surprise that Archer has confidently proclaimed a resounding "YES!" to his own question.

Archer effectively argues that social role theory fails to adequately explain the complexity of aggressive patterns found both within humans and across species by requiring that its adherents downplay the dramatic similarities that permeate the animal kingdom, underestimate cross-cultural similarities in human aggressive behavior, and disregard the impact of human sexual dimorphism. Here we would like to extend Archer's critique of social role theory by looking at differences both between animal species and the human sexes.

According to social role, or biosocial, theory (e.g., Eagly \& Wood 1999; Wood \& Eagly 2002), sex differences in social behavior result from the distribution of men and women into different social roles within society. These differences stem from sex differences in the physical attributes of the sexes as well as the changing local ecologies of humans. Despite acknowledging these dimorphisms as the starting point, this framework reduces the "role" of sexual selection in shaping these differences to little more than a historical footnote by failing to attend adequately to the variety of evolutionary forces integral in shaping the degree of sexual dimorphism within species.

Largely due to parental investment and mating patterns, which themselves are largely determined by the local ecological conditions, dimorphisms may take the shape of physical attributes such as body size, or behavioral attributes such as behavioral fitness display (see Sefcek et al. 2006, for review). Specifically, social role theory argues that hominid sexual dimorphism is small in magnitude compared to other primates and driven as much by fluctuations in female body size as it is by intrasexual competition (Plavcan \& van Schaik 1997b). We argue that the less-extreme dimorphism in humans is not evidence that the dimorphisms are not significant. Although the human sexes, which are approximately $15-20 \%$ different in body size, do not show the extreme dimorphisms of male and female gorillas or orangutans (e.g., these males being $50 \%$ larger in body size), they also do not show the remarkable similarity (monomorphism) in body size that gibbons and other socially monogamous primates do (Leutenegger 1982). That humans fall somewhere in-between suggests at least a moderate level of dimorphism as the result of a species-typical evolutionary history of moderate polygyny, cuckoldry, male parental investment, and male intrasexual competition, which leads to sex-typical differences in mating effort - that may manifest itself through aggression and other risky behaviors (Sefcek et al. 2006).

One important difference in mating effort is illustrated through sex differences in fitness indicating, whereby an individual "shows-off" in an effort to display behavioral energy or genetic quality (Miller 2000). Such ornamental display is risky; it redirects metabolic resources away from survival and makes the individual conspicuous to intrasexual rivals and interspecies predators. Such advertisement may, however, yield a highpayoff. While humans do not display extreme physical ornaments like the peacocks tail, it is argued many products of the human mind and culture serve this fitness signaling function. Cultural artifacts produced through artistic expression (e.g., painting, musical production, poetic language, and humor) and scientific discovery are male-biased and are suggested to stem from intrasexual competition (Kanazawa 2000; Miller 2001). These patterns tend to show male-biased sexual dimorphism in public display, yet monomorphism in both public appreciation and private display.

Furthermore, both men and women display cycle-specific dimorphic perceptual and behavioral changes related to aggression and self-protection that are in the service of enhancing reproductive fitness. For example, men perceive more dominance in male faces when their partner is ovulating, suggesting an evolved bias to identify the most likely intrasexual competitors in order to protect against potential cuckoldry (Burriss \& Little 2006). Fertile females who rate their partners as sexually unattractive (a phenotypic sign of high mutation load) report greater extrapair flirtation and more partnerenacted mate guarding behavior, itself an aggression-based mating tactic (Haselton \& Gangestad 2006). Finally, men report more use of sexual coercion in intimate relationships when they perceive that their partner has engaged in sexual infidelity (Goetz \& Shackelford 2006), as well as report deeper and more vigorous penile thrusting (Gallup et al. 2003), and a higher sperm count per ejaculate (Baker \& Bellis 1995) after periods of separation or alleged sexual infidelity. Conversely, fertile females become less aggressive and even though they report more attraction to male dominance, they engage in fewer risky behaviors - which suggests that women strategically avoid the types of activities that may increase their exposure to male aggression when fertile (Broder \& Hohmann 2003). Furthermore, in response to sexually coercive scenarios, female handgrip strength increases at peak ovulation, an adaptation that may have evolved to protect females against sexually coercive male tactics (Petralia \& Gallup 2002).

That so much variability in men's aggressive behaviors and female's self-defense behaviors is contingent on fluctuations in female fertility and potential infidelity, strongly suggests that human aggression in its various forms may be better explained as solutions to reproductive problems, shaped via sexual selection, rather than simply as consequences of social roles. Although it is certainly the case that men and women often respond to the same cues with aggression, the fact that aggression, fertility, and 
perceptions of infidelity are so intertwined suggests that sex differences in aggression are rooted in our evolutionary past. Because men and women, by dint of their biology have historically faced different adaptive challenges to reproductive success, those who inherited the most effective sexual strategies (e.g., Buss 1998) would be better equipped to effectively reproduce. It seems that one strategy for differentially improving reproduction includes sex-specific risky-behavioral tendencies, with women focusing on low-risk forms of aggression and males focusing on high-risk forms of aggression.

\section{Standards of evidence for designed sex differences}

doi:10.1017/S0140525X09990276

\section{Aaron Sell \\ Department of Psychology, University of California, Santa Barbara, Santa Barbara, CA 93106-9660. \\ sell@psych.ucsb.edu \\ http://www.psych.ucsb.edu/research/cep/grads/Sell.html}

\begin{abstract}
At the heart of the debate between social role theorists and evolutionary psychologists is whether natural selection has designed the minds of the sexes differently to some interesting extent. In this commentary I describe the standards of evidence for both the positive and negative claims. In my opinion, Archer has met the standard for designed sex differences in intrasexual conflict.
\end{abstract}

George Williams argued that natural selection results in three categories of features: adaptations, by-products and noise (Williams 1966). The same classifications hold for sex differences, and the debate between evolutionary psychologists and gender role theorists can be understood as a debate about which of two categories sex differences in aggression falls into. Because Williams was classifying features and not differences in features, some slight translation is necessary.

Evolutionary psychologists tend to think that sex differences in aggression are adaptive differences; that is, they are sex differences resulting from adaptations designed differently in men and women by natural selection in response to differing ancestral selection pressures. Non-controversial examples include sex differences in body size and maturation rates. Gender role theorists tend to think of sex differences in aggression as learned byproducts, that is, as sex differences that result from learning mechanisms (which were designed by natural selection, of course) that are the same in men and women but create differences because of differential input. Non-controversial examples of this would be sex differences in car seat settings and fear of prostate cancer. Finally, there are arbitrary differences: these are sex differences that result from accidents of history and are not designed by natural selection but also do not stem from learning mechanisms in ways that lead to sensible outcomes. Non-controversial examples would be sex differences in styles of dress, the spelling of names (e.g., Aaron vs. Erin), and culturally agreed upon color symbols (e.g., pink for girls, blue for boys).

The standard of proof for adaptive differences is parallel to those for adaptations. One has to show evidence of complex functional design, geared toward solving an adaptive problem that acted differently on the sexes. Archer has done this for sex differences in intrasexual aggression. He lays out a complex of features, all of which would result from a differential selection pressure (namely, sexual selection). They include, for males: greater variance in reproductive success, greater size and strength, longer maturation times, higher mortality and male-biased conception ratio. I would add that physical differences in size and strength are also supplemented by sex differences in basal metabolic rates (Garn \& Clark 1953), heart size, heat dissipation, hemoglobin, muscle-to-fat ratio, and bone density (Lassek \& Gaulin, in press). Across all those variables the sex difference is in the direction of males being designed for physical aggression. Additionally, boys prefer rough-and-tumble play, a type of activity that is understood by evolutionary biologists to be practice for future combat (Symons 1978). This last point is particularly important because of the overwhelming evidence that sex differences in rough-and-tumble play are not caused by societal expectation. Girls born with congenital adrenal hyperplasia (CAH) as well as progestin-induced hermaphrodism (PIH) are typically raised as girls, and genetically are girls, but experience some heightened organizing effects of androgens during development. As a result, they engage in more boy-like play patterns, including rough-andtumble play (Daly \& Wilson 1983). All of these coordinated features, each in the predicted direction, provide powerful evidence that natural selection designed males and females differently when it comes to aggressive tendencies.

The evidence required to put sexual differences in aggression in the category of learned byproducts is as follows: (1) identify the adaptation (or adaptations) that aggressive differences result from, and (2) demonstrate why, as a byproduct of their design, those adaptations would lead to sex differences. Gender role theorists have taken steps in these directions, specifying that evolved physical differences in strength and size coupled with a cost-benefit analysis mechanism (and more traditional socialization mechanisms) will produce differences in aggressive tendencies (Wood \& Eagly 2002). As Archer points out, however, the data are stacked against this theory. He mentions that aggression's developmental trajectories are inconsistent with socialization theory, and the role of testosterone and operational sex ratio are difficult for gender theorists to explain. The data from $\mathrm{CAH}$ and PIH girls also contradicts the idea that gender roles lead to differential aggression, as the girls generally maintain a female identity even while increasing their aggressive play. Finally, from a theoretical point of view, one has to ask why natural selection would have selected genes that created sex differences in body size and strength if males and females were aggressing at equal rates.

With regard to intersexual aggression, it is important to keep in mind that similarities between men and women on broad measures of aggression can hide sex differences in particulars. For example, men and women have similar rates of spousal homicide that are motivated by sexual jealousy, but it was male jealousy that resulted in both the killing of husbands, usually in defense, and wives, usually out of jealous anger (Daly \& Wilson 1988). As Archer says, both evolutionary and social role perspectives predict that spousal aggression should vary with the relative power of the individuals. The question remains, however: Which perspective will be most useful for predicting and explaining currently unknown features of mate-directed aggression? By studying selection pressures and how they work, evolutionary biologists have been able to account not only for the origin of biological complexity, but the origin of sexual reproduction itself (Tooby 1982), the origin of sexes (Parker et al. 1972), the existence of maturation and senescence (Williams 1957), the origin of aggression (Maynard Smith \& Price 1973), its major causes (Huntingford \& Turner 1987), the existence and function of aggressive displays (Alcock 1998), the magnitude and constituent features of sexual dimorphism (Daly \& Wilson 1983), and sexual differences in aggressive tendencies, homicide (Daly \& Wilson 1988), and rough-and-tumble play (Symons 1978). Finally there is a massive amount of primatological data showing how natural selection has designed males and females of other species in ways consistent with the differing adaptive problems they faced ancestrally (Smuts et al. 1987). With all that in mind, it seems likely that natural selection played some important role in the differential design of male and female minds, particularly in domains defined by differential selection pressures such as aggression. 


\section{Sex differences in human aggression: The interaction between early developmental and later activational testosterone}

\section{doi:10.1017/S0140525X09990367}

\author{
David Terburg, ${ }^{a}$ Jiska S. Peper, ${ }^{a}$ Barak Morgan,, ${ }^{b}$ and \\ Jack van Honk ${ }^{a}$ \\ ${ }^{a}$ Department of Psychology, Utrecht University, 3584 CS Utrecht, The \\ Netherlands; ' ${ }^{b}$ Department of Human Biology, University of Cape Town, \\ Department of Human Biology, Cape Town, South Africa 7925. \\ d.terburg@uu.nl j.s.peper@uu.nl barak.morgan@uct.ac.za \\ j.vanhonk@uu.nl
}

\begin{abstract}
The relation between testosterone levels and aggressive behavior is well established. From an evolutionary viewpoint, testosterone can explain at least part of the sex differences found in aggressive behavior. This explanation, however, is mediated by factors such as prenatal testosterone levels and basal levels of cortisol. Especially regarding sex differences in aggression during adolescence, these mediators have great influence. Based on developmental brain structure research we argue that sex differences in aggression have a pre-pubertal origin and are maintained during adolescence. Evidence of prenatal, adolescent, and adult levels of testosterone in relation to aggression taken together, support Archer's argument for sexual selection as the driver of sex differences in aggression.
\end{abstract}

John Archer makes a strong argument for an evolutionary basis of sex differences in aggression. His thesis is that, starting in early childhood, sex differences in aggressive behavior exist, and, although these are mediated by social influences, they are underlain by biological variables. One of these variables is the steroid hormone testosterone. Archer's conclusions regarding testosterone and adolescence need some refinement.

As Archer himself points out, data of self-reported aggression in male adolescents do not support findings in testosterone administration studies on aggression. He argues that, although exogenous testosterone seems to enhance proneness to aggression, the rising levels of testosterone in male adolescents are not reflected in self-reported aggression measures. However, behavioral studies suggest that testosterone is a mediator of adolescent aggression. James Dabbs and colleagues showed repeatedly, in a line of studies in the nineties, associations of testosterone and violent criminal behavior. Imprisoned young males with high salivary testosterone were substantially more frequently convicted for aggressive crimes like violence and rape, and they showed more violent behavior (Dabbs et al. 1995). This was also replicated in studies on women (Dabbs \& Hargrove 1997). Interestingly, in late adolescent males the hormone cortisol mediated the correlation between testosterone and aggressive behavior, which was found only in imprisoned adolescents with low cortisol levels (Dabbs \& Jurkovic 1991).

More recently, Popma and colleagues (Popma et al. 2007) showed a correlation of testosterone and self-reported measures of violent behavior, but again, this was mediated by cortisol. Designed to investigate the relation between testosterone, cortisol, and aggression in early adolescence, their study pointed to an effect of testosterone on overt aggression only when cortisol levels were low. Confirming this, Hermans et al. (2008) found in an fMRI study increased activity in the hypothalamus, amygdala, and orbitofrontal cortex in response to angry facial expressions. This network of brain structures is considered vital in human reactive aggression. Importantly, activity in the subcortical part of this network, namely, the hypothalamus and amygdala, in response to angry faces proved to be related to the ratio between testosterone and cortisol.

Based on these findings one should consider taking basal levels of cortisol into account when comparing groups on aggressive behavior. Especially during adolescence, a highly stressful period (as demonstrated hormonally by marked increases in HPA-activity [Gunnar et al. 2009] and, behaviorally, by the onset of several stress-related psychiatric illnesses [Paus et al. 2008]), the rise of testosterone levels (alone) in boys relative to girls will not necessarily result in a relative increase of aggressive behavior.

Another issue of consideration is testosterone in early development. Bailey and Hurd (2005), for instance, have shown that prenatal levels of testosterone, reflected in the 2D:4D digit length ratio, may mediate testosterone-aggression relationships. In males, higher prenatal testosterone levels correlated with physical aggression in adulthood. Interestingly, recent evidence from testosterone administration research in humans suggests that high prenatal testosterone levels increase sensitivity to behavioral effects of testosterone in later life (van Honk 2009). Furthermore, during early puberty (mean age 11.9 years), clear volumetric sex differences were found in brain areas mediating aggression (i.e., amygdala, striatum, rostral anterior cingulate cortex, and superior temporal gyrus; male volume $>$ female volume) (Peper et al. 2009). However, testosterone levels at this age could not explain these brain morphological sex differences. It might therefore be argued that a possible influence of testosterone on brain areas involved in aggression has a prenatal or early postnatal origin.

In conclusion, testosterone is unmistakably involved in human aggression and contributes importantly to sex differences in aggressive behavior. These sex differences, however, seem to originate before puberty. The relative increase of testosterone levels in adolescent boys and its relation to aggressive behavior is obscured by at least two mediators: high testosterone-sensitivity due to high prenatal testosterone levels and, especially during adolescence, levels of basal cortisol. Taking these factors into account, increased levels of testosterone enhance aggressive behavior in both adolescent boys and girls. Thus, it seems that the link between testosterone and aggression in adolescents is maintained. The heredescribed relations between prenatal, adolescent and adult levels of testosterone, together with results found after testosterone administration, are in support of Archer's hypothesis that sex differences in aggression are a result of sexual selection.

\section{Development of sex differences in physical aggression: The maternal link to epigenetic mechanisms}

\author{
doi:10.1017/S0140525X09990288
}

\section{Richard E. Tremblay ${ }^{\mathrm{a}, \mathrm{c}}$ and Sylvana M. Côté $\mathrm{e}^{\mathrm{b}, \mathrm{c}}$ \\ ${ }^{a}$ School of Public Health and Population Sciences, University College Dublin; Belfield Campus, Dublin 4, Ireland; ${ }^{\mathrm{b}}$ Department of Social and Preventive Medicine and St-Justine Hospital, University of Montreal, Montreal, Quebec H3T 1J7, Canada; ' International Laboratory for Children's Mental Health, University of Montreal, Montreal, Quebec H3T 1J7, Canada and INSERM, Paris, France.}

tremblar@grip.umontreal.ca sylvana.cote@umontreal.ca http://www.gripinfo.ca/Grip/Public/www/

\begin{abstract}
As Archer argues, recent developmental data on human physical aggression support the sexual selection hypothesis. However, sex differences are largely due to males on a chronic trajectory of aggression. Maternal characteristics of these males suggest that, in societies with low levels of physical violence, females with a history of behavior problems largely contribute to maintenance of physical aggression sex differences.
\end{abstract}

We agree with Archer that sex differences in physical aggression during infancy observed in recent longitudinal studies support the sexual selection theory rather than the social role/biosocial theory. We first emphasize that the physical aggression developmental trajectory trends observed in these studies are still more strongly at odds with the "social role/biosocial" and "social learning" theories of physical aggression. Second, we suggest that in societies with low levels of physical violence females may play a 
more active role in maintaining physical aggression sex differences than generally concluded from sexual selection theory.

Trends in physical aggression development. Results from large longitudinal studies on the development of anger and physical aggression during early childhood became available only recently. These studies clearly confirmed Darwin's (1872/1989) observations that humans use physical aggression to fight for property as soon as they have the required motor control to push, slap, hit, and kick (Alink et al. 2006; Tremblay et al. 1999).

As illustrated in our Figure 1, analyses of physical aggression developmental trajectories during early childhood showed that frequency of aggression increases dramatically for most children from its onset at the end of the first year after birth until it reaches a peak between the third and fourth year (Côté et al. 2007). If this general increase is driven by social learning of aggression (Bandura 1973), it is unlikely to be from imitation of virtual violent models. Still, it could be learned from parents, siblings, and peers. Yet, reliance on social learning theory of aggression poses major challenges to account for life-span sex differences. The main challenge comes from the observation, as illustrated in Figure 1, that the peak frequency of physical aggressions between 3 and 4 years is followed by a steadily decreasing trend for most children until adulthood (Côté et al. 2006; Loeber et al. 2005; Nagin \& Tremblay 1999; National Institute of Child Health and Development [NICHD] 2004). Counterintuitively, the frequency of physical aggression declines during periods (middle childhood and adolescence) when exposure to physical aggression in the neighborhood and the media increases dramatically.

This observation was totally unexpected from the "social learning of aggression" and "social role/biosocial" perspectives. Why would children suddenly reverse the developmental trend presumably set by their family environment during the first three years of life? To our knowledge no "social role" or "social learning" theorist has suggested that the majority of infants learn to aggress from their parents and unlearn from the mass media and the neighborhood. The fact that boys are more physically aggressive than girls by 17 months (Alink et al. 2006; Baillargeon et al. 2007) points to the powerful role of biological factors (Dionne et al. 2003), as Archer points out. However, these biological factors can be the product of early environmental conditions as well as genetic inheritance.

Epigenetic effects of maternal care. Developmental trajectory studies from childhood to adulthood show that a small group of children maintain high frequencies of physical aggression (see Fig. 1). This exception to the rule is useful to search for the environmental and genetic differences between the majority who learn not to physically aggress from early childhood onwards and the minority that maintains an atypically high frequency of physical aggression. As argued by Archer, males are more likely to be on the high trajectory. There is also accumulating evidence that specific genetic profiles, especially for males, are involved in neurological deficits which handicap learning alternatives to physical aggression (Buckholtz \& Meyer-Lindenberg 2008). However, much of the variance in physical aggression

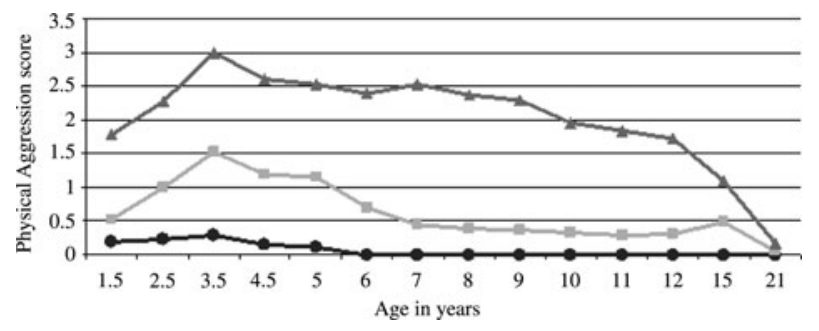

Figure 1 (Tremblay \& Côté). Developmental trajectories of physical aggression from 17 months to 21 years based on two Québec population samples: 17 months to 5 years $(\mathrm{N}=1758)$, 6 to 21 years $(\mathrm{N}=2000)$. is accounted for by environmental factors related to maternal characteristics such as the mother's history of behavior problems during adolescence; low levels of education; childbearing during adolescence; smoking during pregnancy; depression; and coercive parenting early after birth (Côté et al. 2006; Nagin \& Tremblay 1999; NICHD 2004).

Research on nonhuman primates has shown the importance of early maternal care on the development of unregulated aggression (Suomi 2005), while rodent work on early maternal behavior shows differential sex effects on play-fighting, sexual behavior, and reproductive success (Cameron et al. 2008; Parent \& Meaney 2008). There is also growing evidence from human studies of early environmental genetic programming effects which can explain differences in brain development and regulation of behavior, including aggression and suicide (McGowan et al. 2009 Tremblay 2008). From this perspective a key proximal mechanism for sex differences in aggression may be strongly based on maternal behavior during the perinatal period through its impact on infant gene expression, brain development, and behavior development, and eventually on the next generation through reproductive behavior (Gluckman \& Hanson 2005; Meaney 2001).

The link between maternal behavior and frequent male physical aggression appears important to understand the reason we observe relatively large physical aggression sex differences in modern societies despite the fact that the use of this form of aggression to resolve conflicts is largely condemned for males and females. Indeed, frequency of physical aggression is one of the best predictors of male school failure and exclusion from the labor market (Kokko \& Pulkkinen 2000; Vitaro et al. 2005). Longitudinal studies of females with physical aggression problems during childhood show that, although they do not maintain high levels of physical aggression during adolescence and early adulthood, they tend to fail in school, suffer from depression, are likely to mate with behavior problem males, to become pregnant during adolescence, to smoke during pregnancy, and to use coercive behavior towards their children (Fontaine et al. 2008; Serbin et al. 1998). In other words, they have all the risk factors needed to place their male children on a trajectory of chronic physical aggression. From this perspective males who mate with well-adapted females should in the long run contribute to the reduction of physical aggression sex differences among humans. It can also be argued that helping maladjusted females adapt to the modern environment will increase the likelihood that their male children will be less physically aggressive and thus reduce sex differences in physical aggression.

\section{Sexual selection and social roles: Two models or one?}

doi:10.1017/S0140525X09990355

\section{Pierre L. van den Berghe \\ Department of Sociology, University of Washington, Seattle, WA 98195-3340. plvdb@u.washington.edu}

Abstract: Nothing is gained by opposing "sexual selection" and "social
roles," or by proclaiming the supremacy of one over the other. Instead,
we should develop a unitary model of gene-culture coevolution,
allowing for the complex interaction of both, and varying importance of
each, all within our double, species-specific, adaptive, evolutionary track.

Archer's article is a useful survey of the evidence in support of the role of sexual selection in male-female differences in the expression of physical violence and risk-taking, combined with a weak and inconsistent conceptual framework. Archer begins by opposing two views of gender differences in the expression of aggression, "sexual selection theory" and "social role/biosocial theory," and, after reviewing the evidence, proclaims the first a hands-down winner. Then, he turns to conflicts between mates, 
notes a lesser sex difference in aggression, and shifts gears to propose an economics-style model of conflict between selfish maximizers in a dyadic relationship. Even then, he concedes that cultural variation leaves a large, unexplained residual.

All this conceptual confusion can be simply resolved by discarding the opposition between the two approaches, and adopting a unitary model of gene-culture coevolution. There is abundant evidence for the role of sexual selection in producing gender differences in behavior, including levels of aggression. But the same sexual selection model also explains sexually differentiated behavior between mates in a reproductive relationship. Indeed, that is the very core of sexual selection. No conceptual shift is needed at all. It is all one and the same.

Now for cultural variation: It, too, obviously exists, but by now it is clear that the circular explanation of culture in terms of culture is bankrupt. "They do it so because it is their custom" is not an alternative model of behavior. It merely describes; it does not explain. Cultural variation is not random; most of it is adaptive, and linked to adaptation by natural selection. Humans are on a double evolutionary track: genetic and cultural, complexly intertwined. A single coevolution model explicating variations in gene-culture interactions is called for (Alexander 1979; Boyd \& Richerson 1985; Chagnon \& Irons 1979; Lumsden \& Wilson 1981). In some instances, cultural variation is near zero. In others, it is considerable. That range of variation is the explanandum.

Let us take the example of women's participation in warfare. It ranges from negligible to considerable (Adams 1983; Lynn 2008). But a closer look reveals a near-zero variation in women's direct participation in combat, while, in highly militarized societies (such as Israel), women play a large supporting role in scores of military duties, except combat.

In short, nothing is gained by trying to show that sexual selection explains behavior better than "social role" or culture. Everything is gained by explaining the complex modalities of geneculture coevolution in terms of a double adaptive track. To deny the importance of either is untenable.

\section{Author’s Response}

\section{Refining the sexual selection explanation within an ethological framework}

doi:10.1017/S0140525X09990963

\author{
John Archer \\ School of Psychology, University of Central Lancashire, Preston, \\ Lancashire PR1 2HE, United Kingdom. \\ jarcher@uclan.ac.uk \\ http://www.uclan.ac.uk/scitech/research/rae2008/psychology/ \\ staff_profiles/jarcher.php
}

\begin{abstract}
My response is organized into three sections. The first revisits the theme of the target article, the explanatory power of sexual selection versus social role theory. The second considers the range and scope of sexual selection, and its application to human sex differences. Two topics are examined in more detail: (1) the paternity uncertainty theory of partner violence; (2) evolution of inter-group aggression. Section 4 covers ultimate and proximal explanations and their integration within an ethological approach. I consider the development of sex differences in aggression, and their causal mechanisms, within this framework.
\end{abstract}

\section{R1. Introduction}

I thank the commentators for an interesting and varied set of comments on my target article. Most of my responses are organized in terms of the following broad themes. The first is the central question in my target article, whether sex differences in aggression are better explained by sexual selection than social role theory. Most commentators agree that sexual selection is preferable, although a minority do not view the two as incompatible. The second issue concerns sexual selection. A number of commentators raise the issue of how broadly or narrowly sexual selection should be defined, and what other features of human sex differences might be covered by the concept. Some want me to go further than I had in expanding its scope, and offer their own varied and interesting suggestions as to how this could be achieved. Several of these are clearly advances on aspects of my target article, whereas others (in my opinion) are not. Arising out of this discussion of sexual selection are two issues that require longer treatment: violence between partners and inter-group aggression. The third main theme concerns ultimate and proximal explanations of human behavior. My preferred approach to evolutionary psychology is grounded in ethology, rather than the modular version. I outline the main differences between these two and then consider commentaries relating to development and causation within the ethological framework.

\section{R2. Sexual selection and social role theory}

Most commentators agree that sexual selection provides a more comprehensive explanation of sex differences in aggression than the social role theory alternative. Some (Buss; Figueredo, Gladden, \& Brumbach [Figueredo et al.]; Gaulin; Kenrick \& Griskevicius; and Pound, Daly, \& Wilson [Pound et al.]) suggest that social role theory has very little merit in this context, and that the case for sexual selection is much stronger than I indicated. Only Eagly \& Wood defend social role theory fully. Two other commentaries (from Bailey, Oxford, \& Geary [Bailey et al.] and van den Berghe) regard the explanations as reconcilable, although neither explains how this might be achieved.

Bailey et al. state: "We argue that Archer's review, along with many previous contributions to this debate, assume, either implicitly or explicitly, that sexual selection and social learning are alternative explanations - but in fact, they are not necessarily so" (first para.; emphasis theirs). Subsequently, they refer to "social roles" rather than "social learning." Yet I was careful to distinguish the older, more limited, social learning view from social role theory, and its updated biosocial version. Bailey et al. did not recognize these distinctions. They correctly state that social learning and sexual selection theories are not necessarily alternatives, since sexual selection is an ultimate explanation and social learning is one form of proximal mechanism. However, the appropriate alternative to sexual selection is the "biosocial" version of social role theory, which sets out a way in which sex differences could have arisen without sexual selection (or at least without a central role for it).

Referring to their "biosocial" social role theory, Eagly \& Wood state that "flexibility in behavior is at the heart of 
our evolutionary analysis." There are two issues raised by this statement: The first concerns flexibility, and the second the extent to which their analysis is evolutionary. The capacity for flexibility is an issue on which evolutionary psychologists and social role theorists can agree in principle, although their conceptualization is different. Gangestad et al. (2006a) put forward an evolutionarybased theory of the impact of ecology on culture (and consequently flexibility in behavior), which they explicitly contrasted with Eagly's social role theory (see also Kenrick \& Griskevicius). This approach has been further developed by others (Fincher et al. 2008; Schaller \& Murray 2008; Thornhill et al. 2009). In the target article, I considered flexibility in relation to environmental conditions (sect. 2.8). Pound et al. developed this further in their commentary, with the prediction that cues to greater fitness variance in the local environment should result in more dangerous confrontations between men. They note some of the ways in which this argument could have been further developed in my target article, in particular important recent evidence linking inequality of resource distribution and violence proneness. To add to their suggestions is the comprehensive analysis by Wilkinson and Pickett (2009), linking societal inequality with not only violent crime, but also a range of indicators of health and well-being. This reasoning could also be linked with criminological evidence on the role of marriage, work, and fatherhood in lowering the prevalence of criminal offending (Laub et al. 1998; Skarðhamar \& Lyngstad 2009).

The second issue Eagly \& Wood's statement raises is that few evolutionary researchers would recognize their analysis as "evolutionary," since they invoke evolution of the body but not the mind (as noted by Friedman et al. 2000; Lieberman 2006; and the commentaries by Browne and Kenrick \& Griskevicius). Where Wood and Eagly (2002) invoked evolved capacities, they specified that these are "physical and reproductive" (p. 719), not psychological. This view of evolution is a crucial difference between an approach rooted in sexual selection and Wood and Eagly's biosocial theory. Social role theory was originally a theory that (unlike evolution) was not concerned with ultimate origins (Eagly 1987). Later, following critiques by evolutionary psychologists (see target article), Wood and Eagly (2002) developed a version of social role theory that did include ultimate origins, one that involved constraints on what men and women could easily do in the light of their evolved physical differences. This recasting of social role theory seems to me to have been a fundamental error. Rather than seek to revise a theory with flawed assumptions (that men's and women's evolved psyches are similar), a more realistic strategy would be to acknowledge that there was an evolved origin for certain aspects of human sex differences, and to seek to incorporate social roles within that framework.

As in a previous book chapter (Eagly et al. 2004), Eagly \& Wood interpret findings on the hormonal responses to social events, such as insults, competition, and forthcoming fatherhood, as representing role demands. However, these specific reactions occur widely in other mammals, in birds and fish (Archer 1988; 2006b; see R.4), and even in cockroaches (Kou et al. 2008). It would seem more parsimonious to conceptualize them as part of a widespread pattern of adaptive responses, which have an ancient phylogenetic history, rather than as role demands that are presumably specific to humans.

As Gaulin notes, sexual selection is a theory that applies to all sexual species, and it is special pleading to argue that a different sort of theory can apply to humans, even with additions from the biological sciences, such as hormonal reactions. Indeed, this seems to do nothing but highlight fundamental problems with the theory. Sell's commentary provides a lucid analysis of the requirements for adaptive features, or for learned byproducts. He assesses Wood and Eagly's biosocial theory according to the second category, pointing out that it involves evolved sex differences in size and strength combined with a mechanism incorporating general cost-benefit analysis. He then asks why natural selection would have produced sex differences in size and strength if men and women were aggressing at similar rates. This is a telling question that is not addressed by social role theory.

\section{R3. Extending the concept and scope of sexual selection}

In this section, I consider a number of related issues, connected to the scope of sexual selection. The first subsection concerns the concept itself, its original scope and more recent extensions. This leads to a more detailed consideration of a specific evolutionary explanation that applies to post-copulatory competition: mate guarding arising from paternity uncertainty. I then consider suggestions for extending the original scope of sexual selection to other forms of behavior, and consider in more detail intergroup aggression.

\section{R3.1. The concept of sexual selection}

After Trivers' (1972) consideration of parental investment, it became logical to extend what we consider as sexual selection to processes following fertilization, although noting that this changes the original conception. I did so in the target article (sect. 4.6), and this was the theme of a recent article by Carranza (2009), a source cited in two commentaries (Campbell and Pellegrini). Specifically, I considered the application of the game theory model of Clutton-Brock and Parker (1995) to physical aggression between partners: again it should be stressed that models such as this one are quite different from classic sexual selection models (a point not appreciated by van den Berghe). Two commentaries (Cashdan and Kaighobadi \& Shackelford) were concerned that I did not consider mate guarding derived from paternity uncertainty, the usual evolutionary explanation applied to partner violence. This is also a model that involves processes following fertilization. My reasons for omitting it in the target article are elaborated in section R3.2.

Of the two original processes involved in sexual selection, I focused on male competition because it was most relevant to the topic of my article, sex differences in aggression. While recognizing that female choice was the other process, I did not cover this for reasons of relevance. However, one issue concerning female choice is raised in two commentaries (Behme and Kempenaers \& Forstmeier). It concerns the conflict between choosing aggressive or non-aggressive mates, and forms part of the extensively investigated topic of when women prefer 
men who are likely to show more parental effort and when they prefer men who typically show mating effort, and with it higher physical attraction and more pronounced aggression. These alternative male reproductive strategies were mentioned in my target article (sect. 2.7), but not in relation to women's preferences, which is another (extensive) topic. A full treatment of the evolution of sex differences in aggression would include this.

A number of commentaries seek to extend my central argument, based on inter-male competition, in interesting and relevant ways. These include intrasexual competition by females, which has been the subject of recent articles on sexual selection in animals (considered by Campbell and Pellegrini). One of these, Clutton-Brock (2009), described instances where females compete for breeding territories and other resources necessary for reproduction, and for social rank: In some cases females can show a greater variance in reproductive rate than males. These and other examples of female competition are readily encompassed by traditional conceptions of sexual selection in terms of parental investment or reproductive rate (Trivers 1972; Clutton-Brock \& Vincent 1991). They typically occur in polyandrous and monogamous species, or in eusocial breeders. Clutton-Brock (2009) also pointed out a fundamental distinction between competition in the two sexes, that male competition is typically for access to the other sex and female competition is typically for resources necessary for reproduction. This is likely to provide an additional reason why female aggression is usually escalated to a lesser extent than is male aggression.

As Clutton-Brock (2004, p. 26) noted: "one of the problems in writing about sexual selection today is that the term is used in so many different ways." Campbell's consideration of her evolutionary explanation of less risky female aggression shows the difficulty of drawing the line in specific instances. I wonder whether it really is useful when considering sex differences in behavior to extend the concept of sexual selection to such an extent that, "We might say that, under this definition, almost all selection is sexual selection" (Carranza 2009, p. 750).

Since I was mainly concerned in the target article with inter-male competition, a central part of the original conception of sexual selection (Darwin 1871/1901), it was clear what I was dealing with, and what I was not. Campbell makes a compelling case for considering other features that might not have arisen from inter-male competition, and yet which are relevant to sex differences in aggression. Behme suggests that larger male size and musculature may be fitness-enhancing outside the realms of reproduction: Of course, this may or may not be the case, but unlike Campbell's example of greater female fear, no precise functions are suggested. Until they are, it is more parsimonious to regard the enhanced upper body musculature of the human male as part of a range of adaptive features that originated from intermale competition.

Even those who propose a widening of the term sexual selection (e.g., Carranza 2009, cited by Campbell and Pellegrini) recognize the central importance of competition for mates in the process. Secondary differences, such as in feeding behavior or habitat use (Carranza 2009), may be relevant to sex differences associated with men's specialization for hunting and women's for gathering, although this arose much later in human evolution and, unlike male competition, is restricted to humans, rather than being widespread in many animals. It may well play a part in men's adaptations for group-cooperation and out-group aggression (Browne and Johnson \& van Vugt).

In his cross-species analysis of sexual selection, CluttonBrock (2009) stated that sex differences in behavior are probably more flexible than is commonly realized. This highlights the importance of ecological conditions for variations in the sex differences. Pellegrini refers to the degree to which sex differences in behavior are moderated by different ecologies. Related to this, in section 3.7, I described a cross-cultural test of the hypothesis that sex differences in size would be more pronounced in polygynous societies (Alexander et al. 1979). The original claim that this was the case was undermined by later re-analyses. A related issue concerns the relationship between mating systems and the ecological conditions under which they occur. I also mentioned the finding that polygny was associated with higher degrees of pathogen stress across 186 societies (Low 1990). Low (1989) found that boys were taught to strive and compete more in polygynous societies. Thus, the degree of pathogen stress was related to the mating system, which in turn was related to the type of socialization. More recent studies have extended Low's analysis to characteristics of the social structure of modern states, including gender empowerment (Thornhill et al. 2009). This is a particularly interesting line of research suggesting that social roles can be explained in terms of ecological adaptations.

\section{R3.2. Partner violence and paternity uncertainty}

One particular evolutionary model involving post-copulatory conflicts of interest between the sexes has been used to explain male violence to women. This is viewed as a form of mate guarding, whose ultimate function is to ensure paternity. Since females do not have the adaptive problem of paternity uncertainty, they do not have this reason to mate-guard. This explanation is elaborated in two commentaries (Cashdan and Kaighobadi \& Shackelford).

The logic of mate-guarding and its widespread existence in nonhuman animals (e.g., Parker 1974b; Wilson \& Daly 1992) is not disputed. It is likely to play a part in explaining men's sexual coercion to their partners (as Kaighobadi \& Shackelford argue), which might also be linked to sperm competition (widely found in insects and birds). However, I know of only one study that provides direct evidence for sperm competition in humans (Baker \& Bellis 1993), and as far as I know this has not been replicated.

I did not refer to the mate-guarding view as an explanation of sex differences in partner violence in my target article, although I was aware of work by Wilson and Daly $(1992 ; 1996)$ and others. I like the logic of mateguarding, and the associated emotion (sexual jealousy) is clearly associated both with men's violence to other men and with men's violence to their own partners (Daly \& Wilson 1988; 1990). It was in hindsight an omission not to have mentioned paternity uncertainty in relation to inter-male aggression motivated by sexual jealousy, since this would be in addition to the pre-copulatory intermale competition typically covered by sexual selection. The reason I did not mention paternity uncertainty in 
relation to partner violence was not an oversight. It was because there are typically no sex differences in overall acts of physical aggression, at least in modern Western nations (Archer 2000a; 2002). There are sex differences in injuries, the consequences of these acts - a difference better explained by the size and strength differences of the two sexes than by mate-guarding.

Faced with the evidence of similar proportions of male and female physical aggression to partners, it would seem reasonable to conclude that whatever part mate-guarding plays in the male psyche, this alone cannot explain partner violence. It would then be of interest to examine not only behavior used by males to increase their chances of paternity, but also behavior used by females to maximize their partner's investment in the relationship. Buss's studies of mate-retention tactics (Buss 1988a; Buss \& Shackelford 1997a) and Flinn's (1988) study of mateguarding in a Caribbean village did adopt such a "gender-inclusive" approach," although others have only studied male-to-female violence (e.g., Buss et al. 2008; Shackelford et al. 2005a). A digression is necessary on the background to such selective studies that only consider partner violence as a one-way process.

Selective studies are based on the assumption that most partner violence is male-to-female. I presented a considerable body of evidence from Western nations that this is incorrect (both in the target article and in previous reviews). The sources cited by Cashdan were ideologically biased, as are many in this area. Her source of criticism of the Conflict Tactics Scale (CTS), the measure used by family researchers to study conflict between partners, is a selective narrative review that is more a polemic than an objective review (Dobash et al. 1992). A more representative, quantitative assessment of the evidence for the accuracy and reliability of the CTS (Archer 1999) showed that it is much more reliable than Dobash et al. inferred from their review. Correlations between couples were $r=.55$ and .53 , for men and women, respectively, for an aggregate of six studies. This is comparable with values obtained for spousal ratings of personality (McCrae \& Costa 1990) and does not take into account attenuation by measurement error. In their study of 350 couples in New Zealand, Moffit et al. (1997) found that correlations of $r=.58$ and .54 were raised to .83 and .71 when latent correlations were derived from confirmatory factor analysis. Other evidence supports both the reliability and validity of the CTS and its modifications (Straus 1990; 2004), as does the similarity in the findings from this measure and other methods, such as crime victimization surveys (e.g., Laroche 2005; Mirrlees-Black et al. 1998).

Cashdan also cites the influential but controversial work of Michael Johnson (see Dutton 2005; Dutton \& Nicholls 2005). Again, I was aware of this, but chose not to cite it in the target article. Johnson (1995) argued from a qualitative review of studies involving general population samples and samples of women victims from shelters, that there were two "types" of partner violence, one (characteristically male) involving high levels of sustained violence and control, found in shelter samples, and the other mutual lower-level violence in the absence of overall control, found in general samples. He termed these, respectively, "patriarchal" (later "intimate") terrorism," and "common (later "situational") couple" violence.
Johnson (2001) applied cluster analysis to two samples, a community sample and one selected for high levels of male-to-female violence, finding clusters corresponding to these samples. It is likely that these arose from the prior selection of the samples, which in turn corresponded to Johnson's earlier classification.

Johnson's other empirical study (Johnson \& Leone 2005) used a sample drawn from a "violence against women" survey that cannot be regarded as an unbiased sample of violence by both sexes (Archer 2000a; 2002; Straus 1999). In an analysis of a large-scale Canadian survey sampling physical aggression and controlling behavior by both sexes, LaRoche (2005) found that $38 \%$ of those who fitted Johnson's "intimate terrorist" category (i.e., they were both violent and controlling) were women, again suggesting that the previous categorization was based on biased sampling. Using another sample not selected for high levels of victimization, and including reports by both men and women, Graham-Kevan and Archer (submitted) found that the "intimate terrorist" group contained similar proportions of men and women. Nonviolent victims - that is, those who do not use any physical aggression towards an "intimate terrorist" - were twice as likely to be men than women. These findings indicate that Johnson's typology should be viewed with some skepticism, rather than the uncritical acceptance it enjoys at present, and that it requires further investigation.

To return to paternity uncertainty, if this were a full explanation of partner violence, the following would be the case: (1) men but not women would show physical aggression to their partners; (2) the male-only nature of physical aggression would be consistent across cultures (since paternity uncertainty is universal); (3) males but not females would show controlling behavior to their partners; (4) male aggression but not female aggression (if and when it occurs) would be motivated by the desire to control the partner's behavior; (5) males would show more sexual jealousy than females; (6) physical aggression to a partner would be linked to sexual jealousy in males but not in females (again if and when it occurs).

The first of these expectations was mentioned above and was covered in the target article. Studies in Western nations show similar frequencies of acts of physical aggression among women and men, although women are more likely to be injured. The second was also covered in the target article, and as indicated, there is considerable and meaningful variability across nations, which is related to women's degree of societal power and to gender attitudes (Archer 2006a). The third is refuted by a number of studies showing no sex differences in controlling behavior (Felson \& Outlaw 2007; Graham-Kevan \& Archer, in press; Walby \& Allen 2004), or more control by women than men (Charles \& Perreira 2007), all from large or representative samples. The fourth is refuted by evidence that control motives are linked to physical aggression in both sexes (Felson \& Outlaw 2007; Graham-Kevan \& Archer, in press). Regarding sex differences in sexual jealousy, some studies have found none (Bookwala et al. 1992; Felson 2002; Mullen \& Martin 1994; White 1981), whereas others have found that women report more sexual jealousy than men (de Weerth \& Kalma 1993; Felson \& Outlaw 2007; Nannini \& Meyers 2000; Webb 2007). Physical aggression has been found to be related to sexual jealousy in both sexes in some studies (Felson 
1997; 2002; Haden \& Hojjat 2006), or in men only (Archer \& Webb 2006; Webb 2007), or in women more than in men (de Weerth \& Kalma 1993).

Altogether, the above findings are not what one would expect if male mate-guarding, derived from paternity uncertainty, were responsible for partner violence. However much I agree that the paternity uncertainty hypothesis makes evolutionary sense, the evidence from modern Western nations indicates that women are also seeking to control men. Having said this, I accept the view that paternity uncertainty is likely to be more important in relation to sexual aggression to partners (Kaighobadi \& Shackelford), and that men's mate-guarding is likely to show subtle changes in relation to the woman's fertility (as indicated by Sefcek \& Sacco). I also acknowledge a different point made by Johnson \& van Vugt, about male aggression towards women, that it is underestimated if men's aggression to out-group women, during warfare (and in other contexts), is ignored.

\section{R3.3. Extending the range of human behavior covered by sexual selection}

Several commentaries seek to extend the range of human behavior that could be explained by sexual selection, without necessarily extending the concept itself. These included artistic and other displays (Sefcek \& Sacco), the behavior of homosexual males (Dickins \& Sergeant), and the large sex difference in "conduct disorders" (Dickins \& Sergeant) - that is, antisocial disorders in children and adolescents, which are two and a half times more frequent in males than in females. All of these seem appropriate extensions of the scope of sexual selection in its original meaning.

Schredl's interesting and unusual commentary extends the scope of sexual selection to the content of dreams. He reports that dreams reflect real-life sex differences in not only sexual activity but also physical aggression. These findings parallel research on homicidal fantasies: Men report more of these than women do, and their fantasies are more frequent and persistent (Crabb 2000; Kenrick \& Sheets 1993). Schredl also notes that the sex differences in dream content have been stable since the 1940s and were unchanged during the time when societal gender roles changed. Consistent with this, Kenrick \& Griskevicius also note that homicide rates do not differ with changes in gender attitudes from 1960s onwards. The sex difference in physical aggression in dreams becomes pronounced from around 17 years of age, consistent with analyses of violent crimes.

Several commentators suggest that my sexual selection analysis could have been further refined by paying attention to the particular forms of aggression that sexual selection would predict. Buss does so in relation to specific adaptive problems (associated with his modular framework: see sect. R4). Kempenaers \& Forstmeier set out a number of specific predictions about male aggression derived from sexual selection. These provide useful hypotheses for future studies.

Corr \& Perkins raised the issue of whether the sexes differ in different forms of aggression, distinguishing between competitive and provoked (and also predatory) aggression. There have been many suggested subtypes of aggression, and these are discussed elsewhere (Gendreau
\& Archer 2005). The more commonly used distinction between proactive and reactive aggression (Vitaro \& Brendgen 2005) is likely to correspond to Corr \& Perkins' competitive and provoked aggression. It would therefore be interesting to enquire whether men and women differ more in either proactive or reactive aggression. These forms are typically not distinguished in most of the available studies involving sex differences, although many of the items on the commonly used physical aggression scale of the Aggression Questionnaire (A.H. Buss \& Perry 1992) do seem to refer to reactive forms of aggression. Studies specifying reactive and proactive aggression in adolescents and adults (Archer \& Thanzami, in press; Raine et al. 2006) have involved only males. It is likely that men show more of both of these forms of aggression, and that it is its physical nature, rather than the presence or absence of an immediate provocation, that is important for the sex difference. Proactive and reactive aggression are often highly correlated, indicating that they co-occur in the same individuals (e.g., Brown et al. 1996; Dodge \& Coie 1987; Raine et al. 2006), although they are related to different variables (Raine et al. 2006; Scarpa \& Haden 2006).

\section{R3.4. Beyond sexual selection: Inter-group competition}

One suggested extension of sexual selection that requires longer consideration is inter-group conflict. The principles of sexual selection apply to a range of species, and are therefore not specifically designed for the human case where inter-group competition is widespread. I mentioned inter-group competition as a limitation to the existing psychological and criminological evidence on male aggression. Recent research (e.g., van Vugt et al. 2007; Wilson \& Wrangham 2003; Wrangham 1999; Wrangham \& Wilson 2004) has considered coalitional aggression from an evolutionary viewpoint. Several commentators (e.g., Johnson \& van Vugt) raise this issue as a limitation to a sexual selection view of sex differences in aggression that is based on aggression between individuals. How to integrate evolutionary approaches to inter-group and intra-group aggression is an important issue.

Benenson argues that the contrasting structures of male and female social groups lead to a more complex consideration of sexual selection than that proposed in the target article. In particular, she characterizes males as oriented primarily towards inter-group competition, with mechanisms for minimizing within-group aggression; female competition is characterized as being aimed at removing rivals. This is a very interesting and elegant extension of my target article, but it has the drawback of being inconsistent with evidence on adult friendship patterns.

Benenson's argument involves the contrasting nature of male and female groups, which has been noted for some time in the developmental literature (e.g., Archer 1992a; Maccoby 1988; see also commentaries by Johnson \& van Vugt and Pellegrini). Benenson extends this to claim that "human males invest more than females in, and exhibit more tolerance towards, same-sex peers." She goes on to characterize males' same-sex relationships as being interconnected or groupbased (in contrast to those of females, which are not). Benenson's citations in support her position rely heavily on 
child samples. Research on adult friendship patterns suggests a different female pattern to one characterized by the lack of a group basis, and fragile friendships. In many ways, both sexes have similarly patterned friendship networks, with certain reliable differences. Men's friendships tend to be centered on shared activities whereas women's involve shared feelings. In contrast to Benenson's statement that "Human females' same-sex dyadic relationships endure for shorter periods than those of males," women's dyadic pairs are found to be more intimate, disclosing, and satisfying than those of men (e.g., Caldwell \& Peplau 1982; Dindia \& Allen 1992; Rubin 1985; Wright 1988).

The social exclusion Benenson mentions refers to the outcome of female aggression in young children. It is, however, advantageous for both sexes to exclude competitors, and in the nonhuman world social exclusion results from direct aggression. In rodents, it is typically the fate of those who are displaced following territorial disputes (Archer 1970). Disruptive young male rhesus monkeys may be driven from their social group before puberty by adult females (Suomi 2005). Throughout human history, social exclusion from a group has been used as a form of punishment (e.g., Ruff 2001). Exclusion is therefore a consequence of aggression, and not connected to one particular form, indirect (or relational) aggression, as Benenson indicates. I considered this type of aggression throughout my target article. It works better in the denser and more elaborate social groups found among women and girls (Green et al. 1996; Lagerspetz et al. 1988), than in the dyadic context that Benenson suggests is typical of female networks; here a different form of relational manipulation is found (Archer \& Coyne 2005). In terms of immediate costs, such as physical retaliation, indirect aggression is less risky than direct aggression (Björkqvist 1994), but it can indeed be effective in removing a competitor. Recent evidence suggests that women's indirect aggression is targeted particularly at those viewed as posing a reproductive threat - that is, more overtly attractive women (Vaillancourt \& Sharma 2008) - which is consistent with other analyses, including the perceptive evolutionary account of indirect aggression by Figueredo et al. To sum up, although Benenson's commentary opens up an interesting avenue for future discussion and research, it conflicts with the present evidence on men's and women's social networks.

Rather than seeking to characterize men's conflicts as inter-group and women's as within-group, it is more realistic to regard men as engaging in both inter- and intragroup conflicts, as Johnson \& van Vugt do. These commentators suggest that I have underestimated the extent of sex differences in aggression by concentrating on within-group aggression. I did acknowledge inter-group aggression in passing, and I referred to some of the sources they cite, including warfare being the common cause of male deaths in pre-state societies. There still remains a considerable amount of human aggression that is within-group. It is this that is the major topic of psychological and criminological investigations, and can most readily be linked to sexual selection.

Nevertheless, an additional section on inter-group conflict would undoubtedly have strengthened and extended the scope of my article. The magnitude of the sex difference is clearly much larger for inter-group aggression.
Male inter-group aggression is not readily explained in terms of classic sexual selection principles, but is likely to be based on sexually selected features (as Johnson \& van Vugt suggest). Male sexually selected characteristics, such as upper body strength and willingness to escalate encounters to dangerous levels, form the basis of simple forms of inter-group violent contests. It is but a short step from the coalitional aggression found in nonhuman primates to the simpler forms of group violence characteristic of humans. Van Vugt et al. (2007) identified a further psychological adaptation for such inter-group conflicts in men: Men are more likely than women to cooperate in the face of inter-group competition (see also target article). On the face of it, this would tend to decrease intra-group male competition. Yet, higher levels of male than female aggression are nevertheless reported in individual-level studies. It has to be noted, however, that studies on sex differences in inter-group aggression and conflict are only just beginning, and their data base is as yet very small compared to that covering within-group aggression, and it largely consists of laboratory studies. There are other studies (see Kenrick \& Griskevicius) indicating that activating mating motives can lead men to conform less to group norms. The full picture regarding intra- and inter-group conflict awaits clarification by future research.

Browne's commentary also concerns coalitional aggression, although not specifically about the nature of men's and women's groups. He argues that the sex differences outlined in the target article would prevent women from being as effective or as suited to combat as men. To be convincing, Browne's argument needs to include the sort of "male warrior" adaptations discussed by Johnson \& van Vugt. One finding that may be relevant in this context is the early sex difference in preferences for games and entertainments involving killing (Benenson et al. 2008).

There is a counterargument to Browne's position. Both physical and psychological sex differences - even those with large effect sizes such as upper body strength - overlap, so that there will always be some women who possess these characteristics more than some men do. As stated, Browne's argument seems to neglect such individual variability, and the importance of motivation, general physical training, and specific combat training. Consequently, it may well be that most men are more fitted for combat than most women, but this still leaves the few exceptions. It is likely that throughout history gender attitudes have converted these overlapping differences into categorical ones by generalizing from the typical sex difference to one that is prescribed by social rules. The argument that women can form effective combat units is usually illustrated by the example of the fighting units of the West African Dahomey kingdom in the eighteenth and nineteenth centuries (as noted by Eagly \& Wood). It is also likely that women have fought effectively alongside men, particularly in twentieth-century liberation armies. The position that women lack psychological adaptations for inter-group conflict could simply mean that it is more difficult, but not impossible, to integrate women as effectively as men into combat roles. How the infrequent cases discussed by Eagly \& Wood, and more extensively by Goldstein (2001), have overcome such adaptations provides an interesting challenge for future evolutionary researchers in this field. 


\section{R4. A framework for integrating ultimate and proximal explanations}

Although Buss and I agree in terms of the general position that sex differences in human aggression can be understood in terms of sexual selection, I welcome the opportunity to set out ways in which our general approaches differ. Implicit in my coverage of the relationship between evolutionary (ultimate) and immediate causal (proximal) explanations was an ethological framework. I shall now make this more explicit, in order to address the remaining commentaries. Based on Huxley's "three major problems of biology" (to which he added a fourth), Tinbergen (1963) set out four questions that need to be addressed (and integrated) when we ask why behavior occurs.

\section{R4.1. Tinbergen's four questions}

The first question, regarding "survival value," corresponds to the functional evolutionary explanations found in evolutionary psychology. As set out in Buss's commentary and elsewhere (e.g., Buss 2004; Buss \& Duntley 2006; Buss \& Shackelford 1997b), Buss's approach is to list a number of survival and reproductive problems, and to argue that there are specific mental modules designed to solve these problems. Tinbergen (1963) described three other types of explanation in addition to survival value (or function). One was evolution, or phylogenetic history, which is typically applied to the evolution of display characteristics in nonhumans (see Archer 1992b, pp. 149-75), and in humans, to nonverbal signals (e.g., Andrew 1963; van Hooff 1972). The evolutionary background of the mechanisms underlying aggression, and how they might differ in the two sexes, is particularly important for distinguishing an ethologically based evolutionary approach from the modular approach, and also for addressing issues raised in other commentaries.

From an ethological viewpoint, the modular approach seems to have omitted consideration of the process of natural selection. New adaptive mechanisms are built into already-evolved structures, rather than being designed as specific mental modules for new adaptive problems. The neuroendocrine mechanisms underlying aggression and aggressive displays, and the effector organs used in fighting, illustrate this process. Humans still possess the same basic emotions derived from a phylogenetically ancient fight-flight system: more recentlyevolved neural circuitry has been incorporated into older systems rather than forming new and separate structures.

Development and causation are the other types of explanation described by Tinbergen (1963), and considerations of the process of evolutionary change imply that the links between these and function are not necessarily straightforward. Any particular adaptive function may be fulfilled by one of several possible mechanisms, and may develop in different ways. Many examples can be found in studies of adaptive behavior in animals, such as kin recognition and mate attraction. The only requirement of the developmental and causal mechanisms underlying adaptive behaviour is that they produce a particular end-product regularly in the environment in which that behaviour evolved.

Thus, causal mechanisms are not necessarily mapped on to specific adaptive problems (as in the modular view), although this does occur in some cases. Perhaps the mechanism closest to this is the ethological concept of the fixedaction pattern, whereby a specific stimulus evokes a specific response (e.g., Lorenz 1971). Mechanisms underlying aggression may range from this sort, as in the case of pain-induced aggression (Archer 1988; 1989-90), through emotional reactions, which can be seen as filling "the gap between fixed action patterns and impeccable rationality" (Johnson-Laird \& Oatley 1992, p. 206), to more specific decision-processes, responsive to local cost-benefit contingencies. The mechanisms underlying human aggression are a complex mixture of these processes, as evidenced by theories that emphasize emotions (Archer 1976; 1988; Baumeister et al. 1996; Berkowitz 1962 2008; Dollard et al. 1939), and those stressing rational goal-directed behavior (Tedeschi \& Felson 1994). Aggressive behavior has a very old phylogenetic history, so that more recently evolved mechanisms are built upon, and arise out of, older systems, rather than being created anew for each particular adaptive problem.

\section{R4.2. Development}

From the previous section, it should be clear that I do not agree that "evolutionary theory predicts the early emergence of sex differences in direct aggression" (Cashdan). Rather, in the target article I stated: "the course of development cannot be precisely specified from an evolutionary explanation" (sect. 2.1.2). Nevertheless, certain possibilities can be ruled out: for example, evolved sex differences in aggression could not be totally reliant on culture.

Eagly \& Wood comment that I attributed to them the view that "sex difference should start small and increase with age through childhood, coincident with the cumulative influence of socialization." This was a statement taken from Table 1, summarizing predictions derived from sexual selection and social role theories. In the text, it was elaborated, where I stated that this was a reasonable inference from social learning accounts of sex differences in aggression, which emphasize the cumulative influence of socialization through childhood. Eagly \& Wood note that social role accounts have "remained silent" on the issue of age trends in aggression. Yet social role (and "biosocial") accounts incorporate social learning as one of the mechanisms underlying the transmission of social roles, thus making it reasonable to infer that they are in agreement with the processes described in social learning accounts, in particular the gradual learning over time of aggressive behavior from role models.

Tremblay \& Côté make the same point about social learning in their commentary, noting that "Counterintuitively, the frequency of physical aggression declines during periods (middle childhood and adolescence) when exposure to physical aggression in the neighborhood and the media increases dramatically." As they put it, no social learning or social role theorist has suggested that infants learn to aggress from their parents and subsequently unlearn this when they become exposed to influences from outside the family and from the media. From this perspective, it is difficult to understand why Behme thinks that "it is difficult to see how a theory of sexual selection can explain that the amount of aggressive behaviour decreases steadily 
throughout childhood." She goes on to argue that "we should expect the highest values of aggression [...] at the time when mating occurs." There is no requirement for the various traits associated with inter-male competition to arise at the same time during development, only that they are all in place by the time of maximum inter-male competition. Behme's comment again assumes that functional origins and development are necessarily closely linked. It also conflates the assessment of aggression based on frequency of acts (which is greatest at 2-3 years of age) with their damaging nature (which peaks in young adulthood). It is the effectiveness of physical aggression that is likely to result in success in reproductive-related competition, not how often feeble motor acts occur. As noted by Tremblay et al. (1999), and in subsequent work (e.g., Côté et al. 2006), the decline in physical aggression with age during childhood comes about through the learning of alternative responses to physical aggression, a process that differs among individuals and between the sexes, and is influenced by a range of maternal characteristics (Tremblay \& Côté).

Pellegrini poses the question of why there is no early sexual dimorphism in size and strength when there is early dimorphism in behavior. His interesting suggestion is that sex differences in physical activity and locomotor play are developmental precursors for the size and strength differences that result from the actions of testosterone at puberty.

Several authors highlight the interactive nature of the development of sex differences in aggression, and note the differential environmental influences on males and females. Tremblay \& Côté emphasize the importance of maternal influences, particularly a number of high risk factors such as early childrearing and smoking during pregnancy - these predict high levels of physical aggression by males. They comment that even though such mothers do not themselves show the high levels of physical aggression more characteristic of males, "they have all the risk factors needed to place their male children on a trajectory of chronic physical aggression." Their sons will fit the pattern of showing a reproductive strategy characterized by a bias towards mating rather than parental effort (sect. 2.7 in the target article). In a similar vein, the work of Boden and his colleagues showed that an early adverse family environment was more predictive of partner violence for men than for women. Men showed less continuity in their aggression than women did (which, Boden notes, would not be expected from a social role view).

\section{R4.3. Causation}

Tinbergen's remaining question, in addition to function, evolution, and development, concerns causation, the immediate internal and external influences on behavior. Some commentators Cashdan, Finkel \& Slotter, and Pound et al.) questioned the inference that there should not be a sex difference in anger, the emotion that underlies aggression, although others (e.g., Campbell) accepted it. The reason given in the target article is that both sexes have reasons for becoming angry but the costs and benefits of the behavior resulting from the anger are different. This would fit the finding (discussed below) that the sex difference in physical aggression is mediated by risky impulsivity. It would also be consistent with Daly and Wilson's (1988, pp. 163-86) emphasis on risky strategies underlying the evolved greater male propensity to violent conflicts. Daly and Wilson stated at the beginning of this chapter: "Men become embroiled in dangerous competitive interactions far more often than do women" (p. 163). I suggested that it is the interactions resulting from anger that are more dangerous in men than in women.

Nevertheless, it is possible to see how the motivational mechanism behind angry aggression could have been changed so that men and women differed in the extent to which they become angry. In their "culture of honor" studies, Nisbett and Cohen (1996) found that it was the angry response to certain provoking situations that differentiated people from an honor culture, and those who were not. If men and women differed in the same way, this would produce a sex difference in anger to honorrelated situations, even though general measures of anger might not reveal such a difference. In this respect, the final paragraph of the commentary by Pound et al. is the most pertinent. If it is the state of becoming angry that entails the elevated risk, we should predict a sex difference in the ease of becoming angry in men and women, but only in certain circumstances (e.g., competitive exchanges with members of the same sex). On the other hand, when men perceive physical aggression to have high costs, it is likely that they still experience anger: it is the behavior that is different from when the costs are lower. Applying this to women, the calibration of the level for high costs could simply be set lower. Thus, women's behavior (but not their initial emotional reaction) would be more inhibited by the costs of physical aggression, as Campbell (1999) suggested. There are pros and cons to both sides of this argument, and while I found the commentary by Pound et al. almost persuasive, I had lingering doubts. Their suggestion provides several interesting avenues for future research.

As indicated in section R4.1, causation is a separate issue to function, and in my view it needs to be addressed in a different way than listing adaptive problems and assuming modules in the mind corresponding to each one (Buss). In line with this, I considered possible mediators of the sex difference in aggression, but noted that there were currently no empirical tests of these. Campbell's commentary describes such a test. In devising a measure of risk, she was careful to include only risks that would have potentially dangerous consequences, as these had been identified as the relevant forms in evolutionary analyses (e.g., Campbell 1999; Daly \& Wilson 1988). Controlling for this measure of risk-taking did eliminate the usual sex differences in physical and verbal aggression.

Finkel \& Slotter suggested that my argument concerning the mediation of sex differences in aggression could be extended with reference to " $\mathrm{I}^{3}$ Theory." This is an analysis of impelling and inhibiting motivational forces. It involves a binary classification of variables that should be included in any motivational model of aggressive behavior. My article concerned those variables that mediated sex differences in aggression, and therefore would not take into account all the inhibiting and impelling forces controlling aggression. I have two observations on $\mathrm{I}^{3}$ Theory. The first is that it seems to be a recasting of a cost-benefit analysis, 
which was discussed in the target article, and has been applied to the study of several forms of aggression (Archer et al., submitted; Archer \& Southall 2009; Rutter \& Hine 2005). The second is that it appears not to be a theory in the sense that specific hypotheses can be generated, but a list of variables that make aggression more or less likely. This may be useful for organizing our thinking of the various influences on forms of aggression, such as partner aggression (Finkel 2007), but it would seem to add little or nothing to how the motivational system underlying aggression is organized. For example, Finkel \& Slotter's penultimate paragraph merely recasts what I (and others) have written about the variability in partner violence - that different balances in the societal power of men and women alter the immediate costbenefit calculus for resorting to physical violence against a partner.

In the target article, I stated that the rising levels of testosterone in male adolescents were not associated with an increase in male aggression, as occurs in other species. Elsewhere (Archer 2006b), I argued that the behaviorhormone relationships in humans fit a pattern identified previously by Wingfield et al. (1990) in birds, known as "the challenge hypothesis." According to this, testosterone rises at puberty, but to moderate levels that support growth and reproductive functioning and behavior, but it does not directly facilitate aggression. Both sexual arousal and challenges involving young males do lead to short-term rises in testosterone levels, and these facilitate competitive behavior, including aggression. Terburg, Peper, Morgan, \& van Honk (Terburg et al.) argue that behavioral studies indicate that testosterone is a mediator of aggression; however, the studies they cite are correlational, and there is abundant evidence that aggressive behavior can lead to increased testosterone levels (Archer 2006b; Kemper 1990). Terburg et al.'s commentary goes on to make the important point that interactions between testosterone and cortisol - found in several studies - have been neglected in such analyses: The influence of testosterone appears to be stronger in individuals with low cortisol levels, although in some cases the two hormones increase together (Cohen et al. 1996).

Terburg et al. also mention findings of a positive association between aggression and digit ratio (2D:4D), regarded as a marker for prenatal testosterone levels. From this, they suggest that prenatal testosterone may affect the sensitivity to later circulating testosterone (as it does in the classic studies of the organizing effects of perinatal testosterone). Thus, Terburg et al. draw our attention to two specific and testable variables (cortisol and prenatal testosterone) that might moderate the association between testosterone and aggression from puberty onwards.

\section{R5. Conclusions}

Future research is likely to arise from a widening of the concept and scope of sexual selection. A number of commentaries referred to theoretical extensions of sexual selection theory beyond the two processes originally described by Darwin (1859/1911; 1871/1901). These involved consideration of a number of selection pressures, which may lie outside the classic definition of sexual selection, but which are nonetheless important for a full account of sex differences in aggression. Some of these were referred to in the target article, others were not. Foremost is the theory by Campbell (1999), that women avoid costly encounters to increase their survival chances for rearing offspring. Added to this is the consideration that women do compete for mates, but in less damaging ways than men do. Inter-group conflicts were only mentioned in passing in the target article, but these are clearly of great importance when considering aggression by human males. Adaptations for group conflict are likely to have been built on to those already in place for intra-group conflict.

Modern extensions of sexual selection theory extend to post-copulatory competition, notably that between males and females. This was covered in the target article in connection with a game-theory model of the evolution of coercion in animals (sect. 5). I did not include the paternity uncertainty theory of male partner violence, and in my response explained that this was because the evidence from industrialized Western nations on physical aggression between partners did not seem to support its predictions. It nevertheless remains important for considering inter-male aggression during the post-copulatory phase (omitted from the target article), and for considering sexual aggression and subtle forms of mate guarding that are likely to become coercive where restraints on males' behavior are low.

Section R4 provided an opportunity to outline the different approaches taken by evolutionary psychologists grounded in ethological theory and those adopting a modular framework. The ethological approach involves four interconnected explanations of behavior, rather than functions and their mental representations, as in the modular approach. I then used the ethological framework to consider a number of issues concerning development and causation. In particular, learning to decrease physical aggression at an age when there are abundant role models for aggression was discussed in relation to the classic social learning account, which would not seem to predict this. The question of the causal mediating influences for sex differences in aggression raised the issue of why the sexes apparently do not differ in anger. My position in the target article was that the difference resides in how men and women respond to anger, but following a commentary, here in the response I discussed the possibility that there could be differences in reactions to provocation. This is just one example of a number of avenues for future research opened up by the commentaries on my article, and I thank all the commentators for their very interesting and diverse comments.

\section{References}

[The letters " $a$ " and “ $r$ " before author's initials stand for target article and response references, respectively.]

Adams, D. (1983) Why there are so few women warriors. Behavior Science Research 18:196-212. [PLvdB]

Addison, W. E. (1989) Beardedness as a factor in perceived masculinity. Perceptual and Motor Skills 68:921-22. [aJA]

Alcock, J. (1998) Animal behavior, 6th edition. Sinauer Associates. [AS] 
Alexander, R. D. (1979) Darwinism and human affairs. University of Washington Press. [PLvdB]

(1987) The biology of moral systems. Hawthorne. [DDPJ]

Alexander, R. D., Hoogland, J. L., Howard, R. D., Noonan, K. M. \& Sherman, P. W. (1979) Sexual dimorphisms and breeding systems in pinnipeds, ungulates, primates, and humans. In: Evolutionary biology and human social behavior, ed. N. A. Chagnon \& W. Irons, pp. 402-35. Duxbury Press. [arJA]

Alink, L. R. A., Mesman, J., van Zeijl, J., Stolk, M. N., Juffer, F., Koot, H. M., Bakermans-Kranenburg, M. J. \& van IJzendoorn, M. H. (2006) The early childhood aggression curve: Development of physical aggression in 10- to 50month-old children. Child Development 77(4):954-66. [CB, RET]

Alpern, S. B. (1998) Amazons of Black Sparta: The women warriors of Dahomey. New York University Press. [AHE]

American Psychiatric Association (2000) Diagnostic and Statistical Manual of Mental Disorders, Fourth Edition, Text Revision. American Psychiatric Association. [DSM-IV Diagnostic Criteria 312.8 accessed online on 12 March 2009 from: http://www.behavenet.com/capsules/disorders/cndctd.htm.] [TED]

Andersson, M. (1994) Sexual selection. Princeton University Press. [aJA, CB, SJCG]

Andrew, R. J. (1963) The origin and evolution of calls and facial expressions of the primates. Behaviour 20:1-109. [rJA]

Apicella, C. L., Feinberg, D. R. \& Marlowe, F. W. (2007) Voice pitch predicts reproductive success in male hunter gatherers. Biological Letters 3:682-84. [aJA]

Archer, J. (1970) Effects of population density on behaviour in rodents. In: Social behaviour in birds and mammals, ed. J. H. Crook, pp. 169-210. Academic Press. [rJA]

(1976) The organisation of aggression and fear in vertebrates. In: Perspectives in ethology 2, ed. P. P. G. Bateson \& P. Klopfer, pp. 231-98. Plenum. [rJA]

(1988) The behavioural biology of aggression. Cambridge University Press. [arJA]

(1989-90) Pain-induced aggression: An ethological perspective. Current Psychology: Research and Reviews 8:298-306. [rJA]

(1992a) Childhood gender roles: Social context and organisation. In: Childhood social development: Contemporary perspectives, ed. H. McGurk, pp. 31-61. Erlbaum. [arJA]

(1992b) Ethology and human development. Barnes \& Noble. [rJA]

(1994) Violence between men. In: Male violence, ed. J. Archer, pp. 121-40. Routledge. [aJA]

(1996) Sex differences in social behavior: Are the social role and evolutionary explanations compatible? American Psychologist 51:909-17. [aJA]

(1999) Assessment of the reliability of the Conflict Tactics Scales: A meta-analytic review. Journal of Interpersonal Violence 14:1263-89. [arJA]

(2000a) Sex differences in aggression between heterosexual partners: A meta-analytic review. Psychological Bulletin 126:651-80. [arJA, PJC, AHE] (2000b) Sex differences in partner aggression: A reply to Frieze (2000), O'Leary (2000) and White, Smith, Koss, and Figueredo (2000). Psychological Bulletin 126:697-702. [aJA]

(2002) Sex differences in physically aggressive acts between heterosexual partners: A meta-analytic review. Aggression and Violent Behavior: A Review Journal 7:313-51. [arJA]

(2004) Sex differences in aggression in real-world settings: A meta-analytic review. Review of General Psychology 8:291-322. [aJA]

(2006a) Cross-cultural differences in physical aggression between partners: A social-role analysis. Personality and Social Psychology Review 10:113-33. [arJA]

(2006b) Testosterone and human aggression: An evaluation of the challenge hypothesis. Neuroscience and Biobehavioral Reviews 30:319-45. [arJA, AHE]

(submitted) Derivation and assessment of a hypermasculine values questionnaire. [aJA]

Archer, J. \& Côté, S. (2005) Sex differences in aggressive behavior: A developmental and evolutionary perspective. In: Developmental origins of aggression, ed. R. Tremblay, W. W. Hartup \& J. Archer, pp. 425-43. Guilford Press. [aJA]

Archer, J. \& Coyne, S. M. (2005) An integrated review of indirect, relational, and social aggression. Personality and Social Psychology Review 8:212-30. [arJA]

Archer, J., Fernández-Fuertes, A. \& Thanzami, V. L. (submitted) Assessing whether a cost-benefit analysis or self-control predicts involvement in two forms of aggression. $\quad[\mathrm{rJA}]$

Archer, J. \& Lloyd, B. B. (2002) Sex and gender, 2nd edition. Cambridge University Press. [aJA]

Archer, J. \& Mehdikhani, M. (2003) Variability among males in sexually-selected attributes. Review of General Psychology 7:219-36. [aJA]

Archer, J. \& Southall, N. (2009) Does cost-benefit analysis or self-control predict involvement in bullying behavior by male prisoners? Aggressive Behavior 35:31-40. [rJA]
Archer, J. \& Thanzami, V. L. (2007) The relation between physical aggression, size and strength, among a sample of young Indian men. Personality and Individual Differences 43:627-33. [aJA]

(in press) The relation between mate value, entitlement, physical aggression, size, and strength, among a sample of young Indian men. Evolution and Human Behavior. DOI: 10.1016/jevolhumbehav.2009.03.003. [r]A]

Archer, J. \& Webb, I. A. (2006) The relation between scores on the Buss-Perry Aggression Questionnaire and aggressive acts, impulsiveness, competitiveness, dominance, and sexual jealousy. Aggressive Behavior 32:464-73. [rJA]

Arnold, D. (1997) Co-occurrence of externalizing behavior problems and emergent academic difficulties in young high-risk boys: A preliminary evaluation of patterns and mechanisms. Journal of Applied Developmental Psychology $18: 317-30$. [CB]

Bailey, A. A. \& Hurd, P. L. (2005) Finger length ratio (2D:4D) correlates with physical aggression in men but not women. Biological Psychology 68:215-22. [TED, DT]

Bailey, R. O., Seymour, N. R. \& Stewart, G. R. (1978) Rape behavior in blue-winged teal. Auk 95:188-90. [FK]

Baillargeon, R. H., Zoccolillo, M., Keenan, K., Côté, S., Pérusse, D., Wu, H.-X., Boivin, M. \& Tremblay, R. E. (2007) Gender differences in physical aggression: A prospective population-based survey of children before and after 2 years of age. Developmental Psychology 43(1):1326. [aJA, RET]

Baker, R. R. \& Bellis, M. A. (1993) Human sperm competition: Ejaculate adjustment by males and the function of masturbation. Animal Behaviour 46:861-85. [rJA, FK]

(1995). Human sperm competition. Chapman \& Hall. [JAS]

Bales, R. F. \& Borgatta, E. F. (1955) Size of group as a factor in the interaction profile. In: Small groups: Studies in social interaction, ed. A. P. Hare, E. F. Borgatta \& R. F. Bales, pp. 495-512. Random House. [JFB]

Bandura, A. (1973) Aggression: A social learning analysis. Holt/ Prentice-Hall. [aJA, RET]

Barash, D. P. (1977) Sociobiology of rape in mallards (Anas platyrhynchos): Response of the mated male. Science 197:788-89. [FK]

Bateman, A. J. (1948) Intrasexual selection in Drosophila. Heredity 2:349-68. [aJA]

Bateson, P. P. G. \& Martin, P. (1999) Design for a life: How behaviour develops Jonathan Cape. [ADP]

Battié, M. C., Bigos, S. J., Fisher, L. D., Hansson, T. H., Jones, M. E. \& Wortley, M. D. (1989) Isometric lifting strength as a predictor of industrial back pain reports. Spine 14:851-56. [aJA]

Baumeister, R. F., Smart, L. \& Boden, J. M. (1996) Relation of threatened egotism to violence and aggression: The dark side of high self-esteem. Psychological Review 103:5-33. [r]A]

Becker, D. V., Kenrick, D. T., Neuberg, S. L., Blackwell, K. C. \& Smith, D. M. (2007) The confounded nature of angry men and happy women. Journal of Personality and Social Psychology 92:179-190. [aJA]

Becker, S. W. \& Eagly, A. H. (2004) The heroism of women and men. American Psychologist 59:163-78. [KRB]

Belsky, J., Steinberg, L. \& Draper, P. (1991) Childhood experience, interpersonal development, and reproductive strategy: An evolutionary theory of socialization. Child Development 62:647-70. [TED]

Benenson, J. F., Antonellis, T. J., Cotton, B. J., Noddin, K. E. \& Campbell, K. A. (2008a) Sex differences in children's use of coalitions to obtain scarce resources. Animal Behaviour 76:497-505. [JFB]

Benenson, J. F., Apostoleris, N. H. \& Parnass, J. (1997) Age and sex differences in dyadic and group interaction. Developmental Psychology 33:538-43. [JFB]

Benenson, J. F., Carder, H. P. \& Geib-Cole, S. J. (2008) The development of boys' preferential pleasure in physical aggression. Aggressive Behavior 34:154-66. [arJA]

Benenson, J. F. \& Christakos, A. (2003) The greater fragility of females'versus males' closest same-sex friendships. Child Development 74:1123-29. [JFB]

Benenson, J. F., Maiese, R., Dolenszky, E., Dolensky, N., Sinclair, N. \& Simpson, A. (2002) Group size regulates self-assertive versus self-deprecating responses to interpersonal competition. Child Development 73:1818-29. [JFB]

Benenson, J. F., Markovits, H., Fitzgerald, C., Geoffroy, D., Fleming, J., Kahlenberg, S. \& Wrangham, R. W. (2009) Males' greater tolerance of same-sex peers. Psychological Science 20:184-90. [JFB]

Benenson, J. F., Morganstein, T. \& Roy, R. (1998) Sex differences in children's investment in peers. Human Nature 9:369-90. [JFB]

Benenson, J. F., Nicholson, C., Waite, A., Roy, R. \& Simpson, A. (2001) The influence of group size on children's competitive behavior. Child Development 72:921-28. [JFB]

Benenson, J. F., Saelen, C., Markovits, H. \& McCabe, S. (2008b) Males derive greater benefits than females do from same-sex peers relative to parents. Evolutionary Psychology 6:13-28. [JFB]

Berenbaum, S. A. \& Hines, M. (1992) Early androgens are related to childhood sextyped toy preferences. Psychological Science 3:203-206. [DHB] 
Berenbaum, S. A. \& Resnick, S. M. (1997) Early androgen effects on aggression in children and adults with congenital adrenal hyperplasia. Psychoneuroendocrinology 22:505-15. [aJA, TED]

Bergeron, N. \& Schneider B. (2005) Explaining cross-national differences in peerdirected aggression: A quantitative synthesis. Aggressive Behavior 2:116-37. [CB]

Berkowitz, L. (1962) Aggression: A social psychological analysis. McGraw-Hill. [rJA]

(1993) Aggression: Its causes, consequences and control. McGraw-Hill. [aJA]

(2008) On the consideration of automatic as well as controlled psychological processes in aggression. Aggressive Behavior 34:117-29. [rJA]

Bernal, V. (2000) Equality to die for?: Women guerrilla fighters and Eritrea's cultural revolution. PoLAR: Political and Legal Anthropology Review 23:61-76. [AHE]

Bettencourt, B. A. \& Kernahan, C. (1997) A meta-analysis of aggression in the presence of violent cues: Effects of gender differences and aversive provocations. Aggressive Behavior 23:447-56. [aJA, PJC]

Bettencourt, B. A. \& Miller, N. (1996) Gender differences in aggression as a function of provocation: A meta-analysis. Psychological Bulletin 119:422-47. [aJA, TED]

Betzig, L. (1986) Despotism and differential reproduction: A Darwinian view of history. Aldine. [aJA]

(1992) Roman polygyny. Ethology and Sociobiology 13:309-49. [aJA]
[a]

Biernat, M. (2003) Toward a broader view of social stereotyping. American Psychologist 58:1019-27. [AHE]

Birkhead, T. R., Hunter, F. M. \& Pellatt, J. E. (1989) Sperm competition in the zebra finch, Taeniopygia guttata. Animal Behaviour 38:935-50. [FK]

Bjorklund, D. J. \& Pellegrini, A. D. (2000) Child development and evolutionary psychology. Child Development 71:1687-708. [aJA]

Björkqvist, K. (1994) Sex differences in physical, verbal and indirect aggression: A review of recent research. Sex Roles 30:177-88. [arjA]

Björkqvist, K., Österman, K. \& Lagerspetz, K. (1994) Sex differences in covert aggression among adults. Aggressive Behavior 20:27-33. [aJA]

Blanchard, D. C. (1997) Stimulus and environmental control of defensive behaviors. In: Learning, motivation, and cognition: The functional behaviorism of Robert C. Bolles, ed. M. Bouton \& M. Fanselow, pp. 283-305. American Psychological Association. [PJC]

Blanchard, D. C., Hynd, A. L., Minke, K. A., Minemoto, T. \& Blanchard, R. J. (2001) Human defensive behaviors to threat scenarios show parallels to fearand anxiety-related defense patterns of non-human mammals. Neuroscience and Biobehavioral Reviews 25:761-70. [PJC]

Blau, J. \& Blau, P. (1982) The cost of inequality. American Sociological Review 47:114-29. [NP]

Blurton Jones, N. (1972) Categories of child-child interaction. In: Ethological studies of child behaviour, ed. N. Blurton Jones, pp. 97-127. Cambridge University Press. [aJA]

Book, A. S., Starzyk, K. B. \& Quinsey, V. L. (2001) The relationship between testosterone and aggression: A meta-analysis. Aggression and Violent Behavior: A Review Journal 6:579-99. [aJA]

Bookwala, J., Frieze, I. H., Smith, C. \& Ryan, K. (1992) Predictors of dating violence: A multivariate analysis. Violence and Victims 7:297-311. [rJA]

Borgerhoff Mulder, M. (2004) Are men and women really so different? Trends in Ecology and Evolution 19:3-5. [ADP]

Boulton, M. J. (1994) The relationship between playful and aggressive fighting in children, adolescents and adults. In: Male violence, ed. J. Archer, pp. 23-41. Routledge. [aJA]

Bowles, S. (2006) Group competition, reproductive leveling, and the evolution of human altruism. Science 314:1569-72. [DDPJ]

Boyd, R. \& Richerson, P. J. (1985) Culture and the evolutionary process. University of Chicago Press. [PLvdB]

Braun, P., Wiegand, D. \& Aschenbrenner, H. (1991) The assessment of complex skills and of personality characteristics in military services. In: Handbook of military psychology, ed. R. Gal \& A. D. Mangelsdorff, pp. 37-61. Wiley. [KRB]

Brennan, L., McDonald, J. \& Shlomowitz, R. (1997) Sex differences in Indian height at home and abroad. Man in India 77:105-18. [aJA]

Broder, A. \& Hohmann, N. (2003) Variations in risk taking behavior over the menstrual cycle: An improved replication. Evolution and Human Behavior 24:391-98. [JAS]

Brown, D. E. (1991) Human universals. McGraw-Hill. [SJCG]

Brown, K., Atkins, M. S., Osborne, M. L. \& Milnamow, M. (1996) A revised teacher rating scale for reactive and proactive aggression. Journal of Abnormal Child Psychology 24:473-80. [r]A]

Browne, K. (2001) Women at war: An evolutionary perspective. Buffalo Law Review 49:51-247. [KRB]

(2007) Co-ed combat: The new evidence that women shouldn't fight the nation's wars. Sentinel/ Penguin USA. [KRB, DDPJ]

Buckholtz, J. W. \& Meyer-Lindenberg, A. (2008) MAOA and the neurogenetic architecture of human aggression. Trends in Neurosciences 31(3):120-29. [RET]
[RT
Burriss, R. P. \& Little, C. (2006) Effects of partner conception risk phase on male perception of dominance in faces. Evolution and Human Behavior 27:297-305. [JAS]

Burt, S. A., McGue, M., DeMarte, J. A., Krueger, R. F. \& Iaconon, W. G. (2006) Timing of menarche and the origins of conduct disorder. Archives of General Psychiatry 63:890-96. [TED]

Buss, A. H. \& Perry, M. (1992) The aggression questionnaire. Journal of Personality and Social Psychology 63:452-59. [rJA]

Buss, D. M. (1988a) From vigilance to violence: Tactics of mate retention in American undergraduates. Ethology and Sociobiology 9:291-317. [rJA]

(1988b) The evolution of human intrasexual competition: Tactics of mate attraction. Journal of Personality and Social Psychology 54:616-28. [DMB]

(1996) The evolutionary theory of human social strategies. In: Social psychology: Handbook of basic principles, ed. E. T. Higgins \& A. W. Kruglanski, pp. 3-38. Guilford Press. [aJA]

(1998) Sexual strategies theory: Historical origins and current status. Journal of Sex Research 35:19-31. [JAS]

(2000) The dangerous passion. Free Press. [FK]

(2004) Evolutionary psychology: The new science of the mind, 2nd edition. Pearson. [rJA]

(2005) The murderer next door: Why the mind is designed to kill. The Penguin Press. [DMB]

Buss, D. M. \& Dedden, L. (1990) Derogation of competitors. Journal of Social and Personal Relationships 7:395-422. [DMB]

Buss, D. M. \& Duntley, J. D. (2006) The evolution of aggression. In: Evolution and social psychology, ed. M. Schaller, D. T. Kenrick \& J. A. Simpson, pp. 263-86. Psychology Press. [rJA, DMB]

(2008) Adaptations for exploitation. Group Dynamics 12:53-62. [DMB]

Buss, D. M. \& Shackelford, T. K. (1997a) From vigilance to violence: Mate retention tactics in married couples. Journal of Personality and Social Psychology 72:346-61. [rJA]

(1997b) Human aggression in evolutionary psychological perspective. Clinical Psychology Review 17:605-19. [rJA, DMB]

Buss, D. M., Shackelford, T. K. \& McKibbin, W. F. (2008) The Mate Retention Inventory-Short Form (MRI-SF). Personality and Individual Differences 44:322-34. [rJA]

Bussey, K. \& Bandura, A. (1999) Social cognitive theory of gender development and differentiation. Psychological Review 106:676-713. [aJA]

Buston, P. M. \& Emlen, S. T. (2003) Cognitive processes underlying human mate choice: The relationship between self-perception and mate preference in Western society. Proceedings of the National Academy of Sciences USA 100:8805-10. [ADP]

Byrnes, J. P., Miller, D. C. \& Schafer, W. D. (1999) Gender differences in risk taking: A meta-analysis. Psychological Bulletin 125:367-83. [aJA]

Cairns, R., Xie, H. \& Leung, M. (1998) The popularity of friendship and the neglect of social networks: Toward a new balance. In: Sociometry then and now: Building on six decades of measuring children's experiences with the peer group, ed. W. M. Bukowski \& A. H. Cillessen, pp. 25-54. Jossey-Bass. [JFB]

Caldwell, M. A. \& Peplau, L. A. (1982) Sex differences in same-sex friendships. Sex Roles 8:721-32. [rJA]

Cameron, N. M., Fish, E. W. \& Meaney, M. J. (2008) Maternal influences on the sexual behavior and reproductive success of the female rat. Hormones and Behavior 54(1):178-84. [RET]

Campbell, A. (1995) A few good men: Evolutionary psychology and female adolescent aggression. Ethology and Sociobiology 16:99-123. [aJA, DMB]

(1999) Staying alive: Evolution, culture and women's intra-sexual aggression. Behavioral and Brain Sciences 22:203-52. [arJA, JFB, AC, EC, ADP] (2002) A mind of her own: The evolutionary psychology of women. Oxford University Press. [DMB, AC]

(2006) Sex differences in direct aggression: What are the psychological mediators? Aggression and Violent Behavior 11:237-64. [aJA]

Campbell, A. \& Muncer, S. (1994) Sex differences in aggression: Social representations and social roles. British Journal of Social Psychology 33:233-40. [aJA]

(in press) Can "risky" impulsivity explain sex differences in aggression? Personality and Individual Differences. [AC]

Campbell, A., Muncer, S. \& Gorman, B. (1993) Sex and social representations of aggression: A communal-agentic analysis. Aggressive Behavior 19:125-35. [aJA]

Campbell, A., Shirley, L. \& Caygill, L. (2002) Sex-typed preferences in three domains: Do two-year-olds need cognitive variables? British Journal of Psychology 93:203-17. [aJA]

Campbell, D. W. \& Eaton, W. O. (1999) Sex differences in the activity level of infants. Infant and Child Development 8:1-17. [aJA]

Campbell, J. C. (1992) Wife-battering: Cultural contexts versus Western social sciences. In: Sanctions and sanctuary: Cultural perspectives on the beating of wives, ed. D. A. Counts, J. K. Brown \& J. C. Campbell, pp. 229-49. Westview. [aJA] 
Caro, T. M. \& Bateson, P. (1986) Ontogeny and organization of alternative tactics. Animal Behaviour 34:1483-99. [ADP]

Carranza, J. (2009) Defining sexual selection as sex-dependent selection. Animal Behaviour 77:749-51. [rJA, AC, ADP]

Carré, J. M. \& McCormick, C. M. (2008) In your face: Facial metrics predict aggressive behaviour in the laboratory and in varsity and professional hockey players. Proceedings of the Royal Society of London B 275:2651-56. Available at: http://journals.royalsociety.org/content/h80173234257qq01/ [aJA]

Carver, C. S. (2004) Negative affects deriving from the behavioural approach system. Emotion 4:3-22. [PJC]

Carver, C. S. \& Harmon-Jones, E. (2009) Anger is an approach-related affect: Evidence and implications. Psychological Bulletin 135:183-204. [PJC]

Chagnon, N. A. (1979) Is reproductive success equal in egalitarian societies? In: Evolutionary biology and human social behavior: An anthropological perspective, ed. N. A. Chagnon \& W. Irons, pp. 374-401. Duxbury. [aJA]

(1983) Yanomamo: The fierce people, 3rd edition. Holt, Rinehart \& Winston. [DMB]

(1988) Life histories, blood revenge, and warfare in a tribal population. Science 239:985-92. [DMB, DDPJ]

Chagnon, N. A. \& Irons, W. (1979) Evolutionary biology and human social behavior: An anthropological perspective. Duxbury. [PLvdB]

Charles, P. \& Perreira, K. M. (2007) Intimate partner violence during pregnancy and 1-year post-partum. Journal of Family Violence 22:609-19. [rJA]

Chen, X., Chen, H., Liu, M. \& Wang, L. (2002) Noncompliance and child-rearing attitudes as predictors of aggressive behaviour: A longitudinal study in Chinese children. International Journal of Behavioral Development 26:225-33. [CB]

Cheng, K. M., Burns, J. T. \& McKinney, F. (1983) Forced copulation in captive mallards III. Sperm competition. Auk 100:302-10. [FK]

Cheuvront, S. N., Moffatt, R. J. \& DeRuisseau, K. C. (2002) Body composition and gender differences in performance. In: Nutritional assessment of athletes, ed. I. Wolinksy \& J. A. Driskell, pp. 177-200. CRC Press. [KRB]

Chisholm, J. S. (1999) Attachment and time preference: Relations between early stress and sexual behavior in a sample of American university women. Human Nature 10:51-83. [TED]

Clutton-Brock, T. H. (2004) What is sexual selection? In: Sexual selection in primates: New and comparative perspectives, ed. P. M. Kappeler \& C. P. van Schaik, pp. 22-36. Cambridge University Press. [rJA]

(2007) Sexual selection in males and females. Science 318:1882-85. [AC, JFB, TED]

(2009) Sexual selection in females. Animal Behaviour 77:3-11. [rJA, JFB, ADP]

Clutton-Brock, T. H. \& Albon, S. D. (1979) The roaring red deer and the evolution of honest advertisement. Behaviour 69:145-70. [aJA]

Clutton-Brock, T. H. \& Harvey, P. H. (1977) Primate ecology and social organization. Journal of Zoology 183:1-39. [aJA]

Clutton-Brock, T. H., Harvey, P. H. \& Rudder, B. (1977) Sexual dimorphism, socioeconomic sex ratio and body weight in primates. Nature 269:797-800. [aJA]

Clutton-Brock, T. H. \& Parker, G. A. (1995) Sexual coercion in animal societies. Animal Behaviour 49:1345-65. [arJA]

Clutton-Brock, T. H. \& Vincent, A. C. J. (1991) Sexual selection and the potential reproductive rates of males and females. Nature 351:58-60. [arJA, SJCG]

Cohen, D. B. (1973) Sex role orientation and dream recall. Journal of Abnormal Psychology 82:246-52. [MS]

Cohen, D., Nisbett, R. E., Bowdle, B. F. \& Schwarz, N. (1996) Insult, aggression, and the Southern culture of honor: An "experimental ethnology." Journal of Personality and Social Psychology 70:945-60. [rJA]

Colapinto, J. (2001) As nature made him: The boy who was raised as a girl. Harper Collins. [DHB]

Conway, M., Irannejad, S. \& Giannopoulos, C. (2005) Status-based expectancies for aggression, with regard to gender differences in aggression in social psychological research. Aggressive Behavior 31381-89. [aJA]

Cornwell, R. \& Perrett, D. (2008) Sexy sons and sexy daughters: The influence of parents' facial characteristics on offspring. Animal Behaviour 76: 1843-53. [CB]

Corr, P. J. (2002) J. A. Gray's reinforcement sensitivity theory and frustrative nonreward: A theoretical note on expectancies in reactions to rewarding stimuli. Personality and Individual Differences 32:1247-53. [PJC]

(2008) Reinforcement sensitivity theory (RST): Introduction. In: The reinforcement sensitivity theory of personality, ed. P. J. Corr, pp. 1-43. Cambridge University Press. [PJC]

Côté, S., Boivin, M., Nagin, D. S., Japel, C., Xu, Q., Zoccolillo, M., Junger, M. \& Tremblay, R. E. (2007) The role of maternal education and non-maternal care services in the prevention of children's physical aggression. Archives of General Psychiatry 64(11):1305-12. [RET]

Côté, S., Vaillancourt, T., LeBlanc, J. C., Nagin, D. S. \& Tremblay, R. E. (2006) The development of physical aggression from toddlerhood to pre-adolescence: A nation wide longitudinal study of Canadian children. Journal of Abnormal Child Psychology 34(1):71-85. [rJA, CB, RET]
Courtwright, D. T. (1996) Violent land: Single men and social disorder from the frontier to the inner city. Harvard University Press. [aJA]

Crabb, P. B. (2000) The material culture of homicidal fantasies. Aggressive Behavior 26:225-34. [rJA]

Cronin, H. (1991) The ant and the peacock: Altruism and sexual selection from Darwin to today. Cambridge University Press. [CB]

Crook, J. H. (1989) Introduction: Socioecological paradigms, evolution and history: Perspectives for the 1990s. In: Comparative socioecology: The behavioural ecology of humans and other mammals, ed. V. S. Standen \& R. A. Foley, pp. 1-36. Blackwell Scientific. [ADP]

Cummings, M., Iannotti, R. \& Zahn-Waxler, C. (1989) Aggression between peers in early childhood: Individual continuity and developmental change. Child Development 60:887-95. [CB]

Dabbs, J. M., Jr., Carr, T. S., Frady, R. L. \& Riad, J. K. (1995) Testosterone, crime, and misbehavior among 692 male prison inmates. Personality and Individual Differences 9:269-75. [DT]

Dabbs, J. M., Jr. \& Hargrove, M. F. (1997) Age, testosterone, and behavior among female prison inmates. Psychosomatic Medicine 59:477-80. [DT]

Dabbs, J. M., Jr. \& Jurkovic, G. J. (1991) Salivary testosterone and cortisol among late adolescent male offenders. Journal of Abnormal Child Psychology 19(4):469-78. [DT]

Daigle, L. E., Cullen, F. T. \& Wright, J. P. (2007) Gender differences in the predictors of juvenile delinquency: Assessing the generality-specificity debate. Youth Violence and Juvenile Justice 5(3):254-86. [JMB]

Dale, J., Dunn, P. O., Figuerola, J., Lislevand, T., Szekely, T. \& Whittingham, L. A. (2007) Sexual selection explains Rensch's rule of allometry for sexual size dimorphism. Proceedings of the Royal Society of London B 274:2971-79. [BK]

Daly, M. \& Wilson, M. (1983) Sex, evolution and behavior, 2nd edition. Willard Grant/ Wadsworth. [aJA, AS]

(1988) Homicide. Aldine de Gruyter. [arJA, DMB, AC, EC, SJCG, FK, AS] (1990) Killing the competition: Female/female and male/male homicide. Human Nature 1:81-107. [arJA]

(2001) Risk-taking, intrasexual competition, and homicide. In: Evolutionary psychology and motivation. Nebraska Symposium on Motivation, vol. 47. ed. J. A. French, A. C. Kamil \& D. W. Leger, pp. 1-36. University of Nebraska Press. [aJA, NP]

(2005) Carpe diem: Adaptation and devaluing the future. Quarterly Review of Biology 80:55-60. [NP]

Daly, M., Wilson, M. \& Vasdev, S. (2001) Income inequality and homicide rates in Canada and the United States. Canadian Journal of Criminology 43:219-36. [NP]

Daly, M., Wilson, M. \& Weghorst, J. (1982) Male sexual jealousy. Ethology and Sociobiology 3:11-27. [FK]

Darwin, C. (1859/1911) On the origin of species by means of natural selection. Murray. [arJA, PJC]

(1871/1901) The descent of man, and selection in relation to sex. Murray. Available online at: http://www.infidels.org/library/historical/charles_ darwin/descent_of_man/chapter_08.html. [arJA, CB, DHB, SJCG, ADP]

(1871/2004) The descent of man: And selection in relation to sex. Penguin Classics. (Original work published 1871.) [AC]

(1872/1989) The expression of the emotions in man and animals. Murray/ Gryphon Editions. [RET]

Davies, N. B. \& Halliday, T. M. (1978) Deep croaks and fighting assessment in toads Bufo bufo. Nature 274:683-85. [aJA]

Davies, N. B. \& Hartley, I. R. (1996) Food patchiness, territory overlap and social systems: An experiment with dunnocks (Prunella modularis). Journal of Animal Ecology 65:837-46. [aJA]

Davies, N. B. \& Lundberg, A. (1984) Food distribution and a variable mating system in the dunnock, Prunella modularis. Journal of Animal Ecology 53:895-912. [aJA]

Dawkins, R. \& Carlisle, T. R. (1976) Parental investment, mate desertion and a fallacy. Nature 262:131-33. [aJA]

de Weerth, C. \& Kalma, A. P. (1993) Female aggression as a response to sexual jealousy: A sex role reversal? Aggressive Behavior 19:265-79. [rJA]

Destro-Bisol, G., Donati, F., Coia, V., Boschi, I., Verginelli, F., Caglia, A., Tofanelli, S., Spedini, G. \& Capelli, C. (2004) Variation in female and male lineages in sub-Saharan populations: The importance of sociocultural factors. Molecular Biology and Evolution 21:1673-82. [aJA]

Diaz, A. \& Pickering, A. D. (1993) The relationship between Gray's and Eysenck's personality spaces. Personality and Individual Differences 15:297-305. [PJC]

Dickins, T. E. (2006) Evolutionary health psychology. Health Psychology Update 15:4-10. [TED]

Dickins, T. E. \& Sergeant, M. J. T. (2008) Social dominance and sexual orientation. Journal of Evolutionary Psychology 6:57-71. [TED]

Dindia, K. \& Allen, M. (1992) Sex differences in self-disclosure: A meta-analysis Psychological Bulletin 112:106-24. [rJA] 
Dionne, G., Boivin, M., Tremblay, R. E., Laplante, D. \& Pérusse, D. (2003) Physical aggression and expressive vocabulary in 19 month-old twins. Developmental Psychology 39(2):261-73. [RET]

Dobash, R. E. \& Dobash, R. P. (1977-78) Wives: The "appropriate" victims of marital violence. Victimology: An International Journal 2:426-42. [aJA] (1979) Violence against wives. Free Press. [FK]

(1980) Violence against wives: A case against the patriarchy. Open Books. [aJA]

Dobash, R. P., Dobash, R. E., Wilson, M. \& Daly, M. (1992) The myth of sexual symmetry in marital violence. Social Problems 39:71-91. [arJA, EC]

Dodge, K. A. \& Coie, J. D. (1987) Social-information processing factors in reactive and proactive aggression in children's peer groups. Journal of Personality and Social Psychology 53:1146-58. [rJA]

Dollard, J., Doob, L. W., Miller, N. E., Mowrer, O. H. \& Sears, R. R. (1939) Frustration and aggression. Yale University Press. [rJA]

Domhoff, G. W. (1996) Finding meaning in dreams: A quantitative approach. Plenum Press. [MS]

Duntley, J. D. \& Shackelford, T. K., eds. (2008) Evolutionary forensic psychology. Oxford University Press. [DMB]

Dutton, D. G. (1998) The abusive personality. Guilford Press. [FK]

(2005) On comparing apples with apples deemed nonexistent: A reply to Johnson. Journal of Child Custody 2:53-63. [rJA]

Dutton, D. G. \& Nicholls, T. L. (2005) The gender paradigm in domestic violence research and theory: Part 1 - the conflict of theory and data. Aggression and Violent Behavior 10:680-714. [rJA]

Eagly, A. H. (1987) Sex differences in social behavior: A social role interpretation. Erlbaum. [arJA, SJCG]

(1997) Sex differences in social behavior: Comparing social role theory and evolutionary psychology. American Psychologist 52(12):1380-83. [aJA, JMB, ADP]

Eagly, A. H. \& Steffen, V. J. (1984) Gender stereotypes stem from the distribution of women and men into social roles. Journal of Personality and Social Psychology 46:735-54. [aJA]

(1986) Gender and aggressive behavior: A meta-analytic review of the social psychological literature. Psychological Bulletin 100(3):309-30. [aJA, JMB, TED]

Eagly, A. H. \& Wood, W. (1999) The origins of sex differences in human behavior: Evolved dispositions versus social rules. American Psychologist 54:408-23. [aJA, DTK, NP, JAS]

(2006) Three ways that data can misinform: Inappropriate partialling, small samples, and anyway, they're not playing our song. Psychological Inquiry 17:131-37. [aJA]

Eagly, A. H., Wood, W. \& Diekman, A. B. (2000) Social role theory of sex differences and similarities: A current appraisal. In: The developmental social psychology of gender, ed. T. Eckes \& H. M. Trautner, pp. 123-74. Erlbaum. [aJA]

Eagly, A. H., Wood, W. \& Johannesen-Schmidt, M. C. (2004) Social role theory of sex differences and similarities: Implications for the partner preferences of women and men. In: The psychology of gender, 2nd edition, ed. A. H. Eagly, A. Beall \& R. S. Sternberg, pp. 269-95. Guilford Press. [arJA]

Eaton, W. O. \& Enns, L. R. (1986) Sex differences in human motor activity level. Psychological Bulletin 100:19-28. [aJA]

Eens, M. \& Pinxten, R. (2000) Sex-role reversal in vertebrates: Behavioural and endocrinological accounts. Behavioural Processes 51:135-47. [BK]

Efoghe, G. B. (1989) Are extraverts more conjugally aggressive than introverts? A study of Nigerian couples. Indian Journal of Behaviour 13:1-9. [aJA]

Eisner, M. (2003) Long-term historical trends in violent crime. Crime and Justice: A Review of Research 30:83-142. [aJA]

Ellis, L., Hershberger, S. L., Field, E. M., Wersinger, S., Pellis, S., Hetsroni, A., Geary, D., Palmer, C. T., Karadi, K. \& Hoyenga, K. B. (2008) Sex differences: Summarizing more than a century of scientific research. Psychology Press. [DDPJ]

Else-Quest, N. M., Hyde, J. S., Goldsmith, H. H. \& Van Hulle, C. A. (2006) Gender differences in temperament: A meta-analysis. Psychological Bulletin 132 33-72. [aJA]

El-Zanty, F., Hussein, E. M., Shawky, G. A., Way, A. A. \& Kishor, S. (1995) Egypt demographic and health survey 1995. Egypt, Cairo: National Population Council. [aJA]

Emery, R. E. \& Billings-Laumann, L. (1998) An overview of the nature, causes, and consequences of abusive family relationships toward differentiating maltreatment and violence. American Psychologist 53(2):121-35. [JMB]

Emlen, S. T. \& Oring, L. W. (1977) Ecology, sexual selection and the evolution of mating systems. Science 197:215-23. [aJA]

Evans, S., Neave, N. \& Wakelin, D. (2006) Relationship between vocal characteristics and body size and shape in human males: An evolutionary explanation for a deep male voice. Biological Psychiatry 72:160-63. [aJA]
Evers, C., Fischer, A. H., Rodriguez Mosquera, P. M. \& Manstead, A. S. R. (2005) Anger and social appriaisal: A "spicy" sex difference. Emotion 5:258-66. [aJA]

Eysenck, H. J. \& Eysenck, S. B. G. (1991) Eysenck Personality Scales (EPS Adult). Hodder \& Stoughton. [PJC]

Fagot, B. (1984) The consequents of problem behavior in toddler children. Journal of Abnormal Child Psychology 12:385-96. [CB]

Fagot, B. \& Hagan, R. (1985) Aggression in toddlers: Responses to the assertive acts of boys and girls. Sex Roles 12:341-52. [CB]

Felson, R. B. (1996) Big people hit little people: Sex differences in physical power and interpersonal violence. Criminology 34:433-52. [aJA]

(1997) Anger, aggression, and violence in love triangles. Violence and Victims 12:345-62. [rJA]

(2002) Violence and gender reexamined. American Psychological Association. [arJA]

Felson, R. B. \& Outlaw, M. C. (2007) The control motive and marital violence. Violence and Victims 22:387-407. [rJA]

Fergusson, D. M., Boden, J. M. \& Horwood, L. J. (2008) Developmental antecedents of interpartner violence in a New Zealand birth cohort. Journal of Family Violence 23:737-53. [JMB]

Feshbach, N. D. (1969) Sex differences in children's modes of aggressive responses toward outsiders. Merrill Palmer Quarterly 15:249-58. [aJA, JFB]

Feshbach, N. D. \& Sones, G. (1971) Sex differences in adolescent reactions toward newcomers. Developmental Psychology 4:381-86. [JFB]

Fessler, D. M. T., Pillsworth, E. G. \& Flamson, T. J. (2004) Angry men and disgusted women: An evolutionary approach to the influence of emotions on risk-taking. Organizational Behavior and Human Decision Processes 95:107-123. [KRB]

Fiebert, M. S. \& Gonzalez, D. M. (1997) College women who initiate assaults on their male partners and the reasons offered for such behavior. Psychological Reports 80:583-90. [aJA]

Figueredo, A. J., Corral-Verdugo, V., Frias-Armenta, M., Bachar, K. J., White, J., McNeill, P. L., Kirsner, B. R. \& del Pilar Castell-Ruiz, I. (2001) Blood, solidarity, status, and honor: The sexual balance of power and spousal abuse in Sonora, Mexico. Evolution and Human Behavior 22:295-328. [aJA]

Figueredo, A. J., Gladden, P. R., Andrzejczak, D. J., Jones, D. N. \& Smith-Castro, V. (in preparation) Life history strategy, executive functioning, and negative androcenrism. $\quad[\mathrm{AJF}]$

Fincher, C. L., Thornhill, R., Murray, D. R. \& Schaller, M. (2008) Pathogen prevalence predicts human cross-cultural variability in individualism/collectivism. Proceedings of the Royal Society of London B 275:1279-85. [rJA]

Fink, B., Manning, J. T., Williams, J. H. G. \& Podmore-Nappin, C. (2007) The 2nd to 4th digit ratio and developmental psychopathology in school-aged children. Personality and Individual Differences 42:369-79. [TED]

Finkel, E. J. (2007) Impelling and inhibiting forces in the penetration of intimate partner violence. Review of General Psychology 11:193-207. [rJA, EJF] (2008) Intimate partner violence perpetration: Insights from the science of selfregulation. In: Social relationships: Cognitive, affective, and motivational processes, ed. J. P. Forgas \& J. Fitness, pp. 271-88. Psychology Press. [EJF]

Fischer, A. H. \& Roseman, I. J. (2007) Beat them or ban them: The characteristics and social functions of anger and contempt. Journal of Personality and Social Psychology 93:103-15. [EJF]

Flinn, M. V. (1988) Mate guarding in a Caribbean village. Ethology and Sociobiology 9:1-28. [rJA]

Folstad, I. \& Karter, A. K. (1992) Parasites, bright males, and the immunocompetence handicap. American Naturalist 139:603-22. [SJCG]

Fontaine, N., Carbonneau, R., Barker, E. D., Vitaro, F., Hébert, M., Côté, S. M., Nagin, D. S., Zoccolillo, M. \& Tremblay, R. E. (2008) Girls' hyperactivity and physical aggression during childhood and adjustment problems in early adulthood: A 15-year longitudinal study. Archives of General Psychiatry 65:320-28. [RET]

Ford, C. S. \& Beach, F. A. (1951) Patterns of sexual behavior. Harper \& Row. [aJA]

Forsberg, A. J. L. \& Tullberg, B. S. (1995) The relationship between cumulative number of cohabiting partners and number of children for men and women in modern Sweden. Ethology and Sociobiology 16:221-32. [aJA]

Frederick, D. A. \& Haselton, M. G. (2007) Why is muscularity sexy? Tests of the fitness indicator hypothesis. Personality and Social Psychology Bulletin 33:1167-83. [BK]

Friedman, B. X., Bleske, A. L. \& Scheyd, G. J. (2000) Incompatible with evolutionary theorizing. American Psychologist 55:1059-60. [rJA]

Frieze, I. H. (1983) Investigating the causes and consequences of marital rape. Signs: Journal of Women in Culture and Society 8:532-53. [FK]

Frijda, N., Kuipers, P. \& ter Schure, E. (1989) Relations among emotion, appraisal, and emotional action readiness. Journal of Personality and Social Psychology 57:212-28. [EJF]

Fuentes, A. (1999) Re-evaluating primate monogamy. American Anthropology 100:890-907. [aJA]

Gage, A. J. \& Hutchinson, P. L. (2006) Power, control, and intimate partner sexual violence in Haiti. Archives of Sexual Behavior 35:11-24. [FK] 
Gallup, G. G., Jr., Burch, R. L., Zappieri, M. L., Parvez, R. A., Stockwell, M. L. \& Davis, J. A. (2003) The human penis as a semen displacement device. Evolution and Human Behavior 24:277-89. [FK, JAS]

Gangestad, S. W., Haselton, M. G. \& Buss, D. M. (2006a) Evolutionary foundations of cultural variation: Evoked culture and mate preferences. Psychological Inquiry 17:75-95. [arJA, DTK]

(2006b) Toward an integrative understanding of evolved and transmitted culture: The importance of specialized psychological design. Psychological Inquiry 17:138-51. [aJA]

Garn, S. \& Clark, L. (1953) The sex difference in the basal metabolic rate. Child Development 24:215-24. [AS]

Gat, A. (2006) War in human civilization. Oxford University Press. [DDPJ]

Gaulin, S. J. C. (1992) Evolution of sex differences in spatial ability. Yearbook of Physical Anthropology 35:125-51. [SJCG]

Geary, D. C. (1998) Male, female: The evolution of human sex differences. American Psychological Association. [aJA, DHB]

(2000) Evolution and proximate expression of human paternal investment. Psychological Bulletin 126:55-77. [aJA]

(2002) Sexual selection and human life history. Advances in Child Development and Behavior 30:41-101. [aJA]

Geary, D. C., Byrd-Craven, J., Hoard, M. K., Vigil, J. \& Numtee, C. (2003) Evolution and development of boys' social behavior. Developmental Review 23:444-70. [aJA, DHB]

Gendreau, P. L. \& Archer, J. (2005) Subtypes of aggression in humans and animals. In: Developmental origins of aggression, ed. R. Tremblay, W. W. Hartup \& J. Archer, pp. 25-46. Guilford Press. [rJA]

Gergen, M. (1990) Beyond the evil empire: Horseplay and aggression. Aggressive Behavior 16:381-98. [aJA]

Gilbert, D. T. (1998) Ordinary personology. In: The handbook of social psychology, vol. 2, 4th edition, ed. D. T. Gilbert, S. T. Fiske \& G. Lindzey, pp. 89-150. McGraw-Hill. [AHE]

Gladden, P. R., Sisco, M. \& Figueredo, A. J. (2008) Sexual coercion and life history strategy. Evolution and Human Behavior 29:319-26. [AJF]

Gleason, K. A., Jensen-Campbell, L. A. \& Richardson, D. S. (2004) Agreeableness as a predictor of aggression in adolescence. Aggressive Behavior 30:43-61. [aJA]

Gluckman, P. D. \& Hanson, M. (2005) The fetal matrix: Evolution, development, and disease. Cambridge University Press. [RET]

Goetz, A. T. \& Shackelford, T. K. (2006) Sexual coercion and forced in-pair copulation as sperm competition tactics in humans. Human Nature 17:265-82. [FK, JAS]

Goetz, A. T., Shackelford, T. K., Weekes-Shackelford, V. A., Euler, H. A., Hoier, S. Schmitt, D. P. \& LaMunyon, C. W. (2005) Mate retention, semen displacement, and human sperm competition: A preliminary investigation of tactics to prevent and correct female infidelity. Personality and Individual Differences 38:749-63. [FK]

Goldstein, J. S. (2001) War and gender. Cambridge University Press. [rJA]

Goodwin, D. (1955) Some observations on the reproductive behavior of rooks. British Birds 48:97-107. [FK]

Graham-Kevan, N. \& Archer J. (in press) Control tactics and partner violence in heterosexual relationships. Evolution and Human Behavior [rJA]

(submitted) Using Johnson's domestic violence typology to classify men and women: Victim and perpetrator reports. [rJA]

Grauer, A. L. \& Stuart-Macadam, P., eds. (1998) Sex and gender in paleopathological perspective. Cambridge University Press. [DMB]

Gray J. A. \& McNaughton, N. (2000) The neuropsychology of anxiety: An enquiry into the functions of the septohippocampal system, 2nd edition. Oxford University Press. [PJC]

Gray, J. P. \& Wolfe, L. D. (1980) Height and sexual dimorphism of stature among human societies. American Journal of Physical Anthropology 53:441-56. [aJA]

Green, L. R., Richardson, D. R. \& Lago, T. (1996) How do friendship, indirect, and direct aggression relate? Aggressive Behavior 22:81-86. [rJA]

Griskevicius, V., Cialdini, R. B. \& Kenrick, D. T. (2006a) Peacocks, Picasso, and parental investment: The effects of romantic motives on creativity. Journal of Personality and Social Psychology 91:63-76. [DTK]

Griskevicius, V., Goldstein, N., Mortensen, C., Cialdini, R. B. \& Kenrick, D. T. (2006b) Going along versus going alone: When fundamental motives facilitate strategic (non)conformity. Journal of Personality and Social Psychology 91:281-94. [DTK]

Griskevicius, V., Tybur, J. M., Gangestad, S. W., Perea, E. F., Shapiro, J. R. \& Kenrick, D. T. (2009) Aggress to impress: Hostility as an evolved contextdependent strategy. Journal of Personality and Social Psychology 96:980-994. [DTK]

Griskevicius, V., Tybur, J. M., Sundie, J. M., Cialdini, R. B., Miller, G. F. \& Kenrick, D. T. (2007) Blatant benevolence and conspicuous consumption: When romantic motives elicit strategic costly signals. Journal of Personality and Social Psychology 93:85-102. [DTK]
Gross, M. R. (1996) Alternative reproductive strategies and tactics: Diversity within sexes. Trends in Ecology and Evolution 11:92-98. [aJA]

Guilaine, J. \& Zammit, J. (2004) The origins of war: Violence in prehistory. Blackwell. [DDPJ]

Guimond, S., Chatard, A., Branscombe, N. R., Brunot, S., Buunk, A. P., Conway, M. A., Crisp, R. J., Dambrun, M., Désert, M., Garcia, D. M., Haque, S., Leyens, J.-P., Lorenzi-Cioldi, F., Martinot, D., Redersdorff, S. \& Yzerbyt, V. (2007) Culture, gender, and the self: Variations and impact of social comparison processes. Journal of Personality and Social Psychology 92:1118-34. [AHE]

Gunnar, M. R., Wewerka, S., Frenn, K., Long, J. D. \& Griggs, C. (2009) Developmental changes in hypothalamus-pituitary-adrenal activity over the transition to adolescence: Normative changes and associations with puberty. Developmental Psychopathology 21(1):69-85. [DT]

Guthrie, R. D. (1970) Evolution of human threat display organs. Evolutionary Biology 4:257-302. [aJA]

Haden, S. C. \& Hojjat, M. (2006) Aggressive responses to betrayal: Type of relationship, victim's sex, and nature of aggression. Journal of Social and Personal Relationships 23:101-16. [rJA]

Hall, C. S. \& Domhoff, B. J. (1963) Aggression in dreams. International Journal of Social Psychiatry 9:259-67. [MS]

Hall, C. S., Domhoff, B. J., Blick, K. A. \& Weesner, K. E. (1982) The dreams of college men and women in 1959 and 1980: A comparison of dream contents and sex differences. Sleep 5:188-94. [MS]

Hall, C. S. \& Van de Castle, R. L. (1966) The content analysis of dreams. AppletonCentury-Crofts. [MS]

Hall, R. L. (1978) Sexual dimorphism for size in seven nineteenth-century Northwest coast populations. Human Biology 50:159-71. [aJA]

Hamel, J. \& Nicholls, T. L., eds. (2007) Family interventions in domestic violence: A handbook of gender-inclusive theory and treatment. Springer. [aJA]

Hamilton, W. D. \& Zuk, M. (1982) Heritable true fitness and bright birds: A role for parasites. Science 218:384-87. [aJA]

Harris, C. R., Jenkins, M. \& Glaser, D. (2006) Gender differences in risk assessment: Why do women take fewer risks than men? Judgment and Decision Making 1:48-63. [aJA]

Harris, M. B. (1992) Sex and ethnic differences in past aggressive behaviors. Journal of Family Violence 7:85-102. [aJA]

Hart, C. W. M. \& Pillig, A. R. (1960) The Tiwi of North Australia. Holt, Rinehart \& Winston. [DTK]

Hartup, W. (1974) Aggression in childhood: Developmental perspectives. American Psychologist 29:336-41. [CB]

(2005) The development of aggression: Where do we stand? In: Developmental origins of aggression, ed. W. Tremblay, W. Hartup \& J. Archer, pp. 3-22. Guilford. [CB]

Haselton, M. G. \& Gangestad, W. (2006) Conditional expression of women's desires and men's mate guarding across the ovulatory cycle. Hormones and Behavior 49:509-18. [JAS]

Hay, D. F., Castle, J. \& Davies, L. (2000) Toddlers' use of force against familiar peers: A precursor of serious aggression? Child Development 71:457-67. [aJA]

Hermans, E. J., Putman, P., Baas, J. M., Koppeschaar, H. P. \& van Honk, J. (2006a) A single administration of testosterone reduces fear potentiated startle in humans. Biological Psychiatry 59:872-74. [aJA]

Hermans, E. J., Putman, P. \& van Honk, J. (2006b) Testosterone administration reduces empathic behavior: A facial mimicry study. Psychoneuroendocrinology 31:859-66. [aJA, TED]

Hermans, E. J., Ramsey, N. F. \& van Honk, J. (2008) Exogenous testosterone enhances responsiveness to social threat in the neural circuitry of social aggression in humans. Biological Psychiatry 63:263-70. [aJA, DT]

Hess, N. H. \& Hagen, E. H. (2006) Sex differences in indirect aggression: Psychological evidence from young adults. Evolution and Human Behavior 27:2:31-45. [aJA]

Hewlett, B. S. (1988) Sexual selection and parental investment among Aka pygmies. In: Human reproductive behavior: A Darwinian perspective, ed. L. Betzig, M. Borgerhoff Mulder \& P. Turke, pp. 263-76. Cambridge University Press. [aJA]

Hilton, Z., Harris, G. T. \& Rice, M. E. (2000) The functions of aggression by male teenagers. Journal of Personality and Social Psychology 79:988-94. [aJA]

Hinde, R. A. \& Stevenson-Hinde, J., eds. (1973) Constraints on learning: Limitations and predispositions. Academic Press. [ADP]

Hinshaw, S. (1992) Externalizing behavior problems and academic underachievement in childhood and adolescence: Causal relationships and underlying mechanisms. Psychological Bulletin 111:127-55. [CB]

Hiraiwa-Hasegawa, M. (2005) Homicide by men in Japan, and its relationship to age, resources and risk taking. Evolution and Human Behavior 26:332-43. [aJA]

Holmberg, M. (1980) The development of social interchange patterns from 12 to 42 months. Child Development 52:448-56. [CB]

Hosken, D. \& Snook, R. (2005) How important is sexual conflict? American Naturalist 165:S1-4. [aJA] 
Howell, N. (1979) Demography of the Dobe !Kung. Academic Press. [aJA]

Howells, N. L. \& Rosenbaum, A. (2008) Examination of sex differences and type of violence exposure in a mediation model of family violence. Journal of Emotional Abuse 8(1):123-38. [JMB]

Hrdy, S. B. (1999) Mother Nature: Natural selection and the female of the species. Chatto and Windus. $\quad[\mathrm{AC}]$

(2005) Comes the child before man: How cooperative breeding and prolonged postweaning dependence shaped human potential. In: Hunter-gatherer childhoods: Evolutionary, developmental, and cultural perspectives, ed. B. S. Hewlett \& M. E. Lamb, pp. 65-91. Aldine Transaction. [ADP]

Hubbard, R. (1990) The politics of women's biology. Rutgers University Press. [CB]

Hudson, V. M. \& Den Boer, A. (2002) A surplus of men, a deficit of peace: Security and sex ratio in Asia's largest states. International Security 26:5-38. [aJA]

(2004) Bare branches: Security implications of Asia's surplus male population. MIT Press. [ajA]

Hunter, J. (1780) Account of an extraordinary pheasant. Philosophical Transactions of the Royal Society of London 70:527-35. [ADP]

Huntingford, F. A. \& Turner, A. K. (1987) Animal conflict. Chapman \& Hall. [AS]

Hyde, J. S. (1984) How large are gender differences in aggression? A developmental meta-analysis. Developmental Psychology 20:722-36. [aJA]

(1986) Gender differences in aggression. In: The psychology of gender: Advances through meta-analysis, ed. J. S. Hyde \& M. C. Linn, pp. 51-66. Johns Hopkins University Press. [aJA]

(2005) The gender similarities hypothesis. American Psychologist 60:581-92. [DHB, ADP]

Irons, W. (1979) Cultural and biological success. In: Natural selection and social behavior, ed. N. A. Chagnon \& W. Irons, pp. 257-72. Duxbury. [DHB]

Jenni, D. A. (1974) Evolution of polyandry in birds. American Zoologist 14:129-44. [aJA]

Johnson, B. T. (1989) Software for the meta-analytic review of research literatures. Erlbaum. [aJA]

Johnson, D. D. P., McDermott, R., Barrett, E., Cowden, J., Wrangham, R., McIntyre, M. \& Rosen, S. (2006) Overconfidence in wargames: Experimental evidence on expectations, aggression, gender and testosterone. Proceedings of the Royal Society of London B 273(1600):2513-20. [DDPJ]

Johnson, M. P. (1995) Patriarchal terrorism and common couple violence: Two forms of violence against women. Journal of Marriage and the Family 57:283-94. [rJA]

(2001) Conflict and control: Images of symmetry and asymmetry in domestic violence. In: Couples in conflict, ed. A. Booth, A. C. Crouter \& M. Clements, pp. 95-104. Erlbaum. [rJA]

(2006) Conflict and control: Gender symmetry and asymmetry in domestic violence. Violence Against Women 12:1003-18. [EC]

Johnson, M. P. \& Leone, J. M. (2005) The differential effects of intimate terrorism and common/situational couple violence: Findings from the National Violence Against Women Survey. Journal of Family Issues 26:322-49. [rJA, EC]

Johnson-Laird, P. N. \& Oatley, K. (1992) Basic emotions, rationality, and folk theory. Cognition and Emotion 6:201-23. [rJA]

Johnstone, R. (1995) Sexual selection, honest advertisement and the handicap principle: Reviewing the evidence. Biological Reviews 70:1-65. [CB]

Kaighobadi, F., Starratt, V. G., Shackelford, T. K. \& Popp, D. (2008) Male mate retention mediates the relationship between female sexual infidelity and female-directed violence. Personality and Individual Differences 44:142231. $[\mathrm{FK}]$

Kanazawa, S. (2000) Scientific discoveries as cultural displays: A further test of Miller's courtship model. Evolution and human behavior 21(5):317-21. [JAS]

Kavanagh, E. (2006) Debating sexual selection and mating strategies. Science 312:689-97. [AC]

Keeley, L. H. (1996) War before civilization: The myth of the peaceful savage. Oxford University Press. [aJA, DDPJ]

Keenan, K. \& Shaw, D. (1997) Developmental and social influences on young girls early problem behavior. Psychological Bulletin 121:95-113. [aJA]

Kemper, T. (1990) Social structure and testosterone: Elaborations of the socio-biosocial chain. Rutgers University Press. [rJA]

Kenrick, D. T., Gabrielidis, C., Keefe, R. C. \& Cornelius, J. (1996) Adolescents' age preferences for dating partners: Support for an evolutionary model of lifehistory strategies. Child Development 67:1499-511. [DTK]

Kenrick, D. T. \& Keefe, R. C. (1992) Age preferences in mates reflect sex differences in mating strategies. Behavioral and Brain Sciences 15:75-91. [DTK]

Kenrick, D. T., Nieuweboer, S. \& Buunk, A. P. (2010) Universal mechanisms and cultural diversity: Replacing the blank slate with a coloring book. In: Evolution, culture, and the human mind, ed. M. Schaller, A. Norenzayan, S. Heine, T. Yamagishi \& T. Kameda, pp. 257-72. Psychology Press. [DTK]

Kenrick, D. T. \& Sheets, V. (1993) Homicidal fantasies. Ethology and Sociobiology 14:231-46. [rJA, DTK]
Kilgallon, S. J. \& Simmons, L. W. (2005) Image content influences men’s semen quality. Biology Letters 1:253-55. [FK]

Kim, K. \& Cho, Y. (1992) Epidemiological survey of spousal abuse in Korea. In: Intimate violence: Interdisciplinary perspectives, ed. E. C. Viano, pp. 277-82. Hemisphere. [aJA]

Kimura, D. (1999) Sex and cognition. MIT Press. [NP]

Kirkpatrick, L. A. \& Epstein, S. (1992) Cognitive-experiential self-theory and subjective probability: Further evidence for two conceptual systems. Journal of Personality and Social Psychology 63:534-44. [aJA]

Kirkpatrick, M. \& Ryan M. (1991) The evolution of mating preferences and the paradox of the lek. Nature 350:33-38. [CB]

Knauft, B. M. (1991) Violence and sociality in human evolution. Current Anthropology 32:391-428. [aJA]

Knickmeyer, R., Baron-Cohen, S., Raggatt, P., Taylor, K. \& Hackett, G. (2005) Foetal testosterone and empathy. Hormones and Behavior 49:282-92. [TED]

Knight, G. P., Fabes, R. A. \& Higgins, D. A. (1996) Concerns about drawing causal inferences from meta-analyses: An example in the study of gender differences in aggression. Psychological Bulletin 119:410-21. [aJA]

Knight, G. P., Guthrie, I. K., Page, M. C. \& Fabes, R. A. (2002) Emotional arousal and gender differences in aggression: A meta-analysis. Aggressive Behavior 28:366-93. [aJA]

Kokko, K. \& Pulkkinen, L. (2000) Aggression in childhood and long-term unemployment in adulthood: A cycle of maladaptation and some protective factors. Developmental Psychology 36:463-72. [RET]

Kou, R., Chou, S-Y., Huang, Z. Y. \& Yang, R-L. (2008) Juvenile hormone levels are increased in winners of cockroach fights. Hormones and Behavior $54: 521-27 . \quad[\mathrm{rJA}]$

Kruger, D. J. \& Nesse, R. M. (2006) An evolutionary life-history framework for understanding sex differences in human mortality. Human Nature 17:74-97. [aJA]

Kumagai, F. \& Straus, M. A. (1983) Conflict resolution tactics in Japan, India, and the USA. Journal of Comparative Family Studies 14:377-92. [aJA]

Kyle, U. G., Genton, L., Lukaski, H. C., Dupertuis, Y. M., Slosman, D. O., Hans, D. \& Pichard, C. (2005) Comparison of fat-free mass and body fat in Swiss and American adults. Nutrition 21:161-69. [aJA]

Lagerspetz, K. M. J., Björkqvist, K. \& Peltonen, T. (1988) Is indirect aggression typical of females? Gender differences in 11- to 12- year-old children. Aggressive Behavior 4:403-14. [arJA]

Lalumière, M. L., Harris, G. T., Quinsey, V. L. \& Rice, M. E. (2005) The causes of rape: Understanding individual differences in male propensity for sexual aggression. APA Press. $[\mathrm{FK}]$

Lancaster, J. B. \& Lancaster, C. S. (1983) Parental investment: The hominid adaptation. In: How humans adapt: A biocultural odyssey, ed. D. Ortner, pp. 33-66. Smithsonian Institution Press. [JFB]

Laroche, D. (2005) Aspects of the context and consequences of domestic violence - Situational couple violence and intimate terrorism in Canada in 1999. Government of Quebec. [rJA]

Lassek, W. D. \& Gaulin, S. J. C. (2008) Waist-hip ratio and cognitive ability: Is gluteofemoral fat a privileged store of neurodevelopmental resources? Evolution and Human Behavior 29:26-34. [AC]

(in press) Costs and benefits of fat-free muscle mass in men: Relationship to mating success, dietary requirements, and natural immunity. Evolution and Human Behavior. [SJCG, AS]

Laub, J. H., Nagin, D. S. \& Sampson, R. J. (1998) Trajectories of change in criminal offending: Good marriages and the desistance process. American Sociological Review 63:225-38. [rJA]

Leaper, C. \& Friedman, C. K. (2007) The socialization of gender. In: Handbook of socialization: Theory and research, ed. J. E. Grusec \& P. D. Hastings, pp. 561-87. Guilford Press. [aJA]

LeBlanc, S. \& Register, K. E. (2003) Constant battles: The myth of the peaceful, noble savage. St. Martin's Press. [JFB, DDPJ]

Leigh, S. R. (1992) Patterns of variation in the ontogeny of primate body size dimorphism. Journal of Human Evolution 23:27-50. [SJCG]

Leigh, S. R. \& Shea, B. T. (1995) Ontogeny and evolution of adult body size dimorphism in apes. American Journal of Primatology 36:37-60. [SJCG]

Leonard, J. (2005) Bateman's principle and simultaneous hermaphrodites: A paradox. Integrative and Comparative Biology 45:856-73. [CB]

Leunissen, J. \& van Vugt, M. (unpublished) I love the man in the uniform: Female attraction to male warriors. Unpublished manuscript: University of Kent. [DDPJ]

Leutenegger, W. (1982) Scaling of sexual dimorphism in body weight and canine size in primates. Folia Primatologica 37(3-4):163-76. [JAS]

Levinson, D. (1989) Family violence in cross-cultural perspective. Sage. [aJA]

Levitt, S. D. (2004) Understanding why crime fell in the 1990s: Four factors that explain the decline and six that do not. Journal of Economic Perspectives 18:163-90. [aJA]

Lewis, M., Alessandri, S. \& Sullivan, M. (1990) Violation of expectancy, loss of control, and anger expressions in young infants. Developmental Psychology 26:745-51. [CB] 
Lieberman, D. (2006) Causal explanations of human behavior: From culture to psychology or from psychology to culture? Psychological Inquiry 17:75-95. [rJA]

Lightdale, J. R. \& Prentice, D. A. (1994) Rethinking sex differences in aggression: Aggressive behavior in the absence of social roles. Personality and Social Psychology Bulletin 20:34-44. [aJA, AHE]

Lindenfors, P., Gittleman, J. L. \& Jones, K. E. (2007) Sexual size dimorphism in mammals. In: Sex, size, and gender roles: Evolutionary studies of sexual size dimorphism, ed. D. J. Fairbairn, W. U. Blanckenhorn \& T. Székely, pp. 16-26. Oxford University Press. [aJA]

Lindle, R. S., Metter, E. J., Lynch, N. A., Fleg, J. L., Fozard, J. L., Tobin, J., Roy, T. A. \& Hurley, B. F. (1997) Age and gender comparisons of muscle strength in 654 women and men aged 20-93. Journal of Applied Physiology 83:1581-87. [aJA]

Lippa, R. A. (in press) Sex differences in personality traits and gender-related occupational preferences across 53 nations: Testing evolutionary and socialenvironmental theories. Archives of Sexual Behavior. [aJA]

Loeber, R. \& Hay, D. (1997) Key issues in the development of aggression and violence from childhood to early adulthood. Annual Review of Psychology 48:371-410. [JMB]

Loeber, R., Lacourse, E. \& Homish, D. L. (2005) Homicide, violence, and developmental trajectories. In: Developmental origins of aggression, ed. R. E. Tremblay, W. W. Hartup \& J. Archer, pp. 202-19. Guilford Press. [RET]

Lorenz, K. (1971) Studies in animal and human behavior, vol. II, trans R. Martin Methuen. [rJA]

Low, B. S. (1988) Pathogen stress and polygyny in humans. In: Human reproductive behavior, ed L. Betzig, M. Borgerhoff Mulder \& P. Turke, pp. 115-28. Cambridge University Press. [aJA]

(1989) Cross-cultural patterns in the training of children: An evolutionary perspective. Journal of Comparative Psychology 103:311-19. [rJA]

(1990) Marriage systems and pathogen stress in humans. American Zoologist 30:325-39. [arJA]

Luk, K. D. K., Lu, W. W., Kwan, W. W., Hu, Y., Wong, Y. W., Law, K. K. P. \& Leong, J. A. (2003) Isokinetic and isometric muscle strength of Chinese young males in Taiwan. Ergonomics 40:576-90. [aJA]

Lumsden, C. J. \& Wilson, E. O. (1981) Genes, mind, and culture: The coevolutionary process. Harvard University Press. [PLvdB]

Lynn, J. A. (2008) Women, armies, and warfare in early modern Europe. Cambridge University Press. [PLvdB]

Maccoby, E. E. (1988) Gender as a social category. Developmental Psychology 24:755-65. [arJA, JFB]

(1998) The two sexes: Growing up apart, coming together. Belknap Press of Harvard University Press. [aJA]

Maccoby, E. E. \& Jacklin, C. N. (1974) The psychology of sex differences. Stanford University Press. [aJA]

Mager, J. N., Walcott, C. \& Piper, W. H. (2007) Male common loons, Gavia immer, communicate body mass and condition through dominant frequencies of territorial yodels. Animal Behaviour 73:683-90. [aJA]

Markovits, H., Benenson, J. F. \& Dolenszky, E. (2001) Evidence that children and adolescents have internal models of peer interactions that are gender differentiated. Child Development 72:879-86. [JFB]

Markovits, H., Benenson, J. F. \& White, S. (2006) Gender and gender priming: Differences in speed of processing of information relating to dyadic and group contexts. Journal of Experimental Social Psychology 42:662-67. [JFB]

Marlowe, F. (2004) Marital residence among foragers. Current Anthropology 45:277-88. [aJA]

Maynard Smith, J. (1982) Evolution and the theory of games. Cambridge University Press. [aJA]

Maynard Smith, J. \& Price, G. (1973) The logic of animal conflict. Nature 246(5427):15. [AS]

McCrae, R. R. \& Costa, P. T. (1990) Personality in adulthood. Guilford. [rJA]

McDermott, R. \& Cowden, J. (2001) The effects of uncertainty and sex in a simulated crisis game. International Interactions 27:353-80. [DDPJ]

McElligott, A. G., O’Neill, K. P. \& Hayden, T. J. (1999) Cumulative long-term investment in vocalization and mating success of fallow bucks, Dama dama. Animal Behaviour 57:1159-67. [aJA]

McGowan, P. O., Sasaki, A., D’Alessio, A. C., Dymov, S., Labonté, B., Szyf, M Turecki, G. \& Meaney, M. J. (2009) Epigenetic regulation of the glucocorticoid receptor in human brain associates with childhood abuse. Nature Neuroscience 12(3):342-48. [RET]

McGrew, W. C. (1972) An ethological study of children's behavior. Academic Press. [aJA]

McHenry, H. M. \& Coffing, K. (2000) Australopithecus to Homo: Transformations in body and mind. Annual Review of Anthropology 29:125-46. [aJA]

McKinney, F., Cheng, K. M. \& Bruggers, D. J. (1984) Sperm competition in apparently monogamous birds. In: Sperm competition and evolution of animal mating systems, ed. R. L. Smith, pp. 523-45. Academic Press. [FK]

McKinney, F., Derrickson, S. R. \& Mineau, P. (1983) Forced copulation in waterfowl. Behavior 86:250-94. [FK]
McKinney, F. \& Stolen, P. (1982) Extra-pair-bond courtship and forced copulation among captive green-winged teal (Anas crecca carolinensis). Animal Behaviour 30:461-74. [FK]

McNaughton, N. \& Corr, P. J. (2004) A two-dimensional neuropsychology of defense: Fear/anxiety and defensive distance. Neuroscience and Biobehavioral Reviews 28:285-305. [PJC]

(2008) The neuropsychology of fear and anxiety: A foundation for Reinforcement Sensitivity Theory. In: The reinforcement sensitivity theory of personality, ed. P. J. Corr, pp. 44-94. Cambridge University Press. [PJC]

McNeill, W. H. (1995) Keeping together in time: Dance and drill in human history. Harvard University Press. [DDPJ]

Meaney, M. (2001) Maternal care, gene expression, and the transmission of individual differences in stress reactivity across generations. Annual Review of Neuroscience 24:1161-92. [RET]

Meltzer, H., Gatward, R., Goodman, R. \& Ford, T. (2000) The mental health of children and adolescents in Great Britain. Office for National Statistics. [TED]

Meo, N. (2006) Women who kill with guns and blades. The Times (London) April 1, p. $46 . \quad[\mathrm{KRB}]$

Miller, G. F. (2000) Mental traits as fitness indicators: Expanding evolutionary psychology's adaptationism. In: Evolutionary approaches to human reproductive behavior, ed. D. LeCroy \& P. Moller. Annals of the New York Academy of Science 709:62-74. [JAS]

(2001) Aesthetic fitness: How sexual selection shaped artistic virtuosity as a fitness indicator and aesthetic preferences as mate choice criteria. Bulletin of Psychology and the Arts (Special issue on Evolution, Creativity, and Aesthetics) 2(1):20-25. [JAS]

(2006) Debating sexual selection and mating strategies. Science 312:693. [CB]

Mirrlees-Black, C., Budd, T., Partridge, S. \& Mayhew, P. (1998) The 1998 British Crime Survey. Government Statistical Service (Home Office, London). [arJA]

Moffit, T. E., Caspi, A., Krueger, R. F., Magdol, L., Margolin, G., Silva, P. A. \& Sydney, R. (1997) Do partners agree about abuse in their relationahip? A psychometric evaluation of interpartner aggression. Psychological Assessment 9:47-56. [r]A]

Moore, S. L. \& Wilson, K. (2002) Parasites as a viability cost of sexual selection in natural populations of mammals. Science 297:2015-18. [SJCG]

Mullen, P. E. \& Martin, J. (1994) Jealousy: A community study. British Journal of Psychiatry 164:35-43. [rJA]

Muller, M. N., Kahlenberg, S. M., Thompson, M. E. \& Wrangham, R. W. (2007) Male coercion and the costs of promiscuous mating for female chimpanzees. Proceedings of the Royal Society of London B 274:1009-24. [aJA]

Murdock, G. P. (1967) Ethnographic atlas. University of Pittsburgh Press. [aJA]

Muscarella, F. \& Cunningham, M. R. (1996) The evolutionary significance and social perception of male pattern baldness and facial hair. Ethology and Sociobiology 17:99-117. [aJA]

Nagin, D. S. \& Tremblay, R. E. (1999) Trajectories of boys' physical aggression, opposition, and hyperactivity on the path to physically violent and nonviolent juvenile delinquency. Child Development 70(5):1181-96. [RET]

Naimark, N. M. (1995) The Russians in Germany: A history of the Soviet zone of occupation, 1945-1949. Belknap Press of Harvard University Press. [DDPJ]

Nannini, D. K. \& Meyers, L. S. (2000) Jealousy in sexual and emotional infidelity: An alternative to the evolutionary explanation. Journal of Sex Research 37:117-22. [rJA]

National Institute of Child Health and Development (2004) Trajectories of physical aggression from toddlerhood to middle school. Monographs of the Society for Research in Child Development, Serial No. 278, pp. 1-128. [RET]

Navarrete, C. D., Fessler, D. M. T., Fleischman, D. S. \& Geyer, J. (2009) Race bias tracks conception risk across the menstrual cycle. Psychological Science 20(6):661-65. [DDPJ]

Nisbett, R. E. \& Cohen, D. (1996) Culture of honor: The psychology of violence in the South. Westview Press. [arJA]

Oberst, U., Charles, C. \& Chamarro, A. (2005) Influence of gender and age in aggressive dream content of Spanish children and adolescents. Dreaming $15: 170-77 . \quad$ [MS ]

Öhman, A. \& Mineka, S. (2001) Fears, phobias, and preparedness: Toward an evolved module of fear and fear learning. Psychological Review 108:483-522. [DHB]

Oliver, M. B. \& Hyde, J. S. (1993) Gender diffenences in sexuality: A meta-analysis. Psychological Bulletin 114:29-51. [MS]

Ostrov, J. \& Keating, C. (2004) Gender differences in preschool aggression during free play and structured interactions: An observational study. Social Development 13:255-77. [CB]

Owens, I. P. F. (2002) Sex differences in mortality rates. Science 297:2008-2009. [SJCG]

Pajer, K., Tabbah, R., Gardner, W., Rubin, R. T., Czambel, R. K. \& Wang, Y. (2006) Adrenal androgen and gonadal hormone levels in adolescent girls with conduct disorder. Psychoneuroendocrinology 31:1245-56. [TED] 
Panksepp, J. (1971) Aggression elicited by electrical stimulation of the hypothalamus in albino rats. Physiology and Behavior 6:321-29. [PJC]

Parens, H. (2008) The development of aggression in early childhood. Jason Aronson. [CB]

Parent, C. I. \& Meaney, M. J. (2008) The influence of natural variations in maternal care on play fighting in the rat. Developmental Psychobiology 50(8):767-76. [RET]

Parker, G. A. (1970) Sperm competition and its evolutionary consequences in the insects. Biological Reviews 45:525-67. [FK]

(1974a) Assessment strategy and the evolution of fighting behavior. Journal of Theoretical Biology 47:223-43. [aJA]

(1974b) Courtship persistence and female-guarding as male time investment strategies. Behaviour 48:157-84. [rJA]

Parker, G. A., Baker, R. R. \& Smith, V. G. (1972) The origin and evolution of gamete dimorphism and the male-female phenomenon. Journal of Theoretical Biology 36(3):529-53. [AS]

Pasterski, V., Hindmarsh, P., Geffner, M., Brook, C., Brain, S. \& Hines, M. (2007) Increased aggression and activity level in 3- to 11-year-old girls with congenital adrenal hyperplasia (CAH). Hormones and Behavior 52:368-74. [aJA, TED]

Paus, T., Keshavan, M. \& Giedd, J. N. (2008) Why do many psychiatric disorders emerge during adolescence? Nature Reviews Neuroscience 9(12):947-57. [DT]

Pawlowski, B., Atwal, R. \& Dunbar, R. I. M. (2008) Sex differences in everyday risktaking behavior in humans. Evolutionary Psychology 6:29-42. [aJA]

Pawlowski, B. \& Grabarczyk, M. (2003) Center of body mass and the evolution of

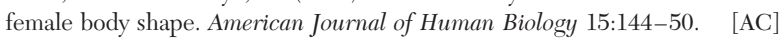

Pawlowski, B. \& Koziel, S. (2002) The impact of traits offered in personal advertisements on response rates. Evolution and Human Behavior 23:139-49. [BK]

Pellegrini, A. D. (2004) Sexual segregation in childhood: A review of evidence for two hypotheses. Animal Behaviour 68:435-43. [aJA, ADP]

Pellegrini, A. D. \& Long, J. D. (2003) A sexual selection theory longitudinal analysis of sexual segregation and integration in early adolescence. Journal of Experimental Child Psychology 85:257-78. [ADP]

Pellegrini, A. D., Long, J. D., Roseth, C., Bohn, K. \& Van Ryzin, M. (2007a) A short-term longitudinal study of preschool children's sex segregation: The role of physical activity, sex, and time. Journal of Comparative Psychology 121:282-89. [ADP]

Pellegrini, A. D., Roseth, C., Mliner, S. C. Bohn, Van Ryzin, M., Vance, N., Cheatham, C. L. \& Tarullo, A. (2007b) Social dominance in preschool classrooms. Journal of Comparative Psychology 121:54-64. [ADP]

Pellegrini, A. D. \& Smith, P. K. (1998) Physical activity play: The nature and function of a neglected aspect of play. Child Development 69:577-98. [ADP]

Peper, J. S., Brouwer, R. M., Schnack, H. G., Van Baal, G. C., Van Leeuwen, M., Van den Berg, S. M., Delemarre-Van de Waal, H. A., Boomsma, D. I., Kahn, R. S. \& Hulshoff Pol, H. E. (2009) Sex steroids and brain structure in pubertal boys and girls. Psychoneuroendocrinology 34(3):332-42. [DT]

Perkins, A. M. \& Corr, P. J. (2006) Reactions to threat and personality: Psychometric differentiation of intensity and direction dimensions of human defensive behavior. Behavioural Brain Research 169:21-28. [PJC]

Perry, D. G. \& Bussey, K. (1979) The social learning theory of sex differences: Imitation is alive and well. Journal of Personality and Social Psychology 37:1699-712. [aJA]

Perry, D. G., Perry, L. C. \& Weiss, R. J. (1989) Sex differences in the consequences that children anticipate for aggression. Developmental Psychology 25:312-19. [aJA]

Petralia, S. M. \& Gallup, G. (2002) Effects of a sexual assault scenario on handgrip strength across the menstrual cycle. Evolution and Human Behavior 23:3-10. [JAS]

Pheasant, S. T. (1983) Sex differences in strength - some observations on their variability. Applied Ergonomics 14:205-11. [aJA]

Platt, J. R. (1964) Strong inference. Science 146:347-53. [AJF]

Plavcan, J. M. (2000) Inferring social behavior from sexual dimorphism in the fossil record. Journal of Human Evolution 39:327-44. [aJA, AHE]

Plavcan, J. M. \& van Schaik, C. P. (1997a) Interpreting hominid behavior on the basis of sexual dimorphism. Journal of Human Evolution 32:345-74. [aJA, AHE]

(1997b) Intrasexual competition and body weight dimorphism in anthropoid primates. American Journal of Physical Anthropology 103:37-68. [aJA, JAS]

(2005) Canine dimorphism. Evolutionary Anthropology: Issues, News, and Reviews 2:208-14. [AHE]

Plomin, R., DeFries, J. C., McClearn, G. E. \& McGuffin, P. (2001) Behavioral genetics, 4th edition. Worth. [PJC]

Pomiankowski, A. \& Møller, A. P. (1995) A reolution to the lek paradox. Proceedings of the Royal Society of London B 160:21-29. [aJA]

Popma, A., Vermeiren, R., Geluk, C. A. M. L., Rinne, T., Van den Brink, W., Knol, K. L., Jansen, L. M., Van Engeland, H. \& Doreleijers, T. A. (2007) Cortisol moderates the relationship between testosterone and aggression in delinquent male adolescents Biological Psychiatry 61:405-411. [DT]

Potts, M. \& Hayden, T. (2008) Sex and war: How biology explains warfare and terrorism and offers a path to a safer world. Benbella Books. [DDPJ]

Pound, N. (2002) Male interest in visual cues of sperm competition risk. Evolution and Human Behavior 23:443-66. [FK]

Promislow, D. E. L. (1992) Costs of sexual selection in natural populations of mammals. Proceedings of the Royal Society of London B 247:203-10. [SJCG]

Promislow, D. E. L., Montgomerie, R. \& Martin, T. E. (1992) Mortality costs of sexual dimorphism in birds. Proceedings of the Royal Society of London B 250:143-50. [SJCG]

Putman, P., Hermans, E. J. \& van Honk, J. (2004) Emotional Stroop performance for masked angry faces: It's BAS, not BIS. Emotion 4:305-11. [aJA]

Puts, D. A., Gaulin, S. J. C. \& Verdolini, K. (2006) Dominance and the evolution of sexual dimorphism in human voice pitch. Evolution and Human Behavior 27:283-96. [aJA]

Puts, D. A., Hodges, C. R., Cárdenas, R. A. \& Gaulin, S. J. C. (2007) Men’s voices as dominance signals: Vocal fundamental and formant frequencies influence dominance attributions among men. Evolution and Human Behavior 28:340-44. [aJA]

Quetelet, A. (1833/1984) Recherches sur le penchant au crime aux différens âges. M. Hayez. (English edition, 1984: Research on the propensity for crime at different ages, trans. S. F. Sylvester. Anderson). [aJA]

Raine, A., Brennan, P. \& Farrington, D. P. (1997) Biosocial bases of violence: Conceptual and theoretical issues. In: Biosocial bases of violence, ed. A. Raine, P. Brennan, D. P. Farrington \& A. A. Mednick, pp. 1-20. Plenum Press. [aJA]

Raine, A., Dodge, K., Loeber, R., Gatzke-Kopp, L., Lynam, D., Reynolds, C., Stouthamer-Loeber, M. \& Liu, J. (2006) The Reactive-Proactive Aggression Questionnaire: Differential correlates of reactive and proactive aggression in adolescent boys. Aggressive Behavior 32:159-71. [rJA]

Ralls, K. (1976) Mammals in which females are larger than the males. Quarterly Review of Biology 51:245-76. [aJA]

Reed, C. L. (2005) War stress heavier on women. Chicago Sun-Times, May 8, p. $4 . \quad[\mathrm{KRB}]$

Reiner, W. G. \& Gearhart, J. P. (2004) Disconcordant sexual identity in some genetic males with cloacal extrophy assigned to female sex at birth. The New England Journal of Medicine 350:333-41. [DHB]

Rhee, S. H. \& Waldman, I. D. (2002) Genetic and environmental influences on antisocial behavior: A meta-analysis of twin and adoption studies. Psychological Bulletin 128:490-529. [BK]

Richardson, D. R. \& Green, L. R. (1999) Social sanction and threat explanations of gender effects on direct and indirect aggression. Aggressive Behavior 25:425-34. [aJA]

Richerson, P. J. \& Boyd, R. (2005) Not by genes alone: How culture transformed human evolution. University of Chicago Press. [AHE]

Rielly, R. J. (2000) Confronting the tiger: Small unit cohesion in battle. Military Review 80(6):61-65. [DDPJ]

Rosen, L. N., Wright, K., Marlowe, D., Bartone, P. \& Gifford, R. K. (1999) Gender differences in subjective distress attributable to anticipation of combat among U.S. Army soldiers deployed to the Persian Gulf during Operation Desert Storm. Military Medicine 164:753-57. [KRB]

Rosenthal, D. (1984) Meta-analytic procedures for social research. Sage. [aJA] Roughgarden, J., Oisha, M. \& Akcay, E. (2006) Reproductive social behavior: Cooperative games to replace sexual selection. Science 311:965-69. [CB, $\mathrm{AC}]$

Rubin, L. (1985) Just friends: The role of friendship in our lives. Harper \& Row. $[\mathrm{rJA}]$

Ruff, J. R. (2001) Violence in early modern Europe 1500-1800. Cambridge University Press. [rJA]

Russell, D. E. H. (1982) Rape in marriage. Macmillan. [FK]

Rutter, A. \& Hine, D. W. (2005) Sex differences in workplace aggression: An investigation of moderation and mediation effects. Aggressive Behavior 31:254-70. [arjA]

Salzano, F. M., Neel, J. V. \& Maybury-Lewis, D. (1967) Further studies of the Xavante Indians. I. Demographic data on two additional villages: Genetic structure of the tribe. American Journal of Human Genetics 19:463-89. [aJA]

Savin-Williams, R. C. (1980) Social interactions of adolescent females in natural groups. In: Friendship and social relations in children, ed. H. C. Foot, A. J. Chapman \& J. R. Smith, pp. 343-64. Wiley. [JFB]

Scarpa, A. \& Haden, S. C. (2006) Psychophysiological, behavioral, and emotional distinctions between childhood reactive and proactive aggression. Paper presented at the XVIII World Meeting of the International Society for Research on Aggression, Minneapolis, MN, June 2006. [rJA]

Schaller, M. \& Murray, D. R. (2008) Pathogens, personality and culture: Disease prevalence predicts worldwide variability in sociosexuality, extraversion, and 
openness to experience. Journal of Personality and Social Psychology 95:212-21. [rJA, DTK]

Scharnberg, K. (2005) Stresses of battle hit female GIs hard: VA study hopes to find treatment for disorder. Chicago Tribune March 22, p. C-1. [KRB]

Schmitt, D. P. \& Buss, D. M. (1996) Mate attraction and competitor derogation: Context effects on perceived effectiveness. Journal of Personality and Social Psychology 70:1185-204. [DMB]

Schmitt, D. P., Realo, A., Voracek, M. \& Allik, J. (2008) Why can't a man be more like a woman? Sex differences in Big Five personality traits across 55 cultures. Journal of Personality and Social Psychology 94:168-82. [AHE]

Schredl, M. (2003) Continuity between waking and dreaming: A proposal for a mathematical model. Sleep and Hypnosis 5:38-52. [MS]

Schredl, M., Ciric, P., Bishop, A., Gölitz, E. \& Buschtöns, D. (2003) Content analysis of German students' dreams: Comparison to American findings. Dreaming 13:237-43. [MS]

Schredl, M. \& Keller, K. (2008-2009) Dream content in a representative German sample: Gender differences and the effects of other socio-demographic variables. Imagination, Cognition and Personality 28:37-48. [MS]

Schredl, M. \& Pallmer, R. (1998) Geschlechtsunterschiede in Angstträumen von SchülerInnen (Gender differences in anxiety dreams of school-aged children). Praxis der Kinderpsychologie und Kinderpsychiatrie 47:463-76. [MS]

Schredl, M., Sahin, V. \& Schäfer, G. (1998) Gender differences in dreams: Do they reflect gender differences in waking-life? Personality and Individual Differences 25:433-42. [MS]

Schuster, I. (1983) Women's aggression: An African case study. Aggressive Behavior 9:319-31. [aJA]

(1985) Female aggression and resource scarcity: A cross-cultural perspective. In: The aggressive female, ed. M. Haug, D. Benton, P. F. Brain, B. Olivier \& J. Mos, pp. 185-207. Eden. [aJA]

Sears, R. R., Rau, L. \& Alpert, R. (1965) Identification and child rearing. Stanford University Press. [aJA]

Sefcek, J. A., Brumbach, B. H., Vásquez, G. \& Miller, G. F. (2006) The evolutionary psychology of human mate choice: How ecology, genes, fertility, and fashion influence our mating behavior. In: On the Evolution of Sexual Attraction, ed. M. R. Kauth. Special Issue of the Journal of Psychology and Human Sexuality 18(2/3):125-82. [JAS]

Selander, R. K. (1972) Sexual selection and dimorphism in birds. In: Sexual selection and the descent of man, ed. B. Campbell, pp. 180-230. Aldine. [aJA]

Sell, A. (2005) Regulating welfare tradeoff ratios: Three tests of an evolutionarycomputational model of human anger. Unpublished doctoral dissertation, University of California, Santa Barbara. [aJA]

(2006) Anger face dissected: Why do their faces look like that? Paper presented at the Meeting of the International Society for Research on Aggression World Meeting, Minneapolis, Minnesota, July 2006. [aJA]

Sell, A., Cosmides, L., Tooby, J., Sznycer, D., von Rueden, C. \& Gurven, M. (2009) Human adaptations for the visual assessment of strength and fighting ability from the body and face. Proceedings of the Royal Society of London B 276:575-84. [aJA]

Sell, A., Tooby, J. \& Cosmides, L. (2005) Physical strength predicts entitlement, anger, and attitudes about aggression in men. Paper presented at the Human Behavior and Evolution Society Conference, Austin, Texas, June 2005. [aJA, NP]

Serbin, L. A., Cooperman, J. M., Peters, P. L., Lehoux, P. M., Stack, D. M. \& Schwartzman, A. E. (1998) Intergenerational transfer of psychosocial risk in women with childhood histories of aggression, withdrawal, or aggression and withdrawal. Developmental Psychology 34(6):1246-62. [RET]

Serbin, L. A., O'Leary, K., Kent, R. \& Tonick, I. (1973) A comparison of teacher response to the pre-academic and problem behaviours of boys and girls. Child Development 44:796-804. [CB]

Sergeant, M. J. T., Dickins, T. E., Davies, M. N. O. \& Griffiths, M. D. (2006) Aggression, empathy, and sexual orientation among males. Personality and Individual Differences 40:475-86. [TED]

Shackelford, T. K. \& Buss, D. M. (1997) Marital satisfaction in evolutionary psychological perspective. In: Satisfaction in close relationships, ed. R. J Sternberg \& M. Hojjat, pp. 7-25. Guilford Press. [aJA]

Shackelford, T. K. \& Goetz, A. T. (2007) Adaptation to sperm competition in humans. Current Directions in Psychological Science 16:47-50. [FK]

Shackelford, T. K., Goetz, A. T., Buss, D. M., Euler, H. A. \& Hoier, S. (2005a) When we hurt the ones we love: Predicting violence against women from men's mate retention. Personal Relationships 12:447-63. [rJA, FK]

Shackelford, T. K., LeBlanc, G. J., Weekes-Shackelford, V. A., Bleske-Rechek, A. L., Euler, H. A. \& Hoier, S. (2002) Psychological adaptation to human sperm competition. Evolution and Human Behavior 23:123-38. [FK]

Shackelford, T. K. \& Pound, N., eds. (2006) Sperm competition in humans. Springer. $\quad[\mathrm{FK}]$

Shackelford, T. K., Pound, N. \& Goetz, A. T. (2005b) Psychological and physiological adaptations to sperm competition in humans. Review of General Psychology 9:228-48. [FK]
Sharpe, J. P. \& Desai, S. (2001) The revised neo personality inventory and the MMPI-2 psychopathology five in the prediction of aggression. Personality and Individual Differences 31:505-18. [aJA]

Shettles, L. B. (1961) Conception and birth sex ratios. Obstetrics and Gynecology $18: 122-30 . \quad$ [aJA]

Skarðhamar, T. \& Lyngstad, T. H. (2009) Family formation, fatherhood and crime: An invitation to a broader perspective on crime and family transitions. (Discussion Papers No. 579). Statistics Norway Research Department. [rJA]

Slotter, E. B. \& Finkel, E. J. (in press) $\mathrm{I}^{3}$ Theory: Instigating, impelling, and inhibiting factors in aggression. In: Understanding and reducing aggression, violence, and their consequences, ed. M. Mikulincer \& P. R. Shaver. American Psychological Association. [EJF]

Smith, D. L. (2007) The most dangerous animal. St. Martin's Press. [DMB]

Smith, R. L. (1984) Human sperm competition. In: Sperm competition and the evolution of animal mating systems, ed. R. L. Smith, pp. 601-660. Academic Press. [FK]

Smuts, B. B. (1987a) Gender, aggression, and influence. In: Primate societies, ed. B. B. Smuts, D. L. Cheney, R. M. Seyfarth, R. W. Wrangham \& T. T. Struhsaker, pp. 400-12. University of Chicago Press. [aJA, JFB]

(1987b) Sexual competition and mate choice. In: Primate societies, ed. B. B. Smuts, D. L. Cheney, R. M. Seyfarth, R. W. Wrangham \& T. T. Struhsaker, pp. 385-99. University of Chicago Press. [aJA]

(1992) Male aggression against women: An evolutionary perspective. Human Nature 3:1-44. [aJA]

(1995) The evolutionary origins of patriarchy. Human Nature 6:1-32. [aJA]

Smuts, B., Cheney, D., Seyfarth, R., Wrangham, R. \& Struhsaker, T. (1987) Primate societies. The University of Chicago Press. [AS]

Sroufe, L. (1995) Emotional development. The organization of emotional life in the early years. Cambridge University Press. [CB]

Stamos, D. (2008) Evolution and the big questions: Sex, race, religion, and other matters. Blackwell. [CB]

Starratt, V. G., Goetz, A. T., Shackelford, T. K. \& Stewart-Williams, S. (2008) Men's partner-directed insults and sexual coercion in intimate relationships. Journal of Family Violence 23:315-23. [FK]

Stenberg, C. \& Campos, J. (1990) The development of anger expressions in infancy. In: Psychological and biological approaches to emotions, ed. N. Stein, B. Leventhal \& T. Trabasso, pp. 247-82. Erlbaum. [CB]

Stenberg, C., Campos, J. \& Emde, R. N. (1983) The facial expression of anger in seven-month-old infants. Child Development 54:178-84. [CB]

Stevenson, J., Richman, N. \& Graham, P. (1985) Behaviour problems and language abilities at three years and behavioral deviance at eight years. Journal of Child Psychology and Psychiatry 26:215-30. [CB]

Straus, M. A. (1979) Measuring intrafamily conflict and violence: The Conflict Tactics (CT) Scales. Journal of Marriage and the Family 41:75-88. [aJA]

(1990) The Conflict Tactics Scales and its critics: An evaluation and new data on validity and reliability. In: Physical violence in American families: Risk factors and adaptations to violence in 8,145 families, ed. M. A. Straus \& R. J. Gelles, pp. 49-73. Transaction Publications. [rJA]

(1999) The controversy over domestic violence by women: A methodological, theoretical, and sociology of science analysis. In: Violence in intimate relationships, ed. X.B. Arriaga \& S. Oskamp, pp. 17-44. Sage. [arJA]

(2004) Cross-cultural reliability and validity of the Revised Conflict Tactics Scales: A study of university student dating couples in 17 nations. Cross Cultural Research 38:407-32. [rJA]

Straus, M. A. \& Gelles, R. J. (1988) How violent are American families? Estimates from the National Family Violence Resurvey and other studies In: Family abuse and its consequences: New directions in research, ed. G. T. Hotaling, D. Finkelhor, J. T. Kirkpatrick \& M. A. Straus, pp. 14-36. Sage. [aJA]

Suomi, S. J. (2005) Genetic and environmental factors influencing the expression of impulsive aggression and serotinergic functioning in rhesus monkeys. In: Developmental origins of aggression, ed. R. Tremblay, W. Hartup \& J. Archer, pp. 63-82. Guilford Press. [rJA, RET]

Symons, D. (1978) Play and aggression. Columbia University Press. [AS]

Tanner, J. M. (1970) Physical growth. In: Carmichael's manual of child psychology, vol. 1, ed. P. H. Mussen, pp. 77-155. Wiley. [aJA]

(1989) Foetus into man: Physical growth from conception to maturity, 2nd edition. Castlemead. [aJA]

Tedeschi, J. T. \& Felson, R. B. (1994) Violence, aggression, and coercive actions. American Psychological Association. [arjA]

Thanzami, V. L. \& Archer, J. (2005) Beliefs about aggression in British students from individualistic and collectivist cultures. Aggressive Behavior 31:350-58. [aJA]

Thayer, B. A. (2004) Darwin and international relations: On the evolutionary origins of war and ethnic conflict. University Press of Kentucky. [DDPJ]

Thornhill, R., Fincher, C. L. \& Aran, D. (2009) Parasites, democratization, and the liberalization of values across contemporary countries. Biological Reviews 84:113-31. [rJA] 
Thornhill, R. \& Thornhill, N. W. (1992) The evolutionary psychology of men's coercive sexuality. Behavioral and Brain Sciences 15:363-421. [FK]

Tieger, T. (1980) On the biological basis of sex differences. Child Development 51:943-63. [aJA]

Tinbergen, N. (1963) On the aims and methods of ethology. Zeitschrift für Tierpsychologie 20:410-433. [rJA]

Tolan, P., Gorman-Smith, D. \& Henry, D. (2006) Family violence. Annual Review of Psychology 57(1):557-83. [JMB]

Tooby, J. (1982) Pathogens, polymorphism and the evolution of sex. Journal of Theoretical Biology 97:557-76. [AS]

Tooby, J. \& Cosmides, L. (1988) The evolution of war and its cognitive foundations Institute for Evolutionary Studies, Technical Report No. 88-1. [DMB]

Tovee, M. J. \& Cornelissen, P. L. (1999) The mystery of female beauty. Nature 399(6733):215-16. [AC]

Tremblay, R. E. (2008) Understanding development and prevention of chronic physical aggression: Towards experimental epigenetic studies. Philosophical Transactions of the Royal Society of London 363(1503):2613-22. [RET]

Tremblay, R. E., Japel, C., Pérusse, D., McDuff, P., Boivin, M., Zoccolillo, M. \& Montplaisir, J. (1999) The search for the age of "onset" of physical aggression: Rousseau and Bandura revisted. Criminal Behavior and Mental Health 9(1):8-23. [arJA, CB, RET]

Tremblay, R. E., Nagin, D., Seguin, J., Zoccolillo, M., Zelazo, P., Boivin, M., Perusse, D. \& Japel, C. (2004) Physical aggression during early childhood: Trajectories and predictors. Pediatrics 114:43-50. [CB]

Tremblay, R. E., Schaal, B., Boulerice, B., Arseneault, L., Soussignan, R. G., Paquette, D. \& Laurent, D. (1998) Testosterone, physical aggression, dominance, and physical development in early adolescence. International Journal of Behavioral Development 22:753-57. [aJA]

Trivers, R. (1972) Parental investment and sexual selection. In: Sexual selection and the descent of man, ed. B. B. Campbell, pp. 136-79. Aldine. [arJA, SJCG]

Twenge, J. M. (1997a) Attitudes toward women, 1970-1995. Psychology of Women Quarterly 21:35-51. [aJA]

(1997b) Changes in masculine and feminine traits over time: A meta-analysis. Sex Roles 36:305-25. [aJA]

(2001) Changes in women's assertiveness in response to status and roles: A crosstemporal meta-analysis, 1931-1993. Journal of Personality and Social Psychology 81:133-45. [aJA]

Tyson, A. S. (2005) For female GIs, combat is a fact: Many duties in Iraq put women at risk despite restrictive policy. Washington Post May 13, p. A-1. [KRB]

United Nations Development Programme Human Development Report (1997) Oxford University Press. [aJA]

United Nations Development Programme Human Development Report (2005) Available at: http://hdr.undp.org/reports/global/2005/ [aJA]

Vaillancourt, T. \& Sharma, A. (2008) Undergraduate females' use of indirect aggression: Under the right circumstances everyone aggresses. Paper presented at the XVIII World Meeting of the International Society for Research on Aggression, Budapest, Hungary, July 2008. [r]A]

Valera, F., Hoi, H. \& Kristin, A. (2003) Male shrikes punish unfaithful females. Behavioral Ecology 14:403-408. [BK, FK]

van der Dennen, J. M. G. (1995) The origin of war. Rigin. [DMB]

van Honk, J. (2009) Testosterone and human social behavior. Paper presented by invitation at the Association for Psychological Science, San Francisco, May 2009. [DT]

van Honk, J., Tuiten, A., Hermans, E. J., Putman, P., Koppeschaar, H. P., Thijssen, J., Verbaten, R. \& van Doornen, L. (2001) A single administration of testosterone induces cardiac acceleration response to angry faces in healthy young women. Psychoneuroendocrinology 29:937-43. [aJA]

van Hooff, J. A. R. A. M. (1972) A comparative approach to the phylogyny of laughter and smiling. In: Non-verbal communication, ed. R. A. Hinde, pp. 209-41. Cambridge University Press. [rJA]

van Vugt, M., De Cremer, D. \& Janssen, D. (2007) Gender differences in cooperation and competition: The male warrior hypothesis. Psychological Science 18:19-23. [arJA, DDPJ]

Vitaro, F. \& Brendgen, M. (2005) Proactive and reactive aggression. In: Developmental origins of aggression, ed. R. Tremblay, W. W. Hartup \& J. Archer, pp. 178-201. Guilford. [rJA]

Vitaro, F., Brendgen, M., Larose, S. \& Tremblay, R. E. (2005) Kindergarten disruptive behaviors, protective factors, and educational achievement by early adulthood. Journal of Educational Psychology 97(4):617-29. [RET]

Waddington, C. H. (1957) The strategy of genes. Allen and Unwin. [aJA]

Wade, M. \& Shuster, S. (2002) The evolution of parental care in the context of sexual selection: A critical reassessment of parental investment theory. American Naturalist 160:285-92. [CB]

Walby, S. \& Allen, J. (2004) Domestic violence, sexual assault and stalking: Findings from the British Crime Survey. Home Office Research, Development and Statistical Directorate, London. [r]A]

Walker, L. E. (1979) The battered woman. Harper \& Row. [FK]
(1989) Psychology and violence against women. American Psychologist 44:659-702. [aJA]

Wallace, A. (1871) Review of The Descent of Man and Selection in Relation to Sex by Charles Darwin. Academy 2:177-83. Available at: http://www.wku.edu/ $\sim$ smithch/wallace/S186.htm. [CB]

Waterman, D., de Jong, M. A. \& Magdeliyns, R. (1988) Gender, sex role orientation and dream content (Sleep '86). Gustav Fischer. [MS]

Watson, J. B. (1919) Psychology from the standpoint of a behaviorist. Lippincott. [PJC] Webb, I. A. (2007) The role of evolutionary and social factors in the same-sex and partner aggression. Unpublished Master's dissertation, University of Central Lancashire, Preston, United Kingdom. [rJA]

Weisner, T. S. \& Wilson-Mitchell, J. E. (1990) Nonconventional family lifestyles and sex typing in six-year-olds. Child Development 61:1915-33. [DHB]

Weston, E. M., Friday, A. E., Johnstone, R. A. \& Schrenk, F. (2004) Wide faces or large canines? The attractive versus the aggressive primate? Proceedings of the Royal Society of London B (Suppl.) 271(S):416-19. [aJA]

Weston, E. M., Friday, A. E. \& Lio, P. (2007) Biometric evidence that sexual selection has shaped the hominid face. PLOS ONE 2 (8):e710. Available online: doi: 10.1371/journal.pone.0000710). [aJA]

White, G. L. (1981) Some correlates of romantic jealousy. Journal of Personality 49:129-47. [rJA]

Wilder, J. A., Mobasher, Z. \& Hammer, M. F. (2004) Genetic evidence for unequal effective population sizes of human females and males. Molecular Biology and Evolution 21:2047-57. [NP]

Wilkinson, R. \& Pickett, K. (2009) The spirit level: Why more equal societies almost always do better. Allen Lane. [rJA]

Williams, G. (1957) Pleiotropy, natural selection, and the evolution of senescence. Evolution 11(4): 398-411. [AS]

(1966) Adaptation and natural selection. Princeton University Press.

(1975) Sex and evolution. Princeton University Press. [CB]

Wilson, G. \& Rahman, Q. (2005) Born gay: The psychobiology of sexual orientation. Peter Owen. [TED]

Wilson, M. \& Daly, M. (1992) The man who mistook his wife for a chattel. In: The adapted mind, ed. J. H. Barkow, L. Cosmides \& J. Tooby, pp. 289-321. Oxford University Press. [arJA, FK]

(1996) Male sexual proprietariness and violence against wives. Current Directions in Psychological Science 5:2-7. [rJA, EC]

(1997) Life expectancy, economic inequality, homicide, and reproductive timing in Chicago neighborhoods. British Medical Journal 314:1271-74. [TED, NP] (2006) Are juvenile offenders extreme discounters? Psychological Science 17:989-94. [aJA]

Wilson, M., Daly, M. \& Pound, N. (2002) An evolutionary psychological perspective on the modulation of competitive confrontation and risk-taking. In: Hormones, brain and behavior, vol. 5., ed. D. W. Pfaff, A. P. Arnold, A. M. Etgen, S. E. Fahrbach, R. T. Rubin, pp. 381-408. Academic Press. [NP]

(2009) Sex differences and intrasexual variation in competitive confrontation and risk-taking: An evolutionary psychological perspective. In: Hormones, brain and behavior, 2nd edition, ed. D. W. Pfaff, A. P. Arnold, A. M. Etgen, S. E. Fahrbach, R. T. Rubin, pp. 2825-52. Elsevier. [NP]

Wilson, M. L. \& Wrangham, R. W. (2003) Intergroup relations in chimpanzees. Annual Review of Anthropology 32:263-92. [arJA]

Wingfield, J. C., Hegner, R. E., Dufty Jr., A. M. \& Ball, G. F. (1990) The "challenge hypothesis": Theoretical implications for patterns of testosterone secretion, mating systems, and breeding strategies. American Naturalist 136:829-46. [rJA]

Wolfe, L. D. \& Gray, J. P. (1982) Latitude and intersocietal variation of human sexual dimorphism of stature. Human Ecology 10:409-15. [aJA]

Wood, W. \& Eagly, A. H. (2002) A cross-cultural analysis of the behavior of women and men: Implications for the origins of sex differences. Psychological Bulletin 128:699-727. [arJA, DHB, AHE, NP, AS, JAS]

(2007) Social structural origins of sex differences in human mating. In: The evolution of mind: Fundamental questions and controversies, ed. S. W. Gangestad \& J. A. Simpson, pp. 383-90. Guilford Press. [DTK]

(in press) Gender. In: Handbook of social psychology, 5th edition, ed. S. T. Fiske, D. T. Gilbert \& G. Lindzey. Wiley. [AHE]

Wrangham, R. W. (1987) Evolution of social structure. In: Primate societies, ed. B. B. Smuts, D. L. Cheney, R. M. Seyfarth, R. W. Wrangham \& T. T. Struhsaker, pp. 286-98. University of Chicago Press. [JFB] (1999) Evolution of coalitionary killing. Yearbook of Physical Anthropology 42:1-30. [rJA, JFB]

Wrangham, R. W., Jones, J. H., Laden, G., Pilbeam, D. \& Conklin-Brittain, N. (1999) The raw and the stolen: Cooking and the ecology of human origins. Current Anthropology 40:567-77. [AHE]

Wrangham, R. W. \& Peterson, D. (1996) Demonic males: Apes and the origins of human violence. Bloomsbury. [DDPJ]

Wrangham, R. W. \& Wilson, M. L. (2004) Collective violence: Comparisons between youths and chimpanzees. Annals of the New York Academy of Sciences 1036:233-56. [rJA, DDPJ] 


\section{References/Archer: Sex differences in aggression}

Wrangham, R. W., Wilson, M. L. \& Muller, M. N. (2006) Comparative rates of violence in chimpanzees and humans. Primates 47:14-26. [aJA]

Wright, P. H. (1988) Interpreting research on gender differences in friendship: A case for moderation and a plea for caution. Journal of Social and Personal Relationships 5:367-73. [rJA]

Xiao, G., Lei, L., Dempsey, P. G., Lu, B. \& Liang, Y. (2005) Isometric muscle strength and anthropometric characteristics of a Chinese sample. International Journal of Industrial Ergonomics 35:674-79. [aJA]

Yu, D. W. \& Shepard, G. H. (1999) The mystery of female beauty: Reply. Nature 399(6733):216. [AC]

Zerjal, T., Xue, Y., Bertorelle, G., Wells, R. S., Bao, W., Zhu, S., Qamar, R., Ayub, Q., Mohyuddin, A., Fu, S., Li, P., Yuldasheva, N., Ruzibakiev, R., Xu, J., Shu, Q., Du, R., Yang, H., Hurles, M. E., Robinson, E., Gerelsaikhan, T., Dashnyam, B., Mehdi, S. Q. \& Tyler-Smith, C. (2003) The genetic legacy of the Mongols. American Journal of Human Genetics 72:717-21. [Published electronically January 17. Available at: http://www.pubmedcentral.nih.gov/ articlerender.fogi?tool $=$ pmcentrez\&amp;rendertype $=$ abstract\&artid $=$ 1180246.] [DMB]

Zuckerman, M. (1994) Behavioural expressions and biosocial bases of sensation seeking. Cambridge University Press. [aJA]

Zuckerman, M. \& Kuhlman, D. M. (2000) Personality and risk-taking: Common biosocial factors. Journal of Personality 68:999-1029. [aJA]

Zuckerman, M., Kuhlman, D. M., Joireman, J., Teta, P. \& Kraft, M. (1993) A comparison of three structural models for personality: The big three, the big five, and the alternative five. Journal of Personality and Social Psychology 65:757-68. [aJA]

Zuk, M. \& McKean, K. A. (1996) Sex differences in parasite infection: Patterns and processes. International Journal for Parasitology 26:1009-24. [SJCG] 